\title{
THE COMBINATORICS OF $\mathbb{C}^{*}$-FIXED POINTS IN GENERALIZED CALOGERO-MOSER SPACES AND HILBERT SCHEMES
}

\author{
TOMASZ PRZEZDZIECKI
}

\begin{abstract}
In this paper we study the combinatorial consequences of the relationship between rational Cherednik algebras of type $G(l, 1, n)$, cyclic quiver varieties and Hilbert schemes. We classify and explicitly construct $\mathbb{C}^{*}$-fixed points in cyclic quiver varieties and calculate the corresponding characters of tautological bundles. Furthermore, we give a combinatorial description of the bijections between $\mathbb{C}^{*}$-fixed points induced by the Etingof-Ginzburg isomorphism and Nakajima reflection functors. We apply our results to obtain a new proof as well as a generalization of the $q$-hook formula.
\end{abstract}

\section{Contents}

1. Introduction

2. Rational Cherednik algebras

3. Quiver varieties

5

4. Combinatorics

5. $\mathbb{C}^{*}$-fixed points in quiver varieties

6. Degenerate affine Hecke algebras

7. $\mathbb{C}^{*}$-fixed points under the Etingof-Ginzburg isomorphism 21

8. The higher level $q$-hook formula

9. $\mathbb{C}^{*}$-fixed points under reflection functors 29

10. Connection to the Hilbert scheme 34

References

\section{INTRODUCTION}

Rational Cherednik algebras were introduced by Etingof and Ginzburg 12. They depend on a complex reflection group as well as two parameters $\mathbf{h}$ and $t$. In the case of Weyl groups they coincide with certain degenerations of double affine Hecke algebras introduced earlier by Cherednik [5]. When $t=0$, rational Cherednik algebras have large centres and the corresponding affine varieties are known as generalized Calogero-Moser spaces. In this paper we consider cyclotomic rational Cherednik algebras at $t=0$, i.e., the rational Cherednik algebras $\mathbb{H}_{\mathbf{h}}:=\mathbb{H}_{t=0, \mathbf{h}}\left(\Gamma_{n}\right)$ associated to complex reflection groups $\Gamma_{n}:=(\mathbb{Z} / l \mathbb{Z}) ? S_{n}$ of type $G(l, 1, n)$. The associated generalized Calogero-Moser spaces are especially interesting because they admit a realization as Nakajima quiver varieties. This fact was exploited by Gordon [16, who established a connection between generalized Calogero-Moser spaces and Hilbert schemes of points in the plane.

Let us recall the framework of [16 in more detail. Assume that the variety $\mathcal{Y}_{\mathbf{h}}:=$ Spec $Z\left(\mathbb{H}_{\mathbf{h}}\right)$ is smooth. Etingof and Ginzburg showed in 12 that $\mathcal{Y}_{\mathbf{h}}$ is isomorphic to a cyclic quiver variety $\mathcal{X}_{\theta}(n \delta)$ (see $\$ 3.2$ generalizing Wilson's construction of the Calogero-Moser space in 36. Considering $\mathcal{X}_{\theta}(n \delta)$ as a hyper-Kähler manifold, one can use reflection functors, defined by Nakajima in [30, to construct a hyper-Kähler isometry $\mathcal{X}_{\theta}(n \delta) \rightarrow \mathcal{X}_{-\frac{1}{2}}(\gamma)$ between quiver varieties associated to different parameters. Furthermore, rotation of complex structure yields a diffeomorphism between $\mathcal{X}_{-\frac{1}{2}}(\gamma)$ and a certain GIT quotient $\mathcal{M}_{-\mathbf{1}}(\gamma)$ (see $\$ 3.2$ ). The latter is isomorphic to an irreducible component

2010 Mathematics Subject Classification. 16G99, 05E10.

Key words and phrases. Rational Cherednik algebras, Hilbert schemes, Nakajima quiver varieties, Calogero-Moser space, $q$-hook formula, wreath Macdonald polynomials, degenerate affine Hecke algebras. 
$\mathrm{Hil}_{K}^{\nu}$ of $\mathrm{Hil}_{K}^{\mathbb{Z} / l \mathbb{Z}}$, where $\mathrm{Hil}_{K}$ denotes the Hilbert scheme of $K$ points in $\mathbb{C}^{2}$. The following diagram summarizes all the maps involved:

$$
\mathcal{Y}_{\mathbf{h}} \stackrel{\text { EG }}{\longrightarrow} \mathcal{X}_{\theta}(n \delta) \stackrel{\text { Refl.Fun. }}{\longrightarrow} \mathcal{X}_{-\frac{1}{2}}(\gamma) \stackrel{\text { Rotation }}{\longrightarrow} \mathcal{M}_{-\mathbf{1}}(\gamma) \hookrightarrow \mathrm{Hil}_{K}^{\mathbb{Z} / l \mathbb{Z}} .
$$

Let us explain the parameters. The affine symmetric group $\tilde{S}_{l}$ acts on dimension vectors and the parameter space associated to the cyclic quiver with $l$ vertices (see $4.8, \$ 4.9$. We apply this action to the dimension vector $n \delta$ and the parameter $-\frac{\mathbf{1}}{\mathbf{2}}:=-\frac{1}{2 l}(1, \ldots, 1)$. Fix $w \in \tilde{S}_{l}$ and set $\theta:=w^{-1} \cdot\left(-\frac{\mathbf{1}}{\mathbf{2}}\right)$ and $\gamma:=w * n \delta$. Then $\gamma=n \delta+\gamma_{0}$, where $\gamma_{0}$ is the $l$-residue of a uniquely determined $l$-core partition $\nu$. Set $K:=n l+|\nu|$. The relation between the parameters $\mathbf{h}$ and $\theta$ is explained in $\$ 3.3$.

Both $\mathcal{Y}_{\mathbf{h}}$ and $\mathrm{Hil}_{K}$ carry natural $\mathbb{C}^{*}$-actions with respect to which (1) is equivariant. It follows from 15 that the closed $\mathbb{C}^{*}$-fixed points in $\mathcal{Y}_{\mathbf{h}}$ are labelled by $l$-multipartitions of $n$. On the other hand, the $\mathbb{C}^{*}$-fixed points in $\mathrm{Hil}_{K}$ correspond to monomial ideals in $\mathbb{C}[x, y]$ of colength $K$ and are therefore labelled by partitions of $K$. In particular, the $\mathbb{C}^{*}$ fixed points in $\mathrm{Hil}_{K}^{\nu}$ are labelled by partitions of $K$ with $l$-core $\nu$. Since (1) is equivariant, it induces a bijection

$$
\mathcal{P}(l, n) \longleftrightarrow \mathcal{P}_{\nu}(K)
$$

where $\mathcal{P}_{\nu}(K)$ denotes the set of partitions of $K$ with $l$-core $\nu$ and $\mathcal{P}(l, n)$ the set of $l$ multipartitions of $n$.

Our main result is an explicit combinatorial description of (2). We note that such a description already appeared in [16, but its proof in loc. cit. is incorrect (see Remark 10.5). Our result has a number of interesting applications. We use it to establish a higherlevel version of the combinatorial identity called the $q$-hook formula - a $q$-analogue of the well-known relationship between the dimension of a Specht module and hook lengths in the corresponding Young diagram. Our main result has also recently been used by Bonnafé and Maksimau 3 in their study of fixed-point subvarieties in Calogero-Moser spaces. Moreover, the combinatorial description of (2) is a key ingredient in several older results, such as Bezrukavnikov and Finkelberg's 4], as well as Losev's [26, proofs of Haiman's wreath Macdonald positivity conjecture.

1.1. Main results. Our first result gives a classification as well as an explicit description of $\mathbb{C}^{*}$-fixed points in quiver varieties associated to the cyclic quiver. We also consider tautological bundles on these varieties and calculate the characters of their fibres at the $\mathbb{C}^{*}$-fixed points.

Theorem 1.1. Let $u \in \tilde{S}_{l}, \xi:=u * n \delta=n \delta+\xi_{0}$ and let $\omega$ be the transpose of the l-core corresponding to $\xi_{0}$. Set $L:=n l+|\omega|$. Let $\alpha \in \mathbb{Q}^{l}$ be any parameter such that $\mathcal{X}_{\alpha}(\xi)$ is smooth. Let $\mathcal{V}_{\alpha}(\xi)$ denote the tautological bundle on $\mathcal{X}_{\alpha}(\xi)$ (see 3.2). Then:

a) The $\mathbb{C}^{*}$-fixed points in $\mathcal{X}_{\alpha}(\xi)$ are naturally labelled by $\mathcal{P}_{\omega}(L)$. We construct them explicitly as equivalence classes of quiver representations.

b) Let $\mu \in \mathcal{P}_{\omega}(L)$. Then the $\mathbb{C}^{*}$-character of the fibre of $\mathcal{V}_{\alpha}(\xi)$ at $\mu$ is given by

$$
\operatorname{ch}_{t} \mathcal{V}_{\alpha}(\xi)_{\mu}=\operatorname{Res}_{\mu}(t):=\sum_{\square \in \mu} t^{c(\square)}
$$

This theorem combines the results of Theorem 5.14, Proposition 5.15 and Corollary 5.16 below. Let us briefly explain our description of the $\mathbb{C}^{*}$-fixed points. To each partition $\mu \in \mathcal{P}_{\omega}(L)$ we associate a quadruple of matrices depending on $\alpha, \xi$ and the Frobenius form of $\mu$. Our construction can be regarded as a generalization of Wilson's description of the $\mathbb{C}^{*}$-fixed points in the (classical) Calogero-Moser space [36, Proposition 6.11]. One can also interpret our construction in terms of unravelling the cyclic quiver into the infinite linear quiver $A_{\infty}$. This connects our results with earlier work on quiver varieties of type $A_{\infty}[13,34$.

Our second result describes the bijection between the $\mathbb{C}^{*}$-fixed points induced by the Etingof-Ginzburg isomorphism.

Theorem 1.2 (Theorem 7.18). The map $\mathcal{Y}_{\mathbf{h}} \stackrel{\mathrm{EG}}{\longrightarrow} \mathcal{X}_{\theta}(n \delta)$ induces a bijection

$$
\mathcal{P}(l, n) \rightarrow \mathcal{P}_{\varnothing}(n l), \quad \underline{\mathrm{Quot}}(\mu)^{b} \mapsto \mu,
$$


where Quot $(\mu)^{b}$ denotes the reverse of the l-quotient of $\mu$ (see \$2.1. \$4.5) and $\varnothing$ is the empty partition.

The proof of Theorem 1.2 occupies sections 6 and 7 . We use the Dunkl-Opdam subalgebra of $\mathbb{H}_{\mathbf{h}}$ to construct a commutative diagram

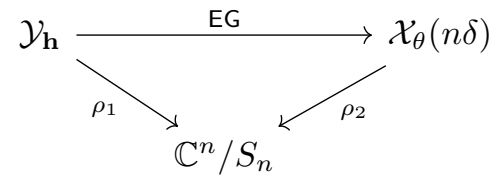

where $\rho_{1}$ sends a fixed point labelled by $\underline{\lambda}$ to its residue and $\rho_{2}$ sends a quiver representation to a certain subset of its eigenvalues. Given a partition $\mu$, we use our description of the corresponding fixed point from Theorem 1.1 to obtain an explicit formula for $\rho_{2}(\mu)$ in terms of the Frobenius form of $\mu$. We then use a combinatorial argument to show that this formula describes the residue of Quot $(\mu)^{b}$.

We next consider reflection functors, which were introduced by Nakajima 30, and also studied by Maffei [27] and Crawley-Boevey and Holland [7, 8]. Assuming smoothness, reflection functors are $U(1)$-equivariant hyper-Kähler isometries between quiver varieties associated to different parameters. They satisfy Weyl group relations and have been used by Nakajima to define Weyl group representations on homology groups of quiver varieties.

Let us explain the role reflection functors play in our setting. To each simple reflection $\sigma_{i} \in \tilde{S}_{l}$, we associate a reflection functor $\mathfrak{R}_{i}: \mathcal{X}_{\alpha}(\xi) \rightarrow \mathcal{X}_{\sigma_{i} \cdot \alpha}\left(\sigma_{i} * \xi\right)$. One can show that $\sigma_{i} * \xi=n \delta+\sigma_{i} * \xi_{0}$, where $\sigma_{i} * \xi_{0}$ is the $l$-residue of a uniquely determined $l$-core $\omega^{\prime}$. Then the reflection functor $\mathfrak{R}_{i}$ induces a bijection between the labelling sets of $\mathbb{C}^{*}$-fixed points

$$
\mathbf{R}_{i}: \mathcal{P}_{\omega}(L) \rightarrow \mathcal{P}_{\left(\omega^{\prime}\right)^{t}}\left(L^{\prime}\right),
$$

where $L^{\prime}:=n l+\left|\omega^{\prime}\right|$.

Our third result gives a combinatorial description of this bijection. We use the action of $\tilde{S}_{l}$ on the set of all partitions defined by Van Leeuwen in [25]. This action involves combinatorial ideas reminiscent of those describing the $\hat{\mathfrak{s l}}_{l}$-action on the Fock space. More precisely, if $\mu$ is a partition then $\sigma_{i} * \mu$ is the partition obtained by simultaneously removing and adding all the removable (resp. addable) cells of content $i \bmod l$ from (to) the Young diagram of $\mu$. It is noteworthy that this action also plays a role in the combinatorics describing the Schubert calculus of the affine Grassmannian [23, 24].

Theorem 1.3 (Theorem 9.11). Let $\mu \in \mathcal{P}_{\omega}(L)$. Then

$$
\mathbf{R}_{i}(\mu)=\left(\sigma_{i} * \mu^{t}\right)^{t} .
$$

Combining Theorem 1.2 with (iterated applications of) Theorem 1.3 allows us to give an explicit combinatorial description of bijection (2).

Theorem 1.4 (Theorem 10.3). The map (1) induces the following bijections

$$
\begin{aligned}
& \mathcal{Y}_{\mathbf{h}}^{\mathbb{C}^{*}} \longrightarrow \mathcal{X}_{\theta}(n \delta)^{\mathbb{C}^{*}} \longrightarrow \mathcal{X}_{-\frac{1}{2}}(\gamma)^{\mathbb{C}^{*}} \longrightarrow\left(\mathrm{Hil}_{K}^{\nu}\right)^{\mathbb{C}^{*}} \\
& \mathcal{P}(l, n) \longrightarrow \mathcal{P}_{\varnothing}(n l) \longrightarrow \mathcal{P}_{\nu^{t}}(K) \quad \longrightarrow \mathcal{P}_{\nu}(K) \\
& \underline{Q u o t}(\mu)^{b} \longmapsto \quad \mu \quad \longmapsto \quad\left(w * \mu^{t}\right)^{t} \longmapsto w * \mu^{t} .
\end{aligned}
$$

Moreover, $\nu=w * \varnothing$.

Let us rephrase our result slightly. Given $w \in \tilde{S}_{l}$, we define the $w$-twisted l-quotient bijection to be the map

$$
\tau_{w}: \mathcal{P}(l, n) \rightarrow \mathcal{P}_{\nu}(K), \quad \underline{\operatorname{Quot}}(\mu) \mapsto w * \mu .
$$

Corollary 1.5 (Corollary 10.4). Bijection (2) is given by

$$
\underline{\lambda} \mapsto \tau_{w}\left(\underline{\lambda}^{t}\right) .
$$

One of Gordon's motivations in [16] was to give a geometric interpretation of highest weight structures on category $\mathcal{O}_{\mathbf{h}}$ for rational Cherednik algebras $\mathbb{H}_{t=1, \mathbf{h}}\left(\Gamma_{n}\right)$ at $t=1$. Consider the combinatorial ordering $\prec_{\mathbf{h}}^{\text {com }}$ on $\mathcal{P}(l, n)$ defined by

$$
\underline{\mu} \preceq_{\mathbf{h}}^{\mathrm{com}} \underline{\lambda} \Longleftrightarrow \tau_{w}\left(\underline{\lambda}^{t}\right) \unlhd \tau_{w}\left(\underline{\mu}^{t}\right),
$$


where $\unlhd$ denotes the dominance ordering on partitions. It was shown by Dunkl and Griffeth [10, Theorem 1.2] that $\mathcal{O}_{\mathbf{h}}$ is a highest weight category with respect to this ordering. There is also a geometric ordering $\prec_{\mathbf{h}}^{\text {geo }}$ on $\mathcal{P}(l, n)$, defined by the closure relations between the attracting sets of $\mathbb{C}^{*}$-fixed points in $\mathcal{M}_{2 \theta}(n \delta)$. Using Corollary 1.5 and the results of Nakajima from [29] we deduce the following partial geometric interpretation of the combinatorial ordering.

Corollary 1.6 (Corollary 10.6). Let $\underline{\mu}, \underline{\lambda} \in \mathcal{P}(l, n)$. Then $\underline{\mu} \preceq_{\mathbf{h}}^{\text {geo }} \underline{\lambda} \Rightarrow \underline{\mu} \preceq_{\mathbf{h}}^{\text {com }} \underline{\lambda}$.

We remark that the statements of Corollaries 1.5 and 1.6 first appeared in [16] (see Proposition 7.10 and its proof). However, the proof of Proposition 7.10 in [16] is incorrect - see Remark 10.5 for an explanation.

1.2. The higher-level $q$-hook formula. Our results have several interesting applications. One of them is a new proof as well as a generalization of the $q$-hook formula:

$$
\sum_{\square \in \mu} t^{c(\square)}=[n]_{t} \sum_{\lambda \uparrow \mu} \frac{f_{\lambda}(t)}{f_{\mu}(t)},
$$

where $\mu$ is a partition of $n, c(\square)$ is the content of $\square$ and $f_{\mu}(t)$ is the fake degree polynomial associated to $\mu$. The sum on the RHS ranges over subpartitions of $\mu$ obtained by deleting precisely one cell in the Young diagram of $\mu$. Note that the RHS of (4) can also be reformulated in terms of Schur functions and hook length polynomials. The $q$-hook formula has been proven by Kerov [22, Garsia and Haiman [14] and Chen and Stanley [6] using probabilistic, combinatorial and algebraic methods. We prove the following generalization.

Theorem 1.7 (Theorem 8.1). Let $\mu \in \mathcal{P}_{\varnothing}(n l)$. Then:

$$
\sum_{\square \in \mu} t^{c(\square)}=[n l]_{t} \sum_{\underline{\lambda \uparrow} \underline{\operatorname{Quot}(\mu)^{b}}} \frac{f_{\underline{\lambda}}(t)}{f_{\text {Quot }}(\mu)^{b}(t)} .
$$

We call (5) the higher-level $q$-hook formula. Setting $l=1$ we recover the classical $q$-hook formula. Our proof of Theorem 1.7 is geometric in nature. Let us briefly explain the main idea behind it. Let $e_{n}$ denote the symmetrizing idempotent in $\Gamma_{n}$. The right $e_{n} \mathbb{H}_{\mathbf{h}} e_{n^{-}}$ module $\mathbb{H}_{\mathbf{h}} e_{n}$ defines a coherent sheaf on $\mathcal{Y}_{\mathbf{h}}$. Since we are assuming that the variety $\mathcal{Y}_{\mathbf{h}}$ is smooth, this sheaf is also locally free. Let $\mathcal{R}_{\mathbf{h}}$ denote the corresponding vector bundle. It was shown in [12] that there exists an isomorphism of vector bundles $\mathcal{R}_{\mathbf{h}}^{\Gamma_{n-1}} \stackrel{\sim}{\rightarrow} \mathcal{V}_{\theta}(n \delta)$ lifting the Etingof-Ginzburg isomorphism $\mathcal{Y}_{\mathbf{h}} \stackrel{\sim}{\longrightarrow} \mathcal{X}_{\theta}(n \delta)$. Let $\mu \in \mathcal{P}_{\varnothing}(n l)$. By Theorem 1.2 the Etingof-Ginzburg map sends the fixed point labelled by Quot $(\mu)^{b}$ to the fixed point labelled by $\mu$. We obtain the higher level $q$-hook formula $\sqrt{5}$ by comparing the $\mathbb{C}^{*}$-characters of the corresponding fibres $\left(\mathcal{R}_{\mathbf{h}}^{\Gamma_{n-1}}\right)_{\underline{\text { Quot }}(\mu)^{b}}$ and $\mathcal{V}_{\theta}(n \delta)_{\mu}$.

1.3. Other applications. Let us mention a few other applications of our results. The first is related to Finkelberg and Bezrukavnikov's [4] as well as Losev's [26] proofs of Haiman's wreath Macdonald positivity conjecture. The original positivity conjecture, proven by Haiman in [19], asserts that Kostka-Macdonald polynomials, which express the change of basis between transformed Macdonald functions and Schur functions, have non-negative coefficients. Haiman [20] later proposed a generalized conjecture, known as the wreath Macdonald positivity conjecture, in which the ring of symmetric functions is replaced by the space of virtual characters of the group $\Gamma_{n}$.

We will now explain the role the description of the bijection (2) from Corollary 1.5 plays in the above-mentioned proofs of the wreath Macdonald positivity conjecture. The key step in Bezrukavnikov and Finkelberg's proof is a characterization of the support of certain Verma modules in positive characteristic [4, Proposition 2.6]. Losev's proof also relies on a calculation of the supports of certain quotients of Procesi bundles [26, Proposition 5.3]. The proofs of these two statements invoke [4, Lemma 3.8]. But the latter implicitly uses Corollary 1.5 (see also [4, §2.3]).

We mention two other applications of ours results. Gordon and Martino [17] gave a combinatorial description of the blocks of the restricted rational Cherednik algebra of type $G(l, 1, n)$ (also for parameters $\mathbf{h}$ for which the corresponding Calogero-Moser space $\mathcal{Y}_{\mathbf{h}}$ is singular) in terms of $J$-classes of partitions. Corollary 1.5 is an important ingredient in their proof. 
More recently, Bonnafé and Maksimau [3] studied the irreducible components of the fixed point subvariety under the action of a finite cyclic group on a smooth CalogeroMoser space. They use Theorem 1.2 to give an explicit description of these components for Calogero-Moser spaces of type $G(l, 1, n)$.

Acknowledgements. The research for this paper was carried out with the financial support of the College of Science \& Engineering at the University of Glasgow. The material will form part of my PhD thesis. I would like to thank G. Bellamy for suggesting the problem to me as well as his support and encouragement. I am also grateful to C. Stroppel for comments on a draft version of this paper and to I. Gordon for discussing [16] with me.

\section{Rational Cherednik algebras}

We begin by recalling the definition and basic properties of cyclotomic rational Cherednik algebras at $t=0$. We work over the field of complex numbers throughout the paper.

2.1. Partitions and multipartitions. Let $k$ be a non-negative integer. A partition $\lambda$ of $k$ is an infinite non-increasing sequence $\left(\lambda_{1}, \lambda_{2}, \lambda_{3}, \ldots\right)$ of non-negative integers such that $\sum_{i=1}^{\infty} \lambda_{i}=k$. We write $|\lambda|=k$ and denote the set of all partitions of $k$ by $\mathcal{P}(k)$. Let $\ell(\lambda)$ be the positive integer $i$ such that $\lambda_{i} \neq 0$ but $\lambda_{i+1}=0$. We say that $\mu=\left(\mu_{1}, \mu_{2}, \mu_{3}, \ldots\right)$ is a subpartition of $\lambda$ if $\mu$ is a partition of some positive integer $m \leq k$ and $\mu_{i} \leq \lambda_{i}$ for all $i=1,2, \ldots$. A subpartition $\mu$ of $\lambda$ is called a restriction of $\lambda$, denoted $\mu \uparrow \lambda$, if $|\mu|=k-1$. Let $\varnothing=(0,0, \ldots)$ denote the empty partition.

An $l$-composition $\alpha$ of $k$ is an $l$-tuple $\left(\alpha_{0}, \ldots, \alpha_{l-1}\right)$ of non-negative integers such that $\sum_{i=0}^{l-1} \alpha_{i}=k$. An l-multipartition $\underline{\lambda}$ of $k$ is an $l$-tuple $\left(\lambda^{0}, \ldots, \lambda^{l-1}\right)$ such that each $\lambda^{i}$ is a partition and $\sum_{i=0}^{l-1}\left|\lambda^{i}\right|=k$. We consider the upper indices modulo $l$. Let $\mathcal{P}(l, k)$ denote the set of $l$-multipartitions of $k$. We say that $\underline{\mu}=\left(\mu^{0}, \ldots, \mu^{l-1}\right)$ is a submultipartition of $\underline{\lambda}$ if each $\mu^{i}$ is a subpartition of $\lambda^{i}$. We call a submultipartition $\underline{\mu}$ of $\underline{\lambda}$ a restriction of $\underline{\lambda}$, denoted $\underline{\mu} \uparrow \underline{\lambda}$, if $\sum_{i=0}^{l-1}\left|\mu^{i}\right|=k-1$.

If $\lambda$ is a partition we denote its transpose by $\lambda^{t}$. If $\underline{\lambda}=\left(\lambda^{0}, \ldots, \lambda^{l-1}\right) \in \mathcal{P}(l, k)$, we call $\underline{\lambda}^{t}=\left(\left(\lambda^{0}\right)^{t}, \ldots,\left(\lambda^{l-1}\right)^{t}\right)$ the transpose multipartition and $\underline{\lambda}^{b}:=\left(\lambda^{l-1}, \lambda^{l-2}, \ldots, \lambda^{0}\right)$ the reverse multipartition. Finally, we set $\mathcal{P}:=\bigsqcup_{k \in \mathbb{Z}_{\geq 0}} \mathcal{P}(k)$ and $\mathcal{P}:=\bigsqcup_{k \in \mathbb{Z}_{\geq 0}} \mathcal{P}(l, k)$.

2.2. Wreath products. Let us fix once and for all two positive integers $n, l$. We regard the symmetric group $S_{n}$ as the group of permutations of the set $\{1, \ldots, n\}$. For $1 \leq$ $i<j \leq n$ let $s_{i, j}$ denote the transposition swapping numbers $i$ and $j$. We abbreviate $s_{i}=s_{i, i+1}$ for $i=1, \ldots, n-1$. Let $C_{l}:=\mathbb{Z} / l \mathbb{Z}=\langle\epsilon\rangle$ and set $\Gamma_{n}:=C_{l}\left\langle S_{n}=\left(C_{l}\right)^{n} \rtimes S_{n}\right.$, the wreath product of $C_{l}$ and $S_{n}$. It is a complex reflection group of type $G(l, 1, n)$. For $1 \leq i \leq n$ and $1 \leq j \leq l-1$ let $\epsilon_{i}^{j}$ denote the element $\left(1, \ldots, 1, \epsilon^{j}, 1, \ldots, 1\right) \in\left(C_{l}\right)^{n}$ which is non-trivial only in the $i$-th coordinate. Let $e_{n}:=\left(l^{n} n !\right)^{-1} \sum_{g \in \Gamma_{n}} g$ be the symmetrizing idempotent and let triv denote the trivial $\Gamma_{n}$-module.

We regard $S_{n-1}$ as the subgroup of $S_{n}$ generated by the transpositions $s_{2}, \ldots, s_{n-1}$. We also regard $\left(C_{l}\right)^{n-1}$ as a subgroup of $\left(C_{l}\right)^{n}$ consisting of elements whose first coordinate is equal to one. This determines an embedding $\Gamma_{n-1} \hookrightarrow \Gamma_{n}$. Note that $\left(\mathbb{C} \Gamma_{n}\right)^{\Gamma_{n-1}}=$ $e_{n-1} \mathbb{C} \Gamma_{n}$ and $\left|\left(\mathbb{C} \Gamma_{n}\right)^{\Gamma_{n-1}}\right|=n l$.

Isomorphism classes of irreducible $\Gamma_{n}$-modules are naturally parametrized by $\mathcal{P}(l, n)$. We use the parametrization given in $33, \S 6.1 .1]$. Let $S(\underline{\lambda})$ denote the irreducible $\Gamma_{n^{-}}$ module corresponding to the $l$-multipartition $\underline{\lambda}$. We will later need the following branching rule [31, Theorem 10].

Proposition 2.1. Let $\underline{\lambda} \in \mathcal{P}(l, n)$. Then $\left.S(\underline{\lambda})\right|_{\Gamma_{n-1}}:=\operatorname{Res}_{\Gamma_{n-1}}^{\Gamma_{n}} S(\underline{\lambda})=\bigoplus_{\underline{\mu} \uparrow \underline{\underline{\lambda}}} S(\underline{\mu})$.

2.3. Rational Cherednik algebras. Let us recall the definition of the rational Cherednik algebra of type $G(l, 1, n)$ at $t=0$. Set $\eta:=e^{2 \pi i / l}$. Let $\mathfrak{h}$ be the $n$-dimensional representation of $\Gamma_{n}$ with basis $y_{1}, \ldots, y_{n}$ such that $\epsilon_{i} \sigma \cdot y_{j}=\eta^{-\delta_{i, \sigma(j)}} y_{\sigma(j)}$ for any $\sigma \in S_{n}$. Let $x_{1}, \ldots, x_{n}$ be the dual basis of $\mathfrak{h}^{*}$. 
Definition 2.2. Let us choose a parameter $\mathbf{h}=\left(h, H_{1}, \ldots, H_{l-1}\right) \in \mathbb{Q}^{l}$ and set $H_{0}=$ $-\left(H_{1}+\ldots+H_{l-1}\right)$. The rational Cherednik algebra $\mathbb{H}_{\mathbf{h}}$ (at $\left.t=0\right)$ associated to $\Gamma_{n}$ is the quotient of the cross-product $T\left(\mathfrak{h} \oplus \mathfrak{h}^{*}\right) \rtimes \mathbb{C} \Gamma_{n}$ by the relations

- $\left[x_{i}, x_{j}\right]=\left[y_{i}, y_{j}\right]=0$ for all $1 \leq i, j \leq n$,

- $\left[x_{i}, y_{j}\right]=-h \sum_{k=0}^{l-1} \eta^{k} s_{i, j} \epsilon_{i}^{k} \epsilon_{j}^{-k}$ for all $1 \leq i \neq j \leq n$,

- $\left[x_{i}, y_{i}\right]=h \sum_{j \neq i} \sum_{k=0}^{l-1} s_{i, j} \epsilon_{i}^{k} \epsilon_{j}^{-k}+\sum_{k=0}^{l-1}\left(\sum_{m=0}^{l-1} \eta^{-m k} H_{m}\right) \epsilon_{i}^{k}$ for all $1 \leq i \leq n$.

Let $Z_{\mathbf{h}}$ denote the centre of $\mathbb{H}_{\mathbf{h}}$. By [12, Theorem 3.1], the map

$$
Z_{\mathbf{h}} \rightarrow e_{n} \mathbb{H}_{\mathbf{h}} e_{n}, z \mapsto z \cdot e_{n}
$$

is an algebra isomorphism. It is called the Satake isomorphism. Consider the $\mathbb{C}^{*}$-action on $\mathbb{H}_{\mathbf{h}}$ defined by the rule $t . x_{i}=t x_{i}, t . y_{i}=t^{-1} y_{i}$ and $t . g=g$, where $1 \leq i \leq n, g \in \Gamma_{n}$ and $t \in \mathbb{C}^{*}$. This action restricts to actions on $e_{n} \mathbb{H}_{\mathbf{h}} e_{n}$ and $Z_{\mathbf{h}}$, with respect to which the Satake isomorphism is equivariant. Note that the $\mathbb{C}^{*}$-action on $\mathbb{H}_{\mathbf{h}}$ can also be interpreted as a $\mathbb{Z}$-grading such that $\operatorname{deg} x_{i}=1, \operatorname{deg} y_{i}=-1$ and $\operatorname{deg} g=0$.

Notation. Given a $\mathbb{Z}$-graded vector space $V$ with finite-dimensional homogeneous components, let $\operatorname{ch}_{t} V \in \mathbb{Z}\left[\left[t, t^{-1}\right]\right]$ denote its Poincaré series. Since a $\mathbb{Z}$-grading is equivalent to a $\mathbb{C}^{*}$-module structure, we can regard $\operatorname{ch}_{t} V$ as the $\mathbb{C}^{*}$-character of $V$.

Definition 2.3. Let $\mathbb{C}[\mathfrak{h}]_{+}^{\Gamma_{n}}$ (resp. $\mathbb{C}\left[\mathfrak{h}^{*}\right]_{-}^{\Gamma_{n}}$ ) denote the ideal of $\mathbb{C}[\mathfrak{h}]^{\Gamma_{n}}$ (resp. $\mathbb{C}\left[\mathfrak{h}^{*}\right]^{\Gamma_{n}}$ ) generated by homogeneous elements of positive (resp. negative) degree, in the grading defined by the $\mathbb{C}^{*}$-action on $\mathbb{H}_{\mathbf{h}}$. The quotient

$$
\overline{\mathbb{H}}_{\mathbf{h}}:=\mathbb{H}_{\mathbf{h}} / \mathbb{H}_{\mathbf{h}} \cdot\left(\mathbb{C}[\mathfrak{h}]_{+}^{\Gamma_{n}}+\mathbb{C}\left[\mathfrak{h}^{*}\right]_{-}^{\Gamma_{n}}\right)
$$

is called the restricted rational Cherednik algebra. It is a finite-dimensional algebra.

Let $\mathbb{C}[\mathfrak{h}]^{c o \Gamma_{n}}:=\mathbb{C}[\mathfrak{h}] / \mathbb{C}[\mathfrak{h}] . \mathbb{C}[\mathfrak{h}]_{+}^{\Gamma_{n}}$ be the algebra of coinvariants with respect to the $\Gamma_{n^{-}}$ action. It follows from the PBW theorem for rational Cherednik algebras [12, Theorem 1.3] that there is an isomorphism of graded vector spaces $\overline{\mathbb{H}}_{\mathbf{h}} \cong \mathbb{C}[\mathfrak{h}]^{c o \Gamma_{n}} \otimes \mathbb{C}\left[\mathfrak{h}^{*}\right]^{c o \Gamma_{n}} \otimes \mathbb{C} \Gamma_{n}$. Moreover, $\mathbb{C}\left[\mathfrak{h}^{*}\right]^{c o \Gamma_{n}} \rtimes \mathbb{C} \Gamma_{n}$ is a subalgebra of $\overline{\mathbb{H}}_{\mathbf{h}}$.

Definition 2.4. Let $\underline{\lambda} \in \mathcal{P}(l, n)$. The irreducible $\mathbb{C} \Gamma_{n}$-module $S(\underline{\lambda})$ becomes a module over $\mathbb{C}\left[\mathfrak{h}^{*}\right]^{c o \Gamma_{n}} \rtimes \mathbb{C} \Gamma_{n}$ by means of the projection $\mathbb{C}\left[\mathfrak{h}^{*}\right]^{c o \Gamma_{n}} \rtimes \mathbb{C} \Gamma_{n} \rightarrow \mathbb{C} \Gamma_{n}$. The baby Verma module associated to $\lambda$ is the induced module

$$
\Delta(\underline{\lambda}):=\overline{\mathbb{H}}_{\mathbf{h}} \otimes_{\left.\mathbb{C}_{\left[\mathfrak{h}^{*}\right.}\right]^{c o \Gamma_{n}} \rtimes \mathbb{C} \Gamma_{n}} S(\underline{\lambda}) .
$$

We consider $\Delta(\underline{\lambda})$ as a graded $\overline{\mathbb{H}}_{\mathbf{h}}$-module with $1 \otimes S(\underline{\lambda})$ in degree 0 .

Proposition 2.5 ([15, Proposition 4.3]). Let $\underline{\lambda} \in \mathcal{P}(l, n)$. The baby Verma module $\Delta(\underline{\lambda})$ is indecomposable with simple head $L(\underline{\lambda})$. Moreover, $\{L(\underline{\lambda}) \mid \underline{\lambda} \in \mathcal{P}(l, n)\}$ form a complete and irredundant set of representatives of isomorphism classes of graded simple $\overline{\mathbb{H}}_{\mathbf{h}}$ modules, up to a grading shift.

2.4. The variety $\mathcal{Y}_{\mathbf{h}}$. Let $\mathcal{Y}_{\mathbf{h}}:=\operatorname{Spec} Z_{\mathbf{h}}$. We will always assume that the parameter $\mathbf{h}$ is chosen so that the variety $\mathcal{Y}_{\mathbf{h}}$ is smooth. A criterion for smoothness can be found in e.g. [16. Lemma 4.3].

Let $\operatorname{Irrep}\left(\mathbb{H}_{\mathbf{h}}\right)$ denote the set of isomorphism classes of irreducible representations of $\mathbb{H}_{\mathbf{h}}$. If $[M] \in \operatorname{Irrep}\left(\mathbb{H}_{\mathbf{h}}\right)$, let $\chi_{M}: Z_{\mathbf{h}} \rightarrow \mathbb{C}$ denote the character by which $Z_{\mathbf{h}}$ acts on $M$. By [12, Theorem 1.7], there is a bijection

$$
\operatorname{Irrep}\left(\mathbb{H}_{\mathbf{h}}\right) \longleftrightarrow \operatorname{MaxSpec} Z_{\mathbf{h}}, \quad[M] \mapsto \operatorname{ker} \chi_{M}
$$

We are now going to recall another description of $\mathcal{Y}_{\mathbf{h}}$.

Definition 2.6. Let $\operatorname{Rep}_{\mathbb{C}_{n}}\left(\mathbb{H}_{\mathbf{h}}\right)$ be the variety of all algebra homomorphisms $\mathbb{H}_{\mathbf{h}} \rightarrow$ $\operatorname{End}_{\mathbb{C}}\left(\mathbb{C} \Gamma_{n}\right)$ whose restriction to $\mathbb{C} \Gamma_{n} \subset \mathbb{H}_{\mathbf{h}}$ is the $\mathbb{C} \Gamma_{n}$-action by left multiplication, i.e., the regular representation. This is an affine algebraic variety.

Let $\phi \in \operatorname{Rep}_{\mathbb{C} \Gamma_{n}}\left(\mathbb{H}_{\mathbf{h}}\right)$. The one-dimensional vector space $e_{n} \mathbb{C} \Gamma_{n}$ is stable under all the endomorphisms in $\phi\left(e_{n} \mathbb{H}_{\mathbf{h}} e_{n}\right)$. Therefore, $\left.\phi\right|_{e_{n} \mathbb{H}_{\mathbf{h}} e_{n}}$ composed with the Satake isomorphism yields an algebra homomorphism $\chi_{\phi}: Z_{\mathbf{h}} \cong e_{n} \mathbb{H}_{\mathbf{h}} e_{n} \rightarrow \operatorname{End}_{\mathbb{C}}\left(e_{n} \mathbb{C} \Gamma_{n}\right) \cong \mathbb{C}$. We obtain a morphism of algebraic varieties

$$
\pi: \operatorname{Rep}_{\mathbb{C} \Gamma_{n}}\left(\mathbb{H}_{\mathbf{h}}\right) \rightarrow \mathcal{Y}_{\mathbf{h}}, \quad \phi \mapsto \operatorname{ker} \chi_{\phi}
$$


The $\mathbb{C}^{*}$-action on $\mathbb{H}_{\mathbf{h}}$ induces $\mathbb{C}^{*}$-actions on the varieties $\operatorname{Rep}_{\mathbb{C}_{n}}\left(\mathbb{H}_{\mathbf{h}}\right)$ and $\mathcal{Y}_{\mathbf{h}}$, with respect to which $\pi$ is equivariant.

Let $\operatorname{Aut}_{\Gamma_{n}}\left(\mathbb{C} \Gamma_{n}\right)$ be the group of $\mathbb{C}$-linear $\Gamma_{n}$-equivariant automorphisms of $\mathbb{C} \Gamma_{n}$. The group $\operatorname{Aut}_{\Gamma_{n}}\left(\mathbb{C} \Gamma_{n}\right)$ acts naturally on $\operatorname{Rep}_{\mathbb{C}_{n}}\left(\mathbb{H}_{\mathbf{h}}\right)$ : if $g \in \operatorname{Aut}_{\Gamma_{n}}\left(\mathbb{C} \Gamma_{n}\right)$ and $\phi \in \operatorname{Rep}_{\mathbb{C}_{n}}\left(\mathbb{H}_{\mathbf{h}}\right)$ then $(g . \phi)(z)=g \phi(z) g^{-1}$, for all $z \in \mathbb{H}_{\mathbf{h}}$. By [12, Theorem 3.7], there exists an irreducible component $\operatorname{Rep}_{\mathbb{C} \Gamma_{n}}^{o}\left(\mathbb{H}_{\mathbf{h}}\right)$ of $\operatorname{Rep}_{\mathbb{C} \Gamma_{n}}\left(\mathbb{H}_{\mathbf{h}}\right)$ such that 7 induces a $\mathbb{C}^{*}$-equivariant isomorphism of algebraic varieties

$$
\operatorname{Rep}_{\mathbb{C} \Gamma_{n}}^{o}\left(\mathbb{H}_{\mathbf{h}}\right) / / \operatorname{Aut}_{\Gamma_{n}}\left(\mathbb{C} \Gamma_{n}\right) \stackrel{\sim}{\rightarrow} \mathcal{Y}_{\mathbf{h}}
$$

Next, consider the $\left(\mathbb{H}_{\mathbf{h}}, e_{n} \mathbb{H}_{\mathbf{h}} e_{n}\right)$-bimodule $\mathbb{H}_{\mathbf{h}} e_{n}$ together with the $\mathbb{C}^{*}$-action inherited from $\mathbb{H}_{\mathbf{h}}$. The bimodule $\mathbb{H}_{\mathbf{h}} e_{n}$ defines a $\mathbb{C}^{*}$-equivariant coherent sheaf $\widetilde{\mathbb{H}_{\mathbf{h}} e_{n}}$ on Spec $e_{n} \mathbb{H}_{\mathbf{h}} e_{n} \cong \mathcal{Y}_{\mathbf{h}}$. Since we are assuming that $\mathcal{Y}_{\mathbf{h}}$ is smooth, [12, Theorem 1.7] implies that this sheaf is locally free.

Definition 2.7. Let $\mathcal{R}_{\mathbf{h}}$ denote the $\mathbb{C}^{*}$-equivariant vector bundle whose sheaf of sections is $\widetilde{\mathbb{H}_{\mathbf{h}} e_{n}}$.

The group $\Gamma_{n}$ acts naturally on every fibre of $\mathcal{R}_{\mathbf{h}}$ from the left. Let $\mathcal{R}_{\mathbf{h}}^{\Gamma_{n-1}}=e_{n-1} \mathcal{R}_{\mathbf{h}}$ be the subbundle of $\mathcal{R}_{\mathbf{h}}$ consisting of $\Gamma_{n-1}$-invariants and let $\left(\mathcal{R}_{\mathbf{h}}^{\Gamma_{n-1}}\right)_{\underline{\lambda}}$ denote its fibre at $\chi_{\underline{\lambda}}$.

2.5. $\mathbb{C}^{*}$-fixed points. Let us recall the classification of $\mathbb{C}^{*}$-fixed points in $\mathcal{Y}_{\mathbf{h}}$ in terms of $l$ multipartitions of $n$ from [15]. By [12, Proposition 4.15], the subalgebra $\mathbb{C}[\mathfrak{h}]^{\Gamma_{n}} \otimes \mathbb{C}\left[\mathfrak{h}^{*}\right]^{\Gamma_{n}}$ of $\mathbb{H}_{\mathbf{h}}$ is contained in $Z_{\mathbf{h}}$ and $Z_{\mathbf{h}}$ is a free $\mathbb{C}[\mathfrak{h}]^{\Gamma_{n}} \otimes \mathbb{C}\left[\mathfrak{h}^{*}\right]^{\Gamma_{n}}$-module of rank $\left|\Gamma_{n}\right|$. The inclusion $\mathbb{C}[\mathfrak{h}]^{\Gamma_{n}} \otimes \mathbb{C}\left[\mathfrak{h}^{*}\right]^{\Gamma_{n}} \hookrightarrow Z_{\mathbf{h}}$ induces a $\mathbb{C}^{*}$-equivariant morphism of algebraic varieties

$$
\Upsilon: \mathcal{Y}_{\mathbf{h}} \rightarrow \mathfrak{h} / \Gamma_{n} \times \mathfrak{h}^{*} / \Gamma_{n}
$$

The only $\mathbb{C}^{*}$-fixed point in $\mathfrak{h} / \Gamma_{n} \times \mathfrak{h}^{*} / \Gamma_{n}$ is 0 . Since the group $\mathbb{C}^{*}$ is connected and the fibre $\Upsilon^{-1}(0)$ is finite, $\mathcal{Y}_{\mathbf{h}}^{\mathbb{C}^{*}}=\Upsilon^{-1}(0)$. By Theorem 5.6 in [15], there is a bijection between the closed points of $\Upsilon^{-1}(0)$ and isomorphism classes of simple modules over the restricted rational Cherednik algebra $\overline{\mathbb{H}}_{\mathbf{h}}$. Hence there is a bijection

$$
\mathcal{P}(l, n) \longleftrightarrow\left(\operatorname{MaxSpec} Z_{\mathbf{h}}\right)^{\mathbb{C}^{*}}, \quad \underline{\lambda} \mapsto \operatorname{ker} \chi_{L(\underline{\lambda})}
$$

We will also write $\chi_{\underline{\lambda}}$ for $\chi_{L(\underline{\lambda})}$.

\section{Quiver VARIETIES}

In this section we recall the connection between rational Cherednik algebras and cyclic quiver varieties via the Etingof-Ginzburg isomorphism.

3.1. The cyclic quiver. Let $\mathbf{Q}$ be the cyclic quiver with $l$ vertices and a cyclic orientation. We label the vertices as $0,1, \ldots, l-1$ (considered as elements of $\mathbb{Z} / l \mathbb{Z}$ ) in such a way that there is a (unique) arrow $i \rightarrow j$ if and only if $j=i+1$. Let $\overline{\mathbf{Q}}$ be the double of $\mathbf{Q}$, i.e., the quiver obtained from $\mathbf{Q}$ by adding, for each arrow $a$ in $\mathbf{Q}$, an arrow $a^{*}$ going in the opposite direction. Moreover, let $\mathbf{Q}_{\infty}$ be the quiver obtained from $\mathbf{Q}$ by adding an extra vertex, denoted $\infty$, and an extra arrow $a_{\infty}: \infty \rightarrow 0$. We write $\overline{\mathbf{Q}}_{\infty}$ for the double of $\mathbf{Q}_{\infty}$.

Let $\mathbf{d}=\left(d_{0}, \ldots, d_{l-1}\right) \in\left(\mathbb{Z}_{\geq 0}\right)^{l}$. We interpret $\mathbf{d}$ as the dimension vector for $\overline{\mathbf{Q}}$ so that the dimension associated to the vertex $i$ is $d_{i}$. For each $i=0, \ldots, l-1$ let $\mathbf{V}_{i}$ be a complex vector space of dimension $d_{i}$. Set $\widehat{\mathbf{V}}:=\bigoplus_{i=0}^{l-1} \mathbf{V}_{i}$. Moreover, let $\mathbf{V}_{\infty}$ be a one-dimensional complex vector space and set $\mathbf{V}:=\mathbf{V}_{\infty} \oplus \widehat{\mathbf{V}}$. Define

$\operatorname{Rep}\left(\overline{\mathbf{Q}}_{\infty}, \mathbf{d}\right):=\bigoplus_{i=0}^{l-1} \operatorname{Hom}\left(\mathbf{V}_{i}, \mathbf{V}_{i+1}\right) \oplus \bigoplus_{i=0}^{l-1} \operatorname{Hom}\left(\mathbf{V}_{i}, \mathbf{V}_{i-1}\right) \oplus \operatorname{Hom}\left(\mathbf{V}_{0}, \mathbf{V}_{\infty}\right) \oplus \operatorname{Hom}\left(\mathbf{V}_{\infty}, \mathbf{V}_{0}\right)$

We denote an element of $\operatorname{Rep}\left(\overline{\mathbf{Q}}_{\infty}, \mathbf{d}\right)$ as $(\mathbf{X}, \mathbf{Y}, I, J)=\left(X_{0}, \ldots, X_{l-1}, Y_{0}, \ldots, Y_{l-1}, I, J\right)$ accordingly. There is a natural isomorphism of varieties $\operatorname{Rep}\left(\overline{\mathbf{Q}}_{\infty}, \mathbf{d}\right) \cong T^{*} \operatorname{Rep}\left(\mathbf{Q}_{\infty}, \mathbf{d}\right)$, through which we can equip $\operatorname{Rep}\left(\overline{\mathbf{Q}}_{\infty}, \mathbf{d}\right)$ with a symplectic structure. 


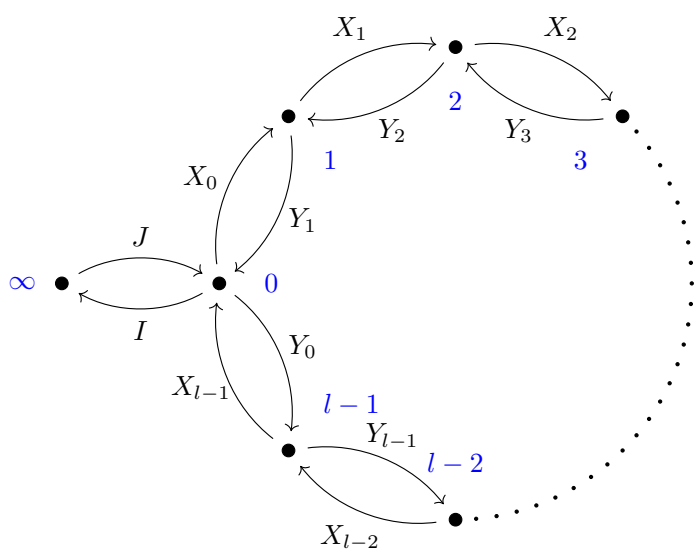

The algebraic group $G(\mathbf{d}):=\prod_{i=0}^{l-1} \mathrm{GL}\left(\mathbf{V}_{i}\right)$ acts on $\operatorname{Rep}\left(\overline{\mathbf{Q}}_{\infty}, \mathbf{d}\right)$ by change of basis. If $\mathbf{g}=\left(g_{0}, \ldots, g_{l-1}\right) \in G(\mathbf{d})$ and $(\mathbf{X}, \mathbf{Y}, I, J) \in \operatorname{Rep}\left(\overline{\mathbf{Q}}_{\infty}, \mathbf{d}\right)$ then

$$
\text { g. }(\mathbf{X}, \mathbf{Y}, I, J)=\left(g_{1} X_{0} g_{0}^{-1}, \ldots, g_{0} X_{l-1} g_{l-1}^{-1}, g_{l-1} Y_{0} g_{0}^{-1}, \ldots, g_{l-2} Y_{l-1} g_{l-1}^{-1}, I g_{0}^{-1}, g_{0} J\right) \text {. }
$$

The action of $G(\mathbf{d})$ on $\operatorname{Rep}\left(\overline{\mathbf{Q}}_{\infty}, \mathbf{d}\right)$ is Hamiltonian. The moment map for this action is given by

$$
\mu_{\mathbf{d}}: \operatorname{Rep}\left(\overline{\mathbf{Q}}_{\infty}, \mathbf{d}\right) \rightarrow \mathfrak{g}(\mathbf{d})^{*} \cong \mathfrak{g}(\mathbf{d}):=\operatorname{Lie} G(\mathbf{d}), \quad(\mathbf{X}, \mathbf{Y}, I, J) \mapsto[\mathbf{X}, \mathbf{Y}]+J I .
$$

3.2. Quiver varieties. If $\mathbf{d} \in\left(\mathbb{Z}_{\geq 0}\right)^{l}$ and $\theta=\left(\theta_{0}, \ldots, \theta_{l-1}\right) \in \mathbb{Q}^{l}$, we will also write $\theta=\left(\theta_{0} \operatorname{id}_{0}, \theta_{1} \operatorname{id}_{1}, \ldots, \theta_{l-1} \operatorname{id}_{l-1}\right) \in \mathfrak{g}(\mathbf{d})$, where $\operatorname{id}_{i}=\operatorname{id}_{\mathbf{V}_{i}}(i=0, \ldots, l-1)$. Define

$$
\mathcal{X}_{\theta}(\mathbf{d}):=\mu_{\mathbf{d}}^{-1}(\theta) / / G(\mathbf{d}):=\operatorname{Spec} \mathbb{C}\left[\mu_{\mathbf{d}}^{-1}(\theta)\right]^{G(\mathbf{d})} \text {. }
$$

We will always assume that the parameter $\theta$ is chosen in such a way that the variety $\mathcal{X}_{\theta}(\mathbf{d})$ is smooth. Moreover, define the GIT quotient

$$
\mathcal{M}_{\theta}(\mathbf{d}):=\mu_{\mathbf{d}}^{-1}(0) / /{ }_{\theta} G(\mathbf{d})=\operatorname{Proj} \bigoplus_{i \geq 0} \mathbb{C}\left[\mu_{\mathbf{d}}^{-1}(0)\right]^{\chi_{\theta}^{i}}
$$

where $\chi_{\theta}: G(\mathbf{d}) \rightarrow \mathbb{C}^{*}$ is the character sending $\mathbf{g}$ to $\prod\left(\operatorname{det} g_{i}\right)^{\theta_{i}}$ and $\mathbb{C}\left[\mu_{\mathbf{d}}^{-1}(0)\right]^{\chi_{\theta}^{i}}$ denotes the space of semi-invariant functions on $\mu_{\mathbf{d}}^{-1}(0)$, i.e., those functions $f$ satisfying $\mathbf{g} . f=$ $\chi_{\theta}^{i}(\mathbf{g}) f$. By definition, the space $\mathbb{C}\left[\mu_{\mathbf{d}}^{-1}(0)\right]^{\chi_{\theta}^{i}}$ is zero unless $i \theta \in \mathbb{Z}^{l}$.

The varieties $\mathcal{X}_{\theta}(\mathbf{d})$ and $\mathcal{M}_{\theta}(\mathbf{d})$ can be endowed with hyper-Kähler structures (see e.g. [16, §3.6]). Moreover, the group $\mathbb{C}^{*}$ acts on $\operatorname{Rep}\left(\overline{\mathbf{Q}}_{\infty}, \mathbf{d}\right)$ by the rule $t .(\mathbf{X}, \mathbf{Y}, I, J)=$ $\left(t^{-1} \mathbf{X}, t \mathbf{Y}, I, J\right)$ for $t \in \mathbb{C}^{*}$. This action descends to actions on $\mathcal{X}_{\theta}(\mathbf{d})$ and $\mathcal{M}_{\theta}(\mathbf{d})$. By $\mathcal{X}_{\theta}(\mathbf{d})^{\mathbb{C}^{*}}$ and $\mathcal{M}_{\theta}(\mathbf{d})^{\mathbb{C}^{*}}$ we will always mean the sets of closed $\mathbb{C}^{*}$-fixed points.

Let us recall the definition of the tautological bundle on a quiver variety. Assume that the group $G(\mathbf{d})$ acts freely on the fibre $\mu_{\mathbf{d}}^{-1}(\theta)$ and consider the trivial vector bundle $\widehat{\mathcal{V}}_{\theta}(\mathbf{d}):=\mu_{\mathbf{d}}^{-1}(\theta) \times \widehat{\mathbf{V}}$ on $\mu_{\mathbf{d}}^{-1}(\theta)$. We regard $\widehat{\mathcal{V}}_{\theta}(\mathbf{d})$ as a $\mathbb{C}^{*}$-equivariant vector bundle by letting $\mathbb{C}^{*}$ act trivially on $\widehat{\mathbf{V}}$. Let $G(\mathbf{d})$ act diagonally on $\widehat{\mathcal{V}}_{\theta}(\mathbf{d})$. The vector bundle $\widehat{\mathcal{V}}_{\theta}(\mathbf{d})$ descends to a $\mathbb{C}^{*}$-equivariant vector bundle

$$
\mathcal{V}_{\theta}(\mathbf{d}):=\mu_{\mathbf{d}}^{-1}(\theta) \times{ }^{G(\mathbf{d})} \widehat{\mathbf{V}}=\left(\mu_{\mathbf{d}}^{-1}(\theta) \times \widehat{\mathbf{V}}\right) / / G(\mathbf{d})
$$

on $\mathcal{X}_{\theta}(\mathbf{d})$, which is called the tautological bundle.

Notation. We will always consider the subscript $i$ in the expressions $d_{i}, \mathbf{V}_{i}, g_{i}, X_{i}, Y_{i}, \theta_{i}$ modulo $l$ (unless $i=\infty$ ).

3.3. The Etingof-Ginzburg isomorphism. Throughout this subsection we assume $\mathbf{d}=n \delta$, where $\delta:=(1, \ldots, 1) \in \mathbb{Z}^{l}$. Given $\mathbf{h}$ as in Definition 2.2, set

$$
\theta_{\mathbf{h}}=\left(\theta_{0}, \ldots, \theta_{l-1}\right)=\left(-h+H_{0}, H_{1}, \ldots, H_{l-1}\right) .
$$

Since we are assuming that the parameter $\mathbf{h}$ is generic, the group $G(n \delta)$ acts freely on the fibre $\mu_{n \delta}^{-1}\left(\theta_{\mathbf{h}}\right)$ (see [12, Proposition 11.11]). We abbreviate

$$
\mathcal{C}_{\mathbf{h}}:=\mathcal{X}_{\theta_{\mathbf{h}}}(n \delta), \quad \mathcal{V}_{\mathbf{h}}:=\mathcal{V}_{\theta_{\mathbf{h}}}(n \delta)
$$


Consider $\left(\mathbb{C} \Gamma_{n}\right)^{\Gamma_{n-1}}$ as a module over $\left\langle\epsilon_{1}\right\rangle=\mathbb{Z} / l \mathbb{Z}$. It decomposes as a direct sum of $n$-dimensional isotypic components $\left(\mathbb{C} \Gamma_{n}\right)_{\chi_{i}}^{\Gamma_{n-1}}$, where $\chi_{i}$ is the character $\chi_{i}: \epsilon_{1} \mapsto \eta^{i}$. Fix a linear isomorphism

$$
\left(\mathbb{C} \Gamma_{n}\right)^{\Gamma_{n-1}} \rightarrow \widehat{\mathbf{V}}
$$

mapping each $\left(\mathbb{C} \Gamma_{n}\right)_{\chi_{i}}^{\Gamma_{n-1}}$ onto $\mathbf{V}_{i}$. It induces an isomorphism of endomorphism algebras

$$
\varpi: \operatorname{End}_{\mathbb{C}}\left(\left(\mathbb{C}_{n}\right)^{\Gamma_{n-1}}\right) \rightarrow \operatorname{End}_{\mathbb{C}}(\widehat{\mathbf{V}}) .
$$

Definition 3.1. Recall $\operatorname{Rep}_{\mathbb{C}_{n}}\left(\mathbb{H}_{\mathbf{h}}\right)$ from Definition 2.6. Each $\phi \in \operatorname{Rep}_{\mathbb{C}_{n}}\left(\mathbb{H}_{\mathbf{h}}\right)$ defines endomorphisms $\phi\left(x_{1}\right), \phi\left(y_{1}\right): \mathbb{C} \Gamma_{n} \rightarrow \mathbb{C} \Gamma_{n}$, where $x_{1}, y_{1} \in \mathbb{H}_{\mathbf{h}}$ are as in Definition 2.2. Set

$$
\mathbf{X}(\phi):=\varpi\left(\left.\phi\left(x_{1}\right)\right|_{\left(\mathbb{C} \Gamma_{n}\right)^{\Gamma_{n-1}}}\right), \quad \mathbf{Y}(\phi):=\varpi\left(\left.\phi\left(y_{1}\right)\right|_{\left(\mathbb{C} \Gamma_{n}\right)^{\Gamma_{n-1}}}\right) .
$$

Consider the maps

$$
\begin{aligned}
\Psi: \operatorname{Rep}_{\mathbb{C}_{n}}^{o}\left(\mathbb{H}_{\mathbf{h}}\right) \rightarrow \operatorname{Rep}(\overline{\mathbf{Q}}, n \delta), & \phi \mapsto(\mathbf{X}(\phi), \mathbf{Y}(\phi)), \\
p: \operatorname{Rep}\left(\overline{\mathbf{Q}}_{\infty}, n \delta\right) \rightarrow \operatorname{Rep}(\overline{\mathbf{Q}}, n \delta), & (\mathbf{X}, \mathbf{Y}, I, J) \mapsto(\mathbf{X}, \mathbf{Y})
\end{aligned}
$$

Lemma 3.2. The maps $\Psi$ and $p$ are $\mathbb{C}^{*}$-equivariant.

Proof. The equivariance of $p$ is obvious. Let $t \in \mathbb{C}^{*}, \phi \in \operatorname{Rep}_{\mathbb{C}_{n}}\left(\mathbb{H}_{\mathbf{h}}\right)$ and $z \in \mathbb{H}_{\mathbf{h}}$. We have $(t . \phi)(z)=\phi\left(t^{-1} . z\right)$, so $(t . \phi)\left(x_{1}\right)=t^{-1} \phi\left(x_{1}\right)$ and $(t . \phi)\left(y_{1}\right)=t \phi\left(y_{1}\right)$. Hence

$$
\begin{aligned}
\Psi(t . \phi) & =\left(\varpi\left(\left.t^{-1} \phi\left(x_{1}\right)\right|_{\left(\mathbb{C \Gamma}_{n}\right)^{\Gamma_{n-1}}}\right), \varpi\left(\left.t \phi\left(y_{1}\right)\right|_{\left(\mathbb{C}_{n}\right)^{\Gamma_{n-1}}}\right)\right) \\
& =\left(t^{-1} \mathbf{X}(\phi), t \mathbf{Y}(\phi)\right)=t .(\mathbf{X}(\phi), \mathbf{Y}(\phi)) .
\end{aligned}
$$

The proof of [12, Proposition 11.24] carries over directly to yield the following generalization.

Theorem 3.3. Maps (12) and $(13)$ induce a $\mathbb{C}^{*}$-equivariant isomorphism of varieties

$$
\mathrm{EG}: \mathcal{Y}_{\mathbf{h}} \stackrel{\sim}{\rightarrow} \mathcal{C}_{\mathbf{h}}
$$

and vector bundles

$$
\mathcal{R}_{\mathbf{h}}^{\Gamma_{n-1}} \stackrel{\sim}{\rightarrow} \mathcal{V}_{\mathbf{h}}
$$

We call (14) the Etingof-Ginzburg isomorphism.

\section{Combinatorics}

We recall several combinatorial notions which will be used throughout the paper.

4.1. Young diagrams. If $\mu$ is a partition of $k$, set $n(\mu):=\sum_{i \geq 1} i \cdot \mu_{i+1}$. If $\underline{\lambda}$ is an $l$-multipartition of $k$, define $r(\underline{\lambda})=\sum_{i=1}^{l-1} i \cdot\left|\lambda^{i}\right|$. Recall the notations $[n]_{t}=\frac{1-t^{n}}{1-t}=$ $1+\ldots+t^{n-1}$ and $(t)_{n}=(1-t)\left(1-t^{2}\right) \ldots\left(1-t^{n}\right)$.

Let $\mu=\left(\mu_{1}, \ldots, \mu_{m}, 0, \ldots\right)$ be a partition of $k$, where $\mu_{1}, \ldots, \mu_{m}$ are non-zero. Let $\mathbb{Y}(\mu):=\left\{(i, j) \mid 1 \leq i \leq m, 1 \leq j \leq \lambda_{i}\right\}$ denote the Young diagram of $\mu$. We will always display Young diagrams according to the English convention. We call each pair $(i, j) \in \mathbb{Y}(\mu)$ a cell. We will often use the symbol $\square$ to refer to cells. Sometimes we will also abuse notation and write $\mu$ instead of $\mathbb{Y}(\mu)$ where no confusion can arise, e.g., $\square \in \mu$ instead of $\square \in \mathbb{Y}(\mu)$. If $\square=(i, j) \in \mathbb{Y}(\mu)$ is a cell, let $c(\square):=j-i$ be the content of $\square$. We call $\operatorname{Res}_{\mu}(t):=\sum_{\square \in \mu} t^{c(\square)}$ the residue of $\mu$. We also call $c(\square) \bmod l$ the $l$-content of $\square$ and $\sum_{\square \in \mu} t^{c(\square) \bmod l}$ the l-residue of $\mu$. It is clear that a partition is determined uniquely by its residue.

Now suppose that $\underline{\lambda}$ is an $l$-multipartition of $k$. By the Young diagram of $\underline{\lambda}$ we mean the $l$-tuple $\mathbb{Y}(\underline{\lambda}):=\left(\mathbb{Y}\left(\lambda^{0}\right), \ldots, \mathbb{Y}\left(\lambda^{l-1}\right)\right)$. By a cell $\square \in \mathbb{Y}(\underline{\lambda})$ we mean a cell in any of the Young diagrams $\mathbb{Y}\left(\lambda^{i}\right)$. Let $\mathbf{e}=\left(e_{0}, \ldots, e_{l-1}\right) \in \mathbb{Q}^{l}$. We define the e-residue of $\underline{\lambda}$ to be

$$
\operatorname{Res}_{\underline{\underline{\lambda}}}^{\mathbf{e}}(t):=\sum_{i=0}^{l-1} t^{e_{i}} \operatorname{Res}_{\lambda^{i}}(t) \text {. }
$$

For sufficiently generic $\mathbf{e}$, an $l$-multipartition is determined uniquely by its e-residue. 
4.2. Hook length polynomials. Let $\mu$ be a partition and fix a cell $(i, j) \in \mathbb{Y}(\mu)$. By the hook associated to the cell $(i, j)$ we mean the set $\{(i, j)\} \cup\left\{\left(i^{\prime}, j\right) \in \mathbb{Y}(\mu) \mid i^{\prime}>\right.$ $i\} \cup\left\{\left(i, j^{\prime}\right) \in \mathbb{Y}(\mu) \mid j^{\prime}>j\right\}$. We call $(i, j)$ the root of the hook, $\left\{\left(i^{\prime}, j\right) \in \mathbb{Y}(\mu) \mid i^{\prime}>i\right\}$ the leg of the hook and $\left\{\left(i, j^{\prime}\right) \in \mathbb{Y}(\mu) \mid j^{\prime}>j\right\}$ the arm of the hook. The cell in the leg of the hook with the largest first coordinate is called the foot of the hook, and the cell in the arm of the hook with the largest second coordinate is called the hand of the hook.

By a hook in $\mathbb{Y}(\mu)$ we mean a hook associated to some cell $\square \in \mathbb{Y}(\mu)$. If $H$ is a hook, let $\operatorname{arm}(H)$ denote its arm and let $\operatorname{leg}(H)$ denote its leg. If $\square$ is the root of $H$, let $a_{\mu}(\square):=|\operatorname{arm}(H)|$ and $l_{\mu}(\square):=|\operatorname{leg}(H)|$. Set $h_{\mu}(\square):=1+a_{\mu}(\square)+l_{\mu}(\square)$. The hook length polynomial of the partition $\mu$ is

$$
H_{\mu}(t):=\prod_{\square \in \mu}\left(1-t^{h_{\mu}(\square)}\right) .
$$

Hook length polynomials are related to Schur functions by the following equality

$$
s_{\mu}\left(1, t, t^{2}, \ldots\right)=\frac{t^{n(\mu)}}{H_{\mu}(t)} .
$$

4.3. Frobenius form of a partition. By a Frobenius hook in $\mathbb{Y}(\mu)$ we mean a hook whose root is a cell of content zero. Clearly $\mathbb{Y}(\mu)$ is the disjoint union of all its Frobenius hooks. Suppose that $(1,1),(2,2), \ldots,(k, k)$ are the cells of content zero in $\mathbb{Y}(\mu)$. Let $F_{i}$ denote the Frobenius hook with root $(i, i)$. We endow the set of Frobenius hooks with the natural ordering $F_{1}<F_{2}<\ldots<F_{k}$. We call $F_{1}$ the innermost or first Frobenius hook and $F_{k}$ the outermost or last Frobenius hook. Let $a_{i}=a_{\mu}(i, i)$ and $b_{i}=l_{\mu}(i, i)$. We call $\left(a_{1}, \ldots, a_{k} \mid b_{1}, \ldots, b_{k}\right)$ the Frobenius form of $\mu$.

4.4. Bead diagrams. Let us recall the notion of a bead diagram (see e.g. [21, §2.7]). We call an element $(i, j)$ of $\mathbb{Z}_{\leq-1} \times\{0, \ldots, l-1\}$ a point. We say that the point $(i, j)$ lies to the left of $\left(i, j^{\prime}\right)$ if $j<j^{\prime}$, and that $(i, j)$ lies above $\left(i^{\prime}, j\right)$ if $i^{\prime}<i$.

A bead diagram is a function $f: \mathbb{Z}_{\leq-1} \times\{0, \ldots, l-1\} \rightarrow\{0,1\}$ which takes value 1 for only finitely many points. If $f(i, j)=1$ we say that the point $(i, j)$ is occupied by a bead. If $f(i, j)=0$ we say that the point $(i, j)$ is empty. Suppose that a point $(i, j)$ is empty and that there exists an $i^{\prime}<i$ such that the point $\left(i^{\prime}, j\right)$ is occupied by a bead. Then we call the point $(i, j)$ a gap.

We say that a point $(i, j) \in \mathbb{Z}_{\leq-1} \times\{0, \ldots, l-1\}$ is in the $(-i)$-th row and $j$-th column (or runner) of the bead diagram. We call the $i$-th row full (empty) if every point $(i, k)$ for $k=0, \ldots, l-1$ is occupied by a bead (is empty). A row is called redundant if it is a full row and if all the rows above it are full. A graphical interpretation of the notion of a bead diagram can be found in Example 4.6. We only display the rows containing at least one bead or gap.

Definition 4.1. Let $\mu \in \mathcal{P}$ and $p \geq \ell(\mu)$. Set

$$
\beta_{i}^{p}=\mu_{i}+p-i \quad(1 \leq i \leq p) .
$$

We call $\left\{\beta_{i}^{p} \mid 1 \leq i \leq p\right\}$ a set of $\beta$-numbers for $\mu$. Note that $\left|\left\{\beta_{i}^{p} \mid 1 \leq i \leq p\right\}\right|=p$. From each set of $\beta$-numbers one can uniquely recover the corresponding partition $\mu$.

Definition 4.2. Given a set of $\beta$-numbers $\left\{\beta_{i}^{p} \mid 1 \leq i \leq p\right\}$ we can naturally associate to it a bead diagram by the rule

$$
f(i, j)=1 \Longleftrightarrow-(i+1) \cdot l+j \in\left\{\beta_{i}^{p} \mid 1 \leq i \leq p\right\} .
$$

Let $\mu$ be as in Definition 4.1. If $p$ is the smallest multiple of $l$ satisfying $p \geq \ell(\mu)$ we denote the resulting bead diagram by $\mathbb{B}(\mu)$. The diagram $\mathbb{B}(\mu)$ has no redundant rows and the number of beads in $\mathbb{B}(\mu)$ is a multiple of $l$.

Remark 4.3. Conversely, if we are given a bead diagram $f$, the set $\{-(i+1) \cdot l+j \mid$ $f(i, j)=1\}$ is a set of $\beta$-numbers for some partition. The relationship between bead diagrams, sets of $\beta$-numbers and partitions can therefore be illustrated as follows

$$
\{\text { bead diagrams }\} \longleftrightarrow \text { sets of } \beta \text {-numbers }\} \rightarrow \text { \{partitions }\},
$$

where the set of partitions contains partitions of an arbitrary integer. 
4.5. Cores and quotients. Let $f: \mathbb{Z}_{<-1} \times\{0, \ldots, l-1\} \rightarrow\{0,1\}$ be a bead diagram. Suppose that the point $(i, j)$ with $i<-1$ is occupied by a bead, i.e., $f(i, j)=1$, and that $f(i+1, j)=0$. To slide or move the bead in position $(i, j)$ upward means to modify the function $f$ by setting $f^{\prime}(i, j)=0, f^{\prime}(i+1, j)=1$ and $f^{\prime}=f$ otherwise.

Definition 4.4. Let $\mu$ be a partition. Take any bead diagram $f$ corresponding to $\mu$. We obtain a new bead diagram $f^{\prime}$ by sliding beads upward as long as it is possible. We call the partition corresponding to the bead diagram $f^{\prime}$ the $l$-core of $\mu$, denoted Core $(\mu)$. Let $\mathcal{Q}(l)$ denote the set of all $l$-cores. We set

$$
\mathcal{P}_{\nu}(k):=\{\mu \in \mathcal{P}(k) \mid \operatorname{Core}(\mu)=\nu\} .
$$

Definition 4.5. Consider the bead diagram $\mathbb{B}(\mu)$. Each column of $\mathbb{B}(\mu)$ can itself be considered as a bead diagram for $l=1$. Let $Q^{i}(\mu)$ denote the partition corresponding to the $i$-th column. We call the $l$-multipartition Quot $(\mu):=\left(Q^{0}(\mu), Q^{1}(\mu), \ldots, Q^{l-1}(\mu)\right)$ the l-quotient of $\mu$.

A partition is determined uniquely by its $l$-core and $l$-quotient [21, Theorem 2.7.30]. In particular, there is a bijection

$$
\mathcal{P}_{\varnothing}(n l) \rightarrow \mathcal{P}(l, n), \quad \mu \mapsto \underline{\text { Quot }}(\mu) .
$$

Example 4.6. Consider the partition $\mu=(6,5,3,3,1,1)$ and take $l=3$. The first-column hook-lengths are $11,9,6,5,2,1$. They form a set of $\beta$-numbers. The bead diagram below on the left illustrates $\mathbb{B}(\mu)$ while the diagram on the right illustrates the effect of sliding all the beads upward.

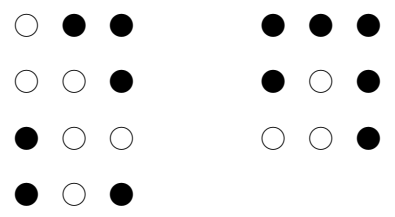

Let us read off the 3-core of $\mu$ from the diagram on the right. We can ignore all the beads before the first empty point, which we label as zero. We carry on counting. The remaining two beads get labels 1 and 4 . These form a set of $\beta$-numbers, which we can interpret as the first-column hook-lengths corresponding to the partition $(3,1)$. It follows that $\operatorname{Core}(\mu)=(3,1)$. To determine the 3 -quotient of $\mu$ we divide the diagram on the left into three columns and consider each separately. We read off the $\beta$-numbers as before they are 2, 3 for the first column, 0 for the second column and 1 for the third column. It follows that $\underline{\operatorname{Quot}}(\mu)=((2,2), \varnothing,(1))$.

4.6. Rim-hooks. The rim of $\mathbb{Y}(\mu)$ is the subset of $\mathbb{Y}(\mu)$ consisting of the cells $(i, j)$ such that $(i+1, j+1)$ does not lie in $\mathbb{Y}(\mu)$. Fix a cell $(i, j) \in \mathbb{Y}(\mu)$. Recall that by the hook associated to $(i, j)$ we mean the subset of $\mathbb{Y}(\mu)$ consisting of all the cells $(i, k)$ with $k \geq j$ and all the cells $(k, j)$ with $k \geq i$. We define the rim-hook associated to the cell $(i, j)$ to be the intersection of the set $\left\{\left(i^{\prime}, j^{\prime}\right) \mid i^{\prime} \geq i, j^{\prime} \geq j\right\}$ with the rim of $\mathbb{Y}(\mu)$. We call a rim-hook an $l$-rim-hook if it contains $l$ cells. The $l$-core of $\mu$ can also be characterised as the subpartition $\mu^{\prime}$ of $\mu$ obtained from $\mu$ by a successive removal of $l$-rim-hooks, in whichever order (see [21, Theorem 2.7.16]).

Lemma 4.7 (21, Lemma 2.7.13]). Let $R$ be an l-rim-hook in $\mu$ and set $\mu^{\prime}:=\mu-R$. Then $\underline{\operatorname{Quot}}\left(\mu^{\prime}\right)=\underline{\mathrm{Quot}}(\mu)-\square$ for some $\square \in \underline{\mathrm{Quot}}(\mu)$.

4.7. The $\tilde{S}_{l}$-action on partitions. Assume for the rest of this section that $l>1$. All subscripts should be regarded modulo $l$. Let $\tilde{S}_{l}$ denote the affine symmetric group. It has a Coxeter presentation with generators $\sigma_{0}, \ldots, \sigma_{l-1}$ and relations

$$
\sigma_{i}^{2}=1, \quad \sigma_{i} \sigma_{i+1} \sigma_{i}=\sigma_{i+1} \sigma_{i} \sigma_{i+1} \quad(0 \leq i \leq l-1) .
$$

Let us recall the $\tilde{S}_{l}$-action on the set $\mathcal{P}$ of all partitions from [25, §4]. We will later use this action to describe the behaviour of the $\mathbb{C}^{*}$-fixed points under reflection functors. We need the following definition, reminiscent of the combinatorics of the Fock space. 
Definition 4.8. Let $k \in\{0, \ldots, l-1\}$. Consider the Young diagram $\mathbb{Y}(\mu)$ as a subset of the $\mathbb{Z}_{>0} \times \mathbb{Z}_{>0}$ space. We say that a cell $(i, j) \in \mathbb{Y}(\mu)$ is removable if $\mathbb{Y}(\mu)-\{(i, j)\}$ is the Young diagram of a partition. We say that it is $k$-removable if additionally $c(i, j)=$ $j-i=k \bmod l$. We call a cell $(i, j) \notin \mathbb{Y}(\mu)$ addable if $\mathbb{Y}(\mu) \cup\{(i, j)\}$ is the Young diagram of a partition. We call it $k$-addable if additionally $c(i, j)=j-i=k \bmod l$.

We discuss the combinatorics of removability and addability in more detail in $\$ 9.5$. We will, in particular, require Lemma 9.10 proven there.

Definition 4.9. Suppose that $\mu \in \mathcal{P}$ and $k \in\{0, \ldots, l-1\}$. Define $\mathbf{T}_{k}(\mu)$ to be the partition such that

$$
\mathbb{Y}\left(\mathbf{T}_{k}(\mu)\right)=\mathbb{Y}(\mu) \cup\{\square \text { is } k \text {-addable }\}-\{\square \text { is } k \text {-removable }\} .
$$

The group $\tilde{S}_{l}$ acts on $\mathcal{P}$ by the rule

$$
\sigma_{i} * \mu=\mathbf{T}_{i}(\mu) \quad(\mu \in \mathcal{P}, i \in \mathbb{Z} / l \mathbb{Z}) .
$$

This action also plays a role in the combinatorics of the Schubert calculus of the affine Grassmannian, see [23, §8.2] and [24, §11]. By [24, Proposition 22], we have $\tilde{S}_{l} * \varnothing=\varnothing(l)$. Let us recall how the $\tilde{S}_{l}$-action behaves with respect to cores and quotients. Consider the finite symmetric group $S_{l}$ as the group of permutations of the set $\{0, \ldots, l-1\}$. Let $s_{i} \in S_{l}$ $(i=1, \ldots, l-1)$ be the transposition swapping $i-1$ and $i$. Let $s_{0}$ be the transposition swapping 0 and $l-1$. Note that our conventions for $S_{l}$ differ from those for $S_{n}$ introduced in $\$ 2.2$ The finite symmetric group $S_{l}$ acts on the set $\underline{\mathcal{P}}$ of all $l$-multipartitions by the rule

$$
w \cdot \underline{\lambda}=\left(\lambda^{w^{-1}(0)}, \ldots, \lambda^{w^{-1}(l-1)}\right), \quad w \in S_{l} .
$$

Consider the group homomorphism

$$
\operatorname{pr}: \tilde{S}_{l} \rightarrow S_{l}, \quad \sigma_{i} \mapsto s_{i}(i=0, \ldots, l-1) .
$$

Proposition 4.10 ([25, Proposition 4.13]). Let $\mu \in \mathcal{P}$ and $\sigma \in \tilde{S}_{l}$. Then

$$
\operatorname{Core}(\sigma * \mu)=\sigma * \operatorname{Core}(\mu), \quad \underline{\operatorname{Quot}}(\sigma * \mu)=\operatorname{pr}(\sigma) \cdot \underline{\operatorname{Quot}}(\mu) .
$$

4.8. Partitions and the cyclic quiver. Let $N_{i}(\lambda)$ be the number of cells of $l$-content $i$ in $\mathbb{Y}(\lambda)$. Using this notation, the $l$-residue of $\lambda$ equals $\sum_{i=0}^{l-1} N_{i}(\lambda) t^{i}$. Consider the map

$$
\mathfrak{d}: \mathcal{P} \rightarrow \mathbb{Z}^{l}, \quad \lambda \mapsto \mathbf{d}_{\lambda}:=\left(N_{0}(\lambda), \ldots, N_{l-1}(\lambda)\right) .
$$

We interpret this map as assigning to every partition a dimension vector for the cyclic quiver with $l$ vertices. Let

$$
\mathbb{Z}_{\odot}=\left\{\mathbf{d} \in\left(\mathbb{Z}_{\geq 0}\right)^{l} \mid \mathbf{d}=\mathbf{d}_{\nu} \text { for some } \nu \in \varnothing(l)\right\}
$$

be the set of all dimension vectors corresponding to l-cores. By [21, Theorem 2.7.41] an $l$-core is determined uniquely by its $l$-residue. Hence 17 restricts to a bijection

$$
\mathfrak{d}: \varnothing(l) \longleftrightarrow \mathbb{Z}_{\odot}, \quad \nu \mapsto \mathbf{d}_{\nu} .
$$

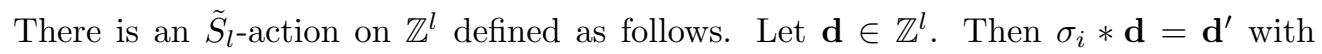
$d_{j}^{\prime}=d_{j}(j \neq i)$ and

$$
d_{i}^{\prime}=d_{i+1}+d_{i-1}-d_{i} \quad(i \neq 0), \quad d_{0}^{\prime}=d_{1}+d_{l-1}-d_{0}+1 \quad(i=0) .
$$

The following proposition follows by an elementary calculation from Lemma 9.10 .

Proposition 4.11. The following diagram is $\tilde{S}_{l}$-equivariant

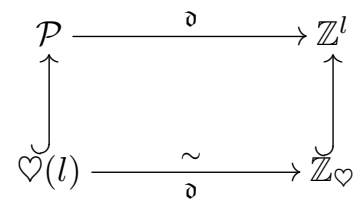

Let $\sigma_{i} \in \tilde{S}_{l}$ and $\nu \in \varnothing(l)$. Then $\sigma_{i} *\left(n \delta+\mathbf{d}_{\nu}\right)=n \delta+\sigma_{i} * \mathbf{d}_{\nu}$ and $\sigma_{i} * \mathbf{d}_{\nu}=\mathfrak{d}\left(\sigma_{i} * \nu\right)$. By [21, Theorem 2.7.41] any partition $\lambda$ of $n l+\left|\sigma_{i} * \nu\right|$ such that $\mathfrak{d}(\lambda)=n \delta+\sigma_{i} * \mathbf{d}_{\nu}$ has $l$-core $\sigma_{i} * \nu$. Hence

$$
\mathcal{P}_{\sigma_{i} * \nu}\left(n l+\left|\sigma_{i} * \nu\right|\right)=\mathfrak{d}^{-1}\left(n \delta+\sigma_{i} * \mathbf{d}_{\nu}\right) .
$$


4.9. Reflection functors. The group $\tilde{S}_{l}$ also acts on the parameter space $\mathbb{Q}^{l}$ for the quiver $\overline{\mathbf{Q}}_{\infty}$ by the rule $\sigma_{i} \cdot \theta=\theta^{\prime}$ with

$$
\theta_{i}^{\prime}=-\theta_{i}, \quad \theta_{i-1}^{\prime}=\theta_{i-1}+\theta_{i}, \quad \theta_{i+1}^{\prime}=\theta_{i+1}+\theta_{i}, \quad \theta_{j}^{\prime}=\theta_{j} \quad(j \notin\{i-1, i, i+1\})
$$

Fix $i \in\{0, \ldots, l-1\}$. Let $\theta \in \mathbb{Q}^{l}$ be such that $\theta_{i} \neq 0$. Choose $\nu \in \oslash(l)$. Let

$$
\mathfrak{R}_{i}: \mathcal{X}_{\theta}\left(n \delta+\mathbf{d}_{\nu}\right) \rightarrow \mathcal{X}_{\sigma_{i} \cdot \theta}\left(n \delta+\sigma_{i} * \mathbf{d}_{\nu}\right)
$$

be the reflection functor associated to the simple reflection $\sigma_{i} \in \tilde{S}_{l}$. These functors were defined by Nakajima [30, §3] and Crawley-Boevey and Holland [7, §2], [8, §5]. One can endow the varieties $\mathcal{X}_{\theta}\left(n \delta+\mathbf{d}_{\nu}\right), \mathcal{X}_{\sigma_{i} \cdot \theta}\left(n \delta+\sigma_{i} * \mathbf{d}_{\nu}\right)$ with hyper-Kähler structures with respect to which the reflection functor $\mathfrak{R}_{i}$ is a $U(1)$-equivariant hyper-Kähler isometry.

\section{5. $\mathbb{C}^{*}$-FIXED POINTS IN QUIVER VARIETIES}

In this section we explicitly construct the $\mathbb{C}^{*}$-fixed points in the quiver varieties $\mathcal{X}_{\theta}(n \delta+$ $\mathbf{d}_{\nu}$ ), assuming smoothness, as conjugacy classes of quadruples of certain matrices. Our description generalizes the work of Wilson, who classified the $\mathbb{C}^{*}$-fixed points in the special case $l=1$ in [36, Proposition 6.11]. Our construction depends on the Frobenius form of a partition. In 85.1 we define the matrices representing the fixed points in the special case when a partition consists of a single Frobenius hook. In $\$ 5.2$ we define more general matrices for arbitrary partitions. In 5.3 we interpret our matrices as quiver representations and show that the corresponding orbits are in fact fixed under the $\mathbb{C}^{*}$-action. We finish by computing the character of the fibre of the tautological bundle at each fixed point.

5.1. The matrix $A(m, r)$. Fix $\theta \in \mathbb{Q}^{l}$. The subscript in $\theta_{i}$ should always be considered modulo $l$. Suppose that $M$ is a matrix. Let $M_{i j}$ denote the entry of $M$ in the $i$-th row and $j$-th column.

Definition 5.1. Let $m \geq 1$ and $1 \leq r \leq m$. We let $\Lambda(m)$ denote the $m \times m$ matrix with 1 's on the first diagonal and all other entries equal to 0 . Let $A(m, r)$ denote the $m \times m$ matrix whose only nonzero entries lie on the $(-1)$-st diagonal and satisfy

$$
A(m, r)_{j+1, j}=\left\{\begin{array}{ccc}
\sum_{i=1}^{j} \theta_{r-i} & \text { if } & 1 \leq j<r \\
-\sum_{i=0}^{m-j-1} \theta_{-m+r+i} & \text { if } & r \leq j \leq m-1 .
\end{array}\right.
$$

Lemma 5.2. The matrix $[\Lambda(m), A(m, r)]$ is diagonal with eigenvalues

$$
[\Lambda(m), A(m, r)]_{j, j}=\left\{\begin{array}{ccc}
\theta_{r-j} & \text { if } & 1 \leq j \neq r \leq m \\
-\sum_{i=1}^{r-1} \theta_{r-i}-\sum_{i=0}^{m-r-1} \theta_{-m+r+i} & \text { if } & j=r .
\end{array}\right.
$$

Proof. Let $\alpha_{j}:=A(m, r)_{j+1, j}$. We have $\Lambda(m) A(m, r)=\operatorname{diag}\left(\alpha_{1}, \alpha_{2}, \ldots, \alpha_{m-1}, 0\right)$ and $A(m, r) \Lambda(m)=\operatorname{diag}\left(0, \alpha_{1}, \alpha_{2}, \ldots, \alpha_{m-1}\right)$. Hence $[\Lambda(m), A(m, r)]=\operatorname{diag}\left(\alpha_{1}, \alpha_{2}-\alpha_{1}, \ldots\right.$, $\left.\alpha_{m-1}-\alpha_{m-2},-\alpha_{m-1}\right)$.

Example 5.3. Let $l=3, m=8, r=5$. Then $A(m, r)$ is the following matrix

$$
\left(\begin{array}{cccccccc}
0 & 0 & 0 & 0 & 0 & 0 & 0 & 0 \\
\theta_{1} & 0 & 0 & 0 & 0 & 0 & 0 & 0 \\
0 & \theta_{1}+\theta_{0} & 0 & 0 & 0 & 0 & 0 & 0 \\
0 & 0 & \theta_{2}+\theta_{1}+\theta_{0} & 0 & 0 & 0 & 0 & 0 \\
0 & 0 & 0 & \theta_{2}+2 \theta_{1}+\theta_{0} & 0 & 0 & 0 & 0 \\
0 & 0 & 0 & 0 & -\theta_{2}-\theta_{1}-\theta_{0} & 0 & 0 & 0 \\
0 & 0 & 0 & 0 & 0 & -\theta_{1}-\theta_{0} & 0 & 0 \\
0 & 0 & 0 & 0 & 0 & 0 & -\theta_{0} & 0
\end{array}\right)
$$


5.2. The matrix $A(\mu)$. Let $\nu \in \mathcal{P}(l)$ and $\mu \in \mathcal{P}_{\nu}(n l+|\nu|)$. Let us write it in Frobenius form $\mu=\left(a_{1}, \ldots, a_{k} \mid b_{1}, \ldots, b_{k}\right)$ (see 4.3 . For each $1 \leq i \leq k$, let $r_{i}=b_{i}+1$, $m_{i}=a_{i}+b_{i}+1$ and $\beta_{i}=\theta_{0}+\sum_{i=1}^{r_{i}-1} \theta_{r_{i}-i}+\sum_{i=0}^{m-r_{i}-1} \theta_{-m+r_{i}+i}$.

Definition 5.4. We define $A(\mu)$ to be the matrix with diagonal blocks $A(\mu)^{i i}=A\left(m_{i}, r_{i}\right)$ and off-diagonal blocks $A(\mu)^{i j}$, where $A(\mu)^{i j}$ is the unique $m_{i} \times m_{j}$ matrix with nonzero entries only on the $\left(r_{j}-r_{i}-1\right)$-th diagonal satisfying

$$
\Lambda\left(m_{i}\right) A(\mu)^{i j}-A(\mu)^{i j} \Lambda\left(m_{j}\right)=-\beta_{i} E\left(r_{i}, r_{j}\right),
$$

where $E\left(r_{i}, r_{j}\right)$ is the $m_{i} \times m_{j}$ matrix with $E\left(r_{i}, r_{j}\right)_{s, t}=0$ unless $s=r_{i}, t=r_{j}$ and $E\left(r_{i}, r_{j}\right)_{r_{i}, r_{j}}=1$.

Explicitly, if $i>j$ then the non-zero diagonal of $A(\mu)^{i j}$ has $r_{i}$ entries equal to $\beta_{i}$ followed by $m_{i}-r_{i}$ entries equal to zero. If $i<j$ then the non-zero diagonal of $A(\mu)^{i j}$ has $r_{j}-1$ entries equal to 0 followed by $n_{j}-r_{j}+1$ entries equal to $-\beta_{i}$.

Example 5.5. Let $l=3$ and $\mu=(3,1 \mid 2,1)$. Then $m_{1}=6, m_{2}=3$ and $r_{1}=3, r_{2}=2$. Set $h=\theta_{2}+\theta_{1}+\theta_{0}$. Then $A(\mu)$ is the matrix

$$
\left(\begin{array}{cccccc|ccc}
0 & 0 & 0 & 0 & 0 & 0 & 0 & 0 & 0 \\
\theta_{2} & 0 & 0 & 0 & 0 & 0 & 0 & 0 & 0 \\
0 & \theta_{2}+\theta_{1} & 0 & 0 & 0 & 0 & 0 & 0 & 0 \\
0 & 0 & -\theta_{2}-\theta_{1}-\theta_{0} & 0 & 0 & 0 & 0 & -2 h & 0 \\
0 & 0 & 0 & -\theta_{1}-\theta_{0} & 0 & 0 & 0 & 0 & -2 h \\
0 & 0 & 0 & 0 & -\theta_{0} & 0 & 0 & 0 & 0 \\
\hline h & 0 & 0 & 0 & 0 & 0 & 0 & 0 & 0 \\
0 & h & 0 & 0 & 0 & 0 & \theta_{1} & 0 & 0 \\
0 & 0 & 0 & 0 & 0 & 0 & 0 & -\theta_{2} & 0
\end{array}\right)
$$

Definition 5.6. Let $\Lambda(\mu)=\bigoplus_{i=1}^{k} \Lambda\left(m_{i}\right)$. Setting $q_{i}=\sum_{s=1}^{i-1} m_{s}+r_{i}$, let $J(\mu)$ be the $n l \times 1$ matrix with entry $\beta_{i}$ in the $q_{i}$-th row (for $1 \leq i \leq k$ ) and all other entries zero. Furthermore, let $I(\mu)$ be the $1 \times n l$ matrix with entry 1 in the $q_{i}$-th column (for $1 \leq i \leq k$ ) and all other entries zero. Finally, we set

$$
\mathbf{A}(\mu):=(\Lambda(\mu), A(\mu), I(\mu), J(\mu)) .
$$

5.3. The fixed points. Let us fix an $l$-core $\nu$ and a parameter $\theta \in \mathbb{Q}^{l}$ such that the variety $\mathcal{X}_{\theta}\left(n \delta+\mathbf{d}_{\tau}\right)$ is smooth, where $\tau=\nu^{t}$. Let $\mathbf{d}_{\tau}:=\left(d_{0}, \ldots, d_{l-1}\right)$ and $\mathbf{d}=n \delta+\mathbf{d}_{\tau}$. Fix a complex vector space $\mathbf{V}_{i}^{\tau}$ of dimension $n+d_{i}$ for each $i=0, \ldots, l-1$. Additionally, let $\mathbf{V}_{\infty}$ be a complex vector space of dimension one. Set $\widehat{\mathbf{V}}^{\tau}=\bigoplus_{i=0}^{l-1} \mathbf{V}_{i}^{\tau}$ and $\mathbf{V}^{\tau}=\widehat{\mathbf{V}}^{\tau} \oplus \mathbf{V}_{\infty}$.

We are now going to interpret $\mathbf{A}(\mu)$ as a quiver representation. With this goal in mind we choose a suitable ordered basis of the vector space $\mathbf{V}^{\tau}$. We show that the endomorphisms of $\mathbf{V}^{\tau}$ defined by $\mathbf{A}(\mu)$ with regard to this basis respect the quiver grading and thus constitute a quiver representation. We next show that this quiver representation lies in the fibre of the moment map at $\theta$. This allows us to conclude that the conjugacy class of $\mathbf{A}(\mu)$ is a point in the quiver variety $\mathcal{X}_{\theta}(\mathbf{d})$. We finish by showing that this point is fixed under the $\mathbb{C}^{*}$-action.

Definition 5.7. Consider the sequence Seq $:=\left(1, \ldots, m_{1}, 1, \ldots, m_{2}, \ldots, 1, \ldots, m_{k}\right)$. We call each increasing subsequence of the form $\left(1, \ldots, m_{i}\right)$ the $p$-th block of Seq and denote it by $\operatorname{Seq}_{p}$. Let $u_{j}$ be the $j$-th element in Seq. Let $\zeta:\{1, \ldots, n l+|\nu|\} \rightarrow\{1, \ldots, k\}$ be the function given by the rule

$$
\zeta(j)=p \Longleftrightarrow u_{j} \in \mathrm{Seq}_{p}
$$

For each $1 \leq j \leq n l+|\nu|$ let

$$
\psi(j)=\left(r_{\zeta(j)}-u_{j}\right) \bmod l .
$$

If $p, p^{\prime} \in \mathbb{N}$, let $\delta\left(p, p^{\prime}\right)=1$ if $p=p^{\prime}$ and $\delta\left(p, p^{\prime}\right)=0$ otherwise. For each $0 \leq i \leq l-1$ and $0 \leq j \leq n l+|\nu|$, let $\omega_{i}(j)$ be defined recursively by the formula

$$
\omega_{i}(0)=0, \quad \omega_{i}(j)=\omega_{i}(j-1)+\delta(\psi(j), i) .
$$

For each $0 \leq i \leq l-1$, fix a basis $\left\{v_{i}^{1}, \ldots, v_{i}^{n+d_{i}}\right\}$ of $\mathbf{V}_{i}^{\tau}$. We define a function

$$
\text { Bas: }\{1, \ldots, n l+|\nu|\} \rightarrow\left\{v_{i}^{e_{i}} \mid 0 \leq i \leq l-1,1 \leq e_{i} \leq n+d_{i}\right\}, \quad j \mapsto v_{\psi(j)}^{\omega_{\psi(j)}(j)} .
$$


We also define a function Cell : $\{1, \ldots, n l+|\nu|\} \rightarrow \mathbb{Y}\left(\mu^{t}\right)$ associating to a natural number $j$ a cell in the Young diagram of $\mu$. We define Cell $(j)$ to be the $u_{j}$-th cell in the $\zeta(j)$-th Frobenius hook of $\mu^{t}$, counting from the hand of the hook, moving to the left towards the root of the hook and then down towards the foot.

Lemma 5.8. The functions Cell and Bas are bijections.

Proof. The fact that Cell is a bijection follows directly from the definitions. Observe that $\psi(j)$ equals the $l$-content of Cell $(j)$. We thus have a commutative diagram

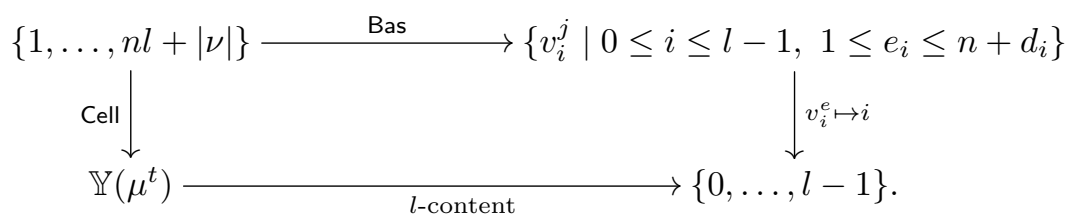

By [21, Theorem 2.7.41], the $l$-residue of $\mu^{t}$ equals $\sum_{i=0}^{l-1}\left(n+d_{i}\right) t^{i}$ because the $l$-core of $\mu^{t}$ is $\tau$. Hence for each $0 \leq i \leq l-1$ there are exactly $n+d_{i}$ elements $j \in\{1, \ldots, n l+|\nu|\}$ such that the $l$-content of Cell $(j)$ equals $i$. By the commutativity of our diagram, we conclude that there are exactly $n+d_{i}$ elements $j \in\{1, \ldots, n l+|\nu|\}$ such that $\operatorname{Bas}(j) \in \mathbf{V}_{i}^{\tau}$.

Now suppose that $j<j^{\prime}$ and $\operatorname{Bas}(j)$, $\operatorname{Bas}\left(j^{\prime}\right) \in \mathbf{V}_{i}^{\tau}$. Then $\psi(j)=\psi\left(j^{\prime}\right)$. Since $j<j^{\prime}$ and the function $\omega_{\psi\left(j^{\prime}\right)}(-)$ is non-decreasing we have $\omega_{\psi\left(j^{\prime}\right)}\left(j^{\prime}\right)=\omega_{\psi\left(j^{\prime}\right)}\left(j^{\prime}-1\right)+1>\omega_{\psi\left(j^{\prime}\right)}\left(j^{\prime}-\right.$ 1) $\geq \omega_{\psi(j)}(j)$. Hence $\operatorname{Bas}(j) \neq \operatorname{Bas}\left(j^{\prime}\right)$. We conclude that the function Bas is injective. Since the domain and codomain have the same cardinality, Bas is also bijective.

Definition 5.9. Let $\mathbb{B}:=(\operatorname{Bas}(1), \operatorname{Bas}(2), \ldots, \operatorname{Bas}(n l+|\tau|))$. By Lemma 5.8, $\mathbb{B}$ is an ordered basis of $\widehat{\mathbf{V}}^{\tau}$. From now on we consider the matrices $\Lambda(\mu)$ and $A(\mu)$ as linear endomorphisms of $\widehat{\mathbf{V}}^{\tau}$ relative to the ordered basis $\mathbb{B}$. Let us choose a nonzero vector $v_{\infty} \in \mathbf{V}_{\infty}$. We consider the matrix $I(\mu)$ as a linear transformation $\widehat{\mathbf{V}}^{\tau} \rightarrow \mathbf{V}_{\infty}$ relative to the ordered bases $\left\{v_{\infty}\right\}$ and $\mathbb{B}$. We also consider the matrix $J(\mu)$ as a linear transformation $\mathbf{V}_{\infty} \rightarrow \widehat{\mathbf{V}}^{\tau}$ relative to the ordered bases $\mathbb{B}$ and $\left\{v_{\infty}\right\}$.

Let $\mu \in \mathcal{P}_{\nu}(n l+|\nu|)$. Suppose that $\mu=\left(a_{1}, \ldots, a_{k} \mid b_{1}, \ldots, b_{k}\right)$ is the Frobenius form of $\mu$. As before, set $r_{i}=b_{i}+1, m_{i}=a_{i}+b_{i}+1$ and $q_{i}=\sum_{j<i} m_{j}+r_{i}$.

Lemma 5.10. Suppose that $1 \leq i \leq k$. Then:

- If $0 \leq j<a_{i}$ then $A(\mu)\left(\operatorname{Bas}\left(q_{i}+j\right)\right)=\sum_{p=1}^{i} c_{p} \operatorname{Bas}\left(q_{p}+j+1\right)$,

- if $0 \leq j=a_{i}$ then $A(\mu)\left(\operatorname{Bas}\left(q_{i}+j\right)\right)=\sum_{p=1}^{i-1} c_{p} \operatorname{Bas}\left(q_{p}+j+1\right)$,

- if $0>j \geq-b_{i}$ then $A(\mu)\left(\operatorname{Bas}\left(q_{i}+j\right)\right)=\sum_{p=1}^{k} c_{p} \mathbf{1}_{-j \leq b_{p}+1} \operatorname{Bas}\left(q_{p}+j+1\right)$

for some coefficients $c_{m} \in \mathbb{C}$, where $\mathbf{1}_{-j \leq b_{p}+1}$ is the indicator function taking value one if $-j \leq b_{p}+1$ and zero otherwise. Moreover, for a generic parameter $\theta$ the coefficients $c_{p}$ are all non-zero.

Proof. This is immediate from Definition 5.4 .

Lemma 5.10 has a very intuitive diagrammatic interpretation. We explain it using the following example.

Example 5.11. Consider the partition $\mu=(5,5,4,2)$. Its Frobenius form is $(4,3,1$ | $3,2,0)$. We have $q_{1}=4, q_{2}=11$ and $q_{3}=15$. The diagram below should be interpreted in the following way: $A(\mu)(\operatorname{Bas}(j))$ is a linear combination of those vectors $\operatorname{Bas}\left(j^{\prime}\right)$ for which 
there is an arrow $\operatorname{Bas}(j) \rightarrow \operatorname{Bas}\left(j^{\prime}\right)$.

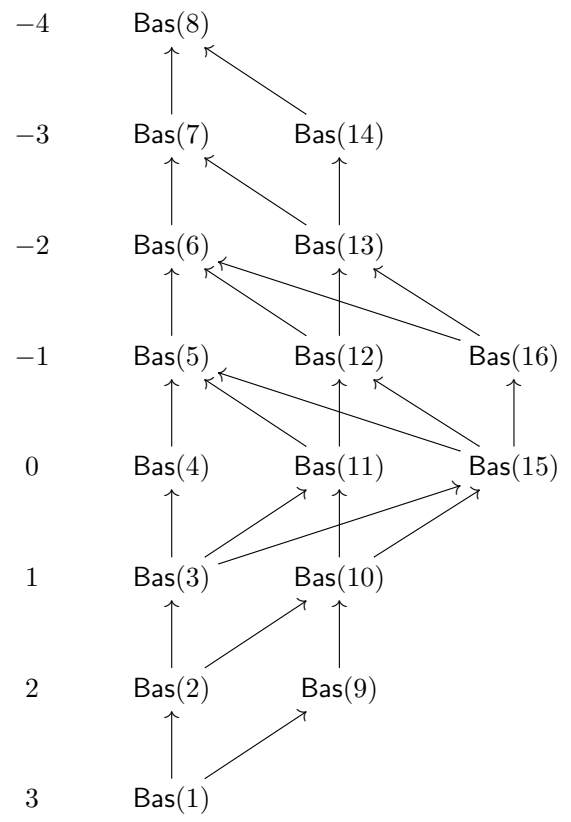

We have also introduced a numbering of the rows of the diagram. It is easy to see that $\operatorname{Bas}(j) \in \mathbf{V}_{i}^{\tau}$ if and only if $\operatorname{Bas}(j)$ lies in a row whose label is congruent to $i \bmod l$.

Lemma 5.12. Let $\mu \in \mathcal{P}_{\nu}(n l+|\nu|)$. Then $\mathbf{A}(\mu) \in \operatorname{Rep}\left(\overline{\mathbf{Q}}_{\infty}, \mathbf{d}\right)$.

Proof. We need to check that for each $0 \leq i \leq l-1$ the following holds:

$$
\operatorname{Im}\left(\left.A(\mu)\right|_{\mathbf{V}_{i}^{\tau}}\right) \subseteq \mathbf{V}_{i-1}^{\tau}, \quad \operatorname{Im}\left(\left.\Lambda(\mu)\right|_{\mathbf{V}_{i}^{\tau}}\right) \subseteq \mathbf{V}_{i+1}^{\tau}, \quad \operatorname{Im}(J(\mu)) \subseteq \mathbf{V}_{0}^{\tau}, \quad \bigoplus_{i=1}^{l-1} \mathbf{V}_{i}^{\tau} \subseteq \operatorname{ker}(I(\mu))
$$

Let us first show the first statement. We can draw a diagram as in Example 5.11. The subspace $\mathbf{V}_{i}^{\tau}$ has a basis consisting of vectors $\operatorname{Bas}(j)$ in rows labelled by numbers congruent to $i \bmod l$. The diagram shows that $A(\mu)(\operatorname{Bas}(j))$ is a linear combination of basis vectors in the row above $\operatorname{Bas}(j)$. But that row is labelled by a number congruent to $i-1 \bmod l$. Hence $A(\mu)(\operatorname{Bas}(j)) \in \mathbf{V}_{i-1}^{\tau}$. The argument for $\Lambda(\mu)$ is analogous.

Let us prove the last claim. Let $j \in\{1, \ldots, n l+|\nu|\}$ and suppose that $\operatorname{Bas}(j) \notin \mathbf{V}_{0}^{\tau}$. Let $1 \leq p \leq k$ and set $q_{p}=\sum_{s=1}^{p-1} m_{s}+r_{p}$. Since $\psi\left(q_{p}\right)=r_{p}-r_{p}=0$ we conclude that $p \notin\left\{q_{1}, \ldots, q_{k}\right\}$. But the only non-zero entries of $I(\mu)$ are those in columns numbered $q_{p}$, for $1 \leq p \leq k$. Hence $\operatorname{Bas}(j) \in \operatorname{ker} I(\mu)$. The calculation for $J(\mu)$ is similar.

Proposition 5.13. Let $\mu \in \mathcal{P}_{\nu}(n l+|\nu|)$. Then $\mathbf{A}(\mu) \in \mu_{\mathbf{d}}^{-1}(\theta)$.

Proof. By the previous lemma, we know that $\mathbf{A}(\mu) \in \operatorname{Rep}\left(\overline{\mathbf{Q}}_{\infty}, \mathbf{d}\right)$. Lemma 5.2 together with (19) implies that $[\Lambda(\mu), A(\mu)]+J(\mu) I(\mu)=\theta$, so $\mathbf{A}(\mu) \in \mu_{\mathbf{d}}^{-1}(\theta)$.

Theorem 5.14. Let $\mu \in \mathcal{P}_{\nu}(n l+|\nu|)$. Then $[\mathbf{A}(\mu)]:=G(\mathbf{d}) . \mathbf{A}(\mu)$ is a $\mathbb{C}^{*}$-fixed point in the quiver variety $\mathcal{X}_{\theta}(\mathbf{d})$.

Proof. Let $t \in \mathbb{C}^{*}$. We have $t . \mathbf{A}(\mu)=\left(t^{-1} \Lambda(\mu), t A(\mu), I(\mu), J(\mu)\right)$. We need to find a matrix $N$ in $G(\mathbf{d})$ such that $N t . \mathbf{A}(\mu) N^{-1}=\mathbf{A}(\mu)$.

For every $t \in \mathbb{C}^{*}$, let $Q(t)=\operatorname{diag}\left(1, t^{-1}, \ldots, t^{-n l-|\nu|+1}\right)$. Conjugating an $(n l+|\nu|) \times$ $(n l+|\nu|)$ matrix by $Q(t)$ multiplies the $j$-th diagonal by $t^{j}$. In particular, we have $Q(t)\left(\bigoplus_{i=1}^{k} t A\left(m_{i}, r_{i}\right)\right) Q(t)^{-1}=\bigoplus_{i=1}^{k} A\left(m_{i}, r_{i}\right)$ and $Q(t) t^{-1} \Lambda(\mu) Q(t)^{-1}=\Lambda(\mu)$.

Now consider the effect of conjugating $A(\mu)$ by $Q(t)$ on the off-diagonal block $A(\mu)^{i j}$ $(i \neq j)$. This block contains only one nonzero diagonal. Counting within the block, it is the diagonal labelled $r_{j}-r_{i}-1$. Counting inside the entire matrix $A(\mu)$, it is the diagonal labelled $q_{j}-q_{i}-1$. It follows that conjugation by $Q(t)$ multiplies the block $A(\mu)^{i j}$ by 
$t^{q_{j}-q_{i}-1}$. Hence we have

$$
Q(t)\left(\bigoplus_{1 \leq i \neq j \leq k} t A(\mu)^{i j}\right) Q(t)^{-1}=\bigoplus_{1 \leq i \neq j \leq k} t^{q_{j}-q_{i}} A(\mu)^{i j} .
$$

Let $P(t)=\bigoplus_{i=1}^{k} t^{q_{i}} \operatorname{Id}_{m_{i}}$. Conjugating $A(\mu)$ by $P(t)$ doesn't change the diagonal blocks but multiplies each off-diagonal block $A(\mu)^{i j}$ by $t^{q_{i}-q_{j}}$. We conclude that

$$
P(t) Q(t) t A(\mu) Q(t)^{-1} P(t)^{-1}=A(\mu) \text {. }
$$

Since the matrix $\Lambda(\mu)$ contains only diagonal blocks, conjugating by $P(t)$ doesn't have any impact. Hence

$$
P(t) Q(t) t^{-1} \Lambda(\mu) Q(t)^{-1} P(t)^{-1}=\Lambda(\mu) .
$$

The nonzero rows of $J(\mu)$ are precisely rows number $q_{1}, q_{2}, \ldots, q_{k}$. But the $q_{i}$-th entry of $P(t)$ is $t^{q_{i}}$ and the $q_{i}$-th entry of $Q(t)$ is $t^{1-q_{i}}$. Hence $P(t) Q(t) J(\mu)=t J(\mu)$. Similarly, $I(\mu) q(t)^{-1} P(t)^{-1}=t^{-1} I(\mu)$. Let $D(t)=t^{-1} \operatorname{Id}_{n l+|\nu|}$. Since $D(t)$ is a scalar matrix, conjugating by $D(t)$ doesn't change $A(\mu)$ or $\Lambda(\mu)$. On the other hand, $D(t) P(t) Q(t) J(\mu)=J(\mu)$ and $I(\mu) q(t)^{-1} P(t)^{-1} D(t)^{-1}=I(\mu)$.

The matrices $D(t), Q(t), P(t)$ are diagonal, so they represent linear automorphisms in $G(\mathbf{d})$. Hence $\mathbf{A}(\mu)$ and $t . \mathbf{A}(\mu)$ lie in the same $G(\mathbf{d})$-orbit, which is equivalent to saying that $\mathbf{A}(\mu)$ is a $\mathbb{C}^{*}$-fixed point in $\mathcal{X}_{\theta}(\mathbf{d})$.

5.4. Characters of the fibres of $\mathcal{V}$ at the fixed points. Recall the tautological bundle $\mathcal{V}_{\theta}(\mathbf{d})$ on $\mathcal{X}_{\theta}(\mathbf{d})$ from $\$ 3.2$. Let us abbreviate $\mathcal{V}:=\mathcal{V}_{\theta}(\mathbf{d})$. Let $\mathcal{V}_{\mu}$ denote the fibre of $\mathcal{V}$ at the fixed point $[\mathbf{A}(\mu)]:=G(\mathbf{d}) \cdot \mathbf{A}(\mu)$.

Proposition 5.15. Let $\mu \in \mathcal{P}_{\nu}(n l+|\nu|)$. Then

$$
\operatorname{ch}_{t} \mathcal{V}_{\mu}=\operatorname{Res}_{\mu}(t):=\sum_{\square \in \mu} t^{c(\square)} .
$$

Proof. Consider $[(\mathbf{A}(\mu), v)]:=G(\mathbf{d}) \cdot(\mathbf{A}(\mu), v) \in \mu_{\mathbf{d}}^{-1}(\theta) \times{ }^{G(\mathbf{d})} \widehat{\mathbf{V}}^{\tau}=\mathcal{V}$. We have

$$
\begin{aligned}
t .(\mathbf{A}(\mu), v)=(t . \mathbf{A}(\mu), v) & \sim\left(D(t) P(t) Q(t)(t . \mathbf{A}(\mu))(D(t) P(t) Q(t))^{-1},(D(t) P(t) Q(t))^{-1} v\right) \\
& =\left(\mathbf{A}(\mu), Q(t)^{-1} P(t)^{-1} D(t)^{-1} v\right) .
\end{aligned}
$$

The basis vectors $\{\operatorname{Bas}(1), \operatorname{Bas}(2), \ldots, \operatorname{Bas}(n l+|\nu|)\}$ are eigenvectors of $(D(t) P(t) Q(t))^{-1}$ with corresponding eigenvalues

$$
\left\{t^{1-r_{1}}, t^{2-r_{1}}, \ldots, t^{m_{1}-r_{1}} ; t^{1-r_{2}}, t^{2-r_{2}}, \ldots, t^{m_{2}-r_{2}} ; \ldots ; t^{1-r_{k}}, t^{2-r_{k}}, \ldots, t^{m_{k}-r_{k}}\right\} .
$$

Moreover, these eigenvalues are precisely the contents of the cells in the Young diagram of $\mu$, counting from the foot of the innermost Frobenius hook upward and later to the right, before passing to subsequent Frobenius hooks. Hence $\operatorname{ch}_{t} \mathcal{V}_{\mu}=\sum_{\square \in \mu} t^{c(\square)}$.

Recall that we have assumed that the parameter $\theta$ is chosen so that the variety $\mathcal{X}_{\theta}(\mathbf{d})$ is smooth. By [24, Proposition 22] there exists $w \in \tilde{S}_{l}$ such that $w * \mathbf{d}_{\tau}=0$. Let $w=\sigma_{i_{1}} \cdots \sigma_{i_{m}}$ be a reduced expression for $w$ in $\tilde{S}_{l}$. Furthermore, let $w \cdot \theta=\left(\vartheta_{0}, \ldots, \vartheta_{l-1}\right)$ and $H_{1}=\vartheta_{1}, \ldots, H_{l-1}=\vartheta_{l-1}, h=-\sum_{i=0}^{l-1} \vartheta_{i}, \mathbf{h}=\left(h, H_{1}, \ldots, H_{l-1}\right)$. Composing the Etingof-Ginzburg map with reflection functors we obtain a $\mathbb{C}^{*}$-equivariant isomorphism

Corollary 5.16. The map

$$
\mathcal{Y}_{\mathbf{h}} \stackrel{\mathrm{EG}}{\longrightarrow} \mathcal{C}_{\mathbf{h}}=\mathcal{X}_{w \cdot \theta}(n \delta) \stackrel{\mathfrak{R}_{i_{m}} \circ \cdots \circ \Re_{i_{1}}}{\longrightarrow} \mathcal{X}_{\theta}(\mathbf{d}) .
$$

$$
\mathcal{P}_{\nu}(n l+|\nu|) \rightarrow \mathcal{X}_{\theta}(\mathbf{d})^{\mathbb{C}^{*}}, \quad \mu \mapsto[\mathbf{A}(\mu)]:=G(\mathbf{d}) \cdot \mathbf{A}(\mu)
$$

is a bijection.

Proof. The $\mathbb{C}^{*}$-fixed points in $\operatorname{MaxSpec} Z_{\mathbf{h}}$ are in bijection with $l$-multipartitions of $n$, which are themselves in bijection with partitions of $n l+|\nu|$ with $l$-core $\nu$. But $\mathcal{Y}_{\mathbf{h}}$ is $\mathbb{C}^{*}$-equivariantly isomorphic to $\mathcal{X}_{\theta}(\mathbf{d})$, so $\left|\mathcal{X}_{\theta}(\mathbf{d})^{\mathbb{C}^{*}}\right|=\left|\left(\operatorname{MaxSpec} Z_{\mathbf{h}}\right)^{\mathbb{C}^{*}}\right|=|\mathcal{P}(l, n)|=$ $\left|\mathcal{P}_{\nu}(n l+|\nu|)\right|$.

Since a partition is uniquely determined by its residue, $\mu \neq \mu^{\prime} \operatorname{implies} \operatorname{ch}_{t} \mathcal{V}_{\mu} \neq \operatorname{ch}_{t} \mathcal{V}_{\mu^{\prime}}$, which in turn implies that $[\mathbf{A}(\mu)] \neq\left[\mathbf{A}\left(\mu^{\prime}\right)\right]$. It follows that 200 is a bijection because it is an injective function between sets of the same cardinality. 


\section{Degenerate affine Hecke Algebras}

In this section we use degenerate affine Hecke algebras and a version of the Chevalley restriction map to associate to each $\mathbb{C}^{*}$-fixed point in $\mathcal{Y}_{\mathbf{h}}$ and $\mathcal{C}_{\mathbf{h}}$ a distinct point in $\mathbb{C}^{n} / S_{n}$ in a manner which is compatible with the Etingof-Ginzburg isomorphism.

6.1. Degenerate affine Hecke algebras. Degenerate affine Hecke algebras associated to complex reflection groups of type $G(l, 1, n)$ were defined in 32 . Let us recall their definition and basic properties.

Definition 6.1. Let $\kappa \in \mathbb{C}$. The degenerate affine Hecke algebra associated to $\Gamma_{n}$ is the $\mathbb{C}$-algebra $\mathcal{H}_{\kappa}$ generated by $\Gamma_{n}$ and pairwise commuting elements $z_{1}, \ldots, z_{n}$ satisfying the following relations:

$$
\begin{gathered}
\epsilon_{j} z_{i}=z_{i} \epsilon_{j}(1 \leq i, j \leq n), \quad s_{i} z_{j}=z_{j} s_{i}(j \neq i, i+1), \\
s_{i} z_{i+1}=z_{i} s_{i}+\kappa \sum_{k=0}^{l-1} \epsilon_{i}^{-k} \epsilon_{i+1}^{k}(1 \leq i \leq n-1) .
\end{gathered}
$$

Let $\mathcal{Z}_{\kappa}$ denote the centre of $\mathcal{H}_{\kappa}$.

Proposition 6.2. The algebra $\mathcal{H}_{\kappa}$ has the following properties.

a) As a vector space, $\mathcal{H}_{\kappa}$ is canonically isomorphic to $\mathbb{C}\left[z_{1}, \ldots, z_{n}\right] \otimes \mathbb{C} \Gamma_{n}$.

b) There is an injective algebra homomorphism $\mathbb{C}\left[z_{1}, \ldots, z_{n}\right]^{S_{n}} \hookrightarrow \mathcal{Z}_{\kappa}$.

c) The algebra $\mathcal{H}_{\kappa}$ has a maximal commutative subalgebra $\mathfrak{C}_{\kappa}$ which is isomorphic to $\mathbb{C}\left[z_{1}, \ldots, z_{n}\right] \otimes \mathbb{C}(\mathbb{Z} / l \mathbb{Z})^{n}$

d) Suppose that $\mathbf{h}=\left(h, H_{1}, \ldots, H_{l-1}\right) \in \mathbb{Q}^{l}$ satisfies $h=\kappa$. Then there exists an injective algebra homomorphism $\mathcal{H}_{\kappa} \hookrightarrow \mathbb{H}_{\mathbf{h}}$ defined by

$$
g \mapsto g\left(g \in \Gamma_{n}\right), \quad z_{i} \mapsto y_{i} x_{i}+\kappa \sum_{1 \leq j<i} \sum_{k=0}^{l-1} s_{i, j} \epsilon_{i}^{k} \epsilon_{j}^{-k}+\sum_{k=1}^{l-1} c_{k} \sum_{m=0}^{l-1} \eta^{-m k} \epsilon_{i}^{m},
$$

where the $c_{k}$ 's are the parameters obtained from $\mathbf{h}$ as in [16, §2.7]. This homomorphism restricts to a homomorphism

$$
\mathbb{C}\left[z_{1}, \ldots, z_{n}\right]^{S_{n}} \hookrightarrow Z_{\mathbf{h}}
$$

Proof. See Propositions 1.1, 2.1, 2.3 and $§ 3.1$ in [9] as well as Proposition 10.1 and Corollary 10.1 in [18.

Let us recall the construction of some irreducible $\mathcal{H}_{\kappa}$-modules.

Definition 6.3. Let $a=\left(a_{1}, \ldots, a_{n}\right) \in \mathbb{C}^{n}$ and $b=\left(b_{1}, \ldots, b_{n}\right) \in(\mathbb{Z} / l \mathbb{Z})^{n}$. Let $\mathbb{C}_{a, b}$ be the one-dimensional representation of the commutative algebra $\mathfrak{C}_{\kappa}=\mathbb{C}\left[z_{1}, \ldots, z_{n}\right] \otimes$ $\mathbb{C}(\mathbb{Z} / l \mathbb{Z})^{n}$ defined by $z_{i} \cdot v=a_{i} v, \epsilon_{i} \cdot v=\eta^{b_{i}} v$ for each $1 \leq i \leq n$ and $v \in \mathbb{C}_{a, b}$. Define

$$
M(a, b):=\mathcal{H}_{\kappa} \otimes \mathfrak{c}_{\kappa} \mathbb{C}_{a, b} .
$$

Proposition 6.4 ([9, Theorem 4.9]). Let $a \in \mathbb{C}^{n}$ and $b \in(\mathbb{Z} / l \mathbb{Z})^{n}$. If $a_{i}-a_{j} \neq 0, \pm l \kappa$ for all $1 \leq i \neq j \leq n$ then the $\mathcal{H}_{\kappa}$-module $M(a, b)$ is irreducible.

6.2. Restricting $\mathbb{H}_{\mathbf{h}}$-modules to $\mathcal{H}_{h}$-modules. Fix $\mathbf{h} \in \mathbb{Q}^{l}$ such that $\mathcal{Y}_{\mathbf{h}}$ is smooth and set $\kappa=h$. We are going to consider the generic behaviour of simple modules over $\mathbb{H}_{\mathbf{h}}$ under the restriction functor to $\mathcal{H}_{h}$-modules.

Definition 6.5. Set

$$
\mathcal{D}:=\left\{a=\left(a_{1}, \ldots, a_{n}\right) \in \mathbb{C}^{n} \mid a_{i}-a_{j} \neq 0, \pm l \kappa \text { for all } 1 \leq i \neq j \leq n\right\} .
$$

Observe that $\mathcal{D}$ is a dense open subset of $\mathbb{C}^{n}$. Proposition 6.4 implies that for all $a \in \mathcal{D}$ and $b \in(\mathbb{Z} / l \mathbb{Z})^{n}$ the module $M(a, b)$ is irreducible. Consider the diagram

$$
\mathbb{C}^{n} \stackrel{\phi}{\longrightarrow} \mathbb{C}^{n} / S_{n} \stackrel{\rho_{1}}{\longleftarrow} \mathcal{Y}_{\mathbf{h}},
$$

where $\phi$ is the canonical map and $\rho_{1}$ is the dominant morphism induced by 22 . Set

$$
\mathcal{U}:=\rho_{1}^{-1}(\phi(\mathcal{D})) .
$$

Lemma 6.6. The subset $\mathcal{U}$ is open and dense in $\mathcal{Y}_{\mathbf{h}}$. 
Proof. The set $\mathcal{U}$ is open because $\phi$ is a quotient map and $\rho_{1}$ is continuous. Since the morphism $\rho_{1}$ is dominant, $\rho_{1}\left(\mathcal{Y}_{\mathbf{h}}\right)$ is dense in $\mathbb{C}^{n} / S_{n}$. Therefore, since $\phi(\mathcal{D})$ is open in $\mathbb{C}^{n} / S_{n}$, we have $\phi(\mathcal{D}) \cap \rho_{1}\left(\mathcal{Y}_{\mathbf{h}}\right) \neq \varnothing$. Hence $\mathcal{U}$ is nonempty. The fact that the variety $\mathcal{Y}_{\mathbf{h}}$ is irreducible (see e.g. [11, Corollary 3.9]) now implies that $\mathcal{U}$ is dense.

Let $\hat{e}=\frac{1}{(n-1) !} \sum_{g \in S_{n-1} \subset \Gamma_{n}} g$ and $\mathbf{0}=(0, \ldots, 0) \in(\mathbb{Z} / l \mathbb{Z})^{n}$. For the rest of this subsection fix an irreducible $\mathbb{H}_{\mathbf{h}}$-module $L$ whose support is contained in $\mathcal{U}$ (i.e. $\chi_{L} \in \mathcal{U}$ ). Consider $L$ as an $\mathcal{H}_{h}$-module using the embedding (21).

Lemma 6.7. There exists an injective homomorphism of $\mathcal{H}_{h}$-modules $M(a, \mathbf{0}) \hookrightarrow L$ for some $a \in \mathcal{D}$.

Proof. We have a $(\mathbb{Z} / l \mathbb{Z})^{n}$-module decomposition $L=\bigoplus_{b \in(\mathbb{Z} / l \mathbb{Z})^{n}} L(b)$, where $L(b)$ is the subspace of $L$ such that $\epsilon_{i} . w=\eta^{b_{i}} w$ for all $w \in L(b)$. Since the $z_{i}$ 's commute with the $\epsilon_{j}$ 's, each subspace $L(b)$ is preserved under the action of the $z_{i}$ 's. In particular, $z_{1}, \ldots, z_{n}$ define commuting linear operators on $L(\mathbf{0})$, so they have some common eigenvector $v \in L(\mathbf{0})$. Let $a_{1}, \ldots, a_{n}$ be the respective eigenvalues of the $z_{i}$ 's. Since the support of $L$ is contained in $\mathcal{U}$, we have $a=\left(a_{1}, \ldots, a_{n}\right) \in \mathcal{D}$.

Let $v_{a, \mathbf{0}} \in \mathbb{C}_{a, \mathbf{0}}$. Then the map $1 \otimes v_{a, \mathbf{0}} \mapsto v$ defines a non-zero $\mathcal{H}_{h}$-module homomorphism $M(a, \mathbf{0}) \rightarrow L$. Since $a=\left(a_{1}, \ldots, a_{n}\right) \in \mathcal{D}$, the module $M(a, \mathbf{0})$ is simple and so this homomorphism is injective.

Recall that $L^{\Gamma_{n-1}}$ is a module over $\left\langle\epsilon_{1}\right\rangle \cong \mathbb{Z} / l \mathbb{Z}$. Let $L_{\chi_{0}}^{\Gamma_{n-1}}$ denote the isotypic component corresponding to the trivial character.

Lemma 6.8. We have $\hat{e} M(a, \mathbf{0})=L_{\chi_{0}}^{\Gamma_{n-1}}$. Moreover, $\hat{e} M(a, \mathbf{0})$ is stable under the action of $z_{1}$ and the eigenvalues of $z_{1}$ on $\hat{e} M(a, \mathbf{0})$ are $a_{1}, \ldots, a_{n}$.

Proof. The action of $\epsilon_{1}$ on $M(a, \mathbf{0})$ is trivial by definition. We have a vector space isomorphism $M(a, \mathbf{0}) \cong \mathbb{C} S_{n} \otimes \mathbb{C}_{a, \mathbf{0}}$. Therefore $\left\{\hat{e} s_{1, j} \otimes v_{a, \mathbf{0}} \mid 1 \leq j \leq n\right\}$ form a basis of $\hat{e} M(a, \mathbf{0})$ for any nonzero $v_{a, \mathbf{0}} \in \mathbb{C}_{a, \mathbf{0}}$. In particular, $\operatorname{dim} \hat{e} M(a, \mathbf{0})=n$. Let us show that each of the basis elements we defined is fixed under the action of $\Gamma_{n-1}$. We first note that since for each $g \in S_{n-1} \subset \Gamma_{n}$ we have $g \hat{e}=\hat{e}$, the subgroup $S_{n-1}$ fixes each $\hat{e} s_{1, j} \otimes v_{a, \mathbf{0}}$. Now consider $\epsilon_{i} . \hat{e} s_{1, j} \otimes v_{a, \mathbf{0}}$ with $2 \leq i \leq n$. We have $\epsilon_{i} . \hat{e} s_{1, j} \otimes v_{a, \mathbf{0}}=\sum_{g \in S_{n-1}} g s_{1, j} \epsilon_{i(g)} \otimes v_{a, \mathbf{0}}$, where $i(g)$ is an index depending on $g$. But each $\epsilon_{i(g)}$ acts on $v_{a, 0}$ by the identity, so we conclude that $\epsilon_{i}$ fixes $\hat{e} s_{1, j} \otimes v_{a, \mathbf{0}}$. The stability of $\hat{e} M(a, \mathbf{0})$ under the action of $z_{1}$ follows from the fact that $z_{1}$ commutes with $\hat{e}$. The calculation of the eigenvalues is similar to the calculation in the proof of [2, Lemma 4.7].

6.3. Connection to the Etingof-Ginzburg isomorphism. Suppose that $L$ is an irreducible $\mathbb{H}_{\mathbf{h}}$-module whose support is contained in $\mathcal{U}$. Let $M(a, \mathbf{0})$ be as in Lemma 6.7 . Using Lemma 6.8 and (10) we can identify $\hat{e} M(a, \mathbf{0})=L_{\chi_{0}}^{\Gamma_{n-1}} \cong\left(\mathbb{C} \Gamma_{n}\right)^{\Gamma_{n-1}} \cong \mathbf{V}_{0}$. Suppose that $\mathrm{EG}\left(\chi_{L}\right)=[(\mathbf{X}, \mathbf{Y}, I, J)]$. The embedding $(21)$ sends

$$
z_{1} \quad \mapsto \quad x_{1} y_{1}+\sum_{k=1}^{l-1} c_{k} \sum_{m=0}^{l-1} \eta^{-m k} \epsilon_{1}^{m} .
$$

Since $\epsilon_{1}$ acts trivially on $M(a, \mathbf{0})$, the action of $z_{1}$ on $\hat{e} M(a, \mathbf{0})$ can be identified with the action of $y_{1} x_{1}$ on $L_{\chi_{0}}^{\Gamma_{n-1}}$. Using the Etingof-Ginzburg isomorphism, the latter can be identified, up to conjugation, with the matrix $Y_{1} X_{0}$.

Definition 6.9. Let $\rho_{2}: \mathcal{C}_{\mathbf{h}} \rightarrow \mathbb{C}^{n} / S_{n}$ be the morphism sending $[(\mathbf{X}, \mathbf{Y}, I, J)]$ to the multiset of the generalized eigenvalues of the matrix $Y_{1} X_{0}$.

Proposition 6.10. The following diagram commutes.

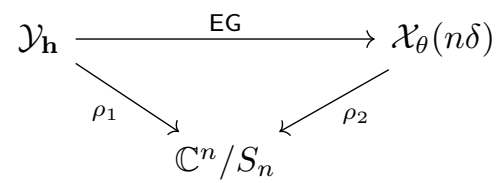

Proof. Since EG is an isomorphism, it suffices to show there exists a dense open subset of $\mathcal{Y}_{\mathbf{h}}$ for which the diagram commutes. Consider the dense open subset $\mathcal{U}$ from $(23)$. 
Since $\mathcal{Y}_{\mathbf{h}}$ is smooth, for each $\chi \in \mathcal{U}$, there exists a unique simple $\mathbb{H}_{\mathbf{h}}$-module $L$ such that $\chi=\chi_{L}$. Moreover, there is an injective $\mathcal{H}_{h}$-module homomorphism $M(a, \mathbf{0}) \hookrightarrow L$ for some $a \in \mathcal{D}$, by Lemma 6.7. Set $[(\mathbf{X}, \mathbf{Y}, I, J)]:=\mathrm{EG}\left(\chi_{L}\right)$. The remarks at the beginning of this subsection imply that the matrix $Y_{1} X_{0}$ describes the action of $z_{1}$ on $\hat{e} M(a, \mathbf{0})$. Hence the eigenvalues of $Y_{1} X_{0}$ are the same as the eigenvalues of the operator $\left.z_{1}\right|_{\hat{e} M(a, \mathbf{0})}$. By Lemma 6.8, these eigenvalues are $a_{1}, \ldots, a_{n}$. Hence $\rho_{2} \circ \operatorname{EG}\left(\chi_{L}\right)=\phi(a) \in \mathbb{C}^{n} / S_{n}$.

On the other hand, consider the composition

$$
\mathbb{C}\left[z_{1}, \ldots, z_{n}\right]^{S_{n}} \hookrightarrow Z_{\mathbf{h}} \stackrel{\chi_{L}}{\longrightarrow} \mathbb{C} .
$$

By the definition of $M(a, \mathbf{0})$, a symmetric polynomial $f\left(z_{1}, \ldots, z_{n}\right)$ acts on $1 \otimes \mathbb{C}_{a, \mathbf{0}}$ by the scalar $f\left(a_{1}, \ldots, a_{n}\right)$. Since $f\left(z_{1}, \ldots, z_{n}\right)$ is central in $\mathbb{H}_{\mathbf{h}}$, it acts by this scalar on all of $L$. Therefore, the kernel of (24) equals the maximal ideal in $\mathbb{C}\left[z_{1}, \ldots, z_{n}\right]^{S_{n}}$ consisting of those symmetric polynomials $f$ which satisfy $f\left(a_{1}, \ldots, a_{n}\right)=0$, which is the vanishing ideal of $\phi(a)$.

6.4. The images of the $\mathbb{C}^{*}$-fixed points in $\mathbb{C}^{n} / S_{n}$. We are now going to identify the images of the $\mathbb{C}^{*}$-fixed points under $\rho_{1}$ and $\rho_{2}$. Set $\mathbf{e}=\left(e_{0}, \ldots, e_{l-1}\right) \in \mathbb{Q}^{l}$, where $e_{0}=0$ and $e_{i}=\sum_{j=1}^{i} H_{j}$ for $i=1, \ldots, l-1$. Set $\theta:=\theta_{\mathbf{h}}$ as in $(9)$. For the rest of this section fix $\mu \in \mathcal{P}_{\varnothing}(n l)$. Let us identify a point $\left(a_{1}, \ldots, a_{n}\right) \in \mathbb{C}^{n} / S_{n}$ with the "polynomial" $\sum_{i=1}^{n} t^{a_{i}}$.

Lemma 6.11 ([28, §5.4]). Let $\underline{\lambda} \in \mathcal{P}(l, n)$. Then $\rho_{1}\left(\chi_{\underline{\lambda}}\right)=\operatorname{Res}_{\underline{\underline{\lambda}}}^{\mathbf{e}}\left(t^{h}\right)$.

Definition 6.12. Let $\mu=\left(a_{1}, \ldots, a_{k} \mid b_{1}, \ldots, b_{k}\right)$ be the Frobenius form of $\mu$. For each $1 \leq i \leq k$, let $r_{i}=b_{i}+1$ and $m_{i}=a_{i}+b_{i}+1$. Recall the matrices $\Lambda\left(m_{i}\right)$ and $A\left(m_{i}, r_{i}\right)$ from Definition 5.1. If $\Lambda\left(m_{i}\right) A\left(m_{i}, r_{i}\right)=\operatorname{diag}\left(\alpha_{1}, \alpha_{2}, \ldots, \alpha_{m_{i}-1}, \alpha_{m_{i}}\right)$, then we define

$$
\operatorname{Eig}(\mu, i)=\sum_{\substack{1 \leq j \leq m_{i}, j=r_{i}-1 \bmod l}} t^{\alpha_{j}}, \quad \operatorname{Eig}(\mu)=\sum_{i=1}^{k} \operatorname{Eig}(\mu, i) .
$$

Lemma 6.13. We have $\rho_{2}([\mathbf{A}(\mu)])=\operatorname{Eig}(\mu)$.

Proof. $\operatorname{Eig}(\mu)$ picks out exactly the eigenvalues of the restricted endomorphism $\left.\Lambda(\mu) A(\mu)\right|_{\mathbf{V}_{1}}$ from all the eigenvalues of $\Lambda(\mu) A(\mu)$. But these are the same as the eigenvalues of $\left.A(\mu) \Lambda(\mu)\right|_{\mathbf{v}_{0}}$. The fact that $\rho_{2}([\mathbf{A}(\mu)])=\operatorname{Eig}(\mu)$ now follows immediately from the definition of $\rho_{2}$.

By Proposition 6.10. Lemma 6.11. Lemma 6.13 and the fact that a multipartition is uniquely determined by its e-residue for generic $\mathbf{e}$, we have

$$
\operatorname{Eig}(\mu)=\rho_{2}([\mathbf{A}(\mu)])=\rho_{1}\left(\chi_{\underline{\lambda}}\right)=\operatorname{Res}_{\underline{\lambda}}^{\mathbf{e}}\left(t^{h}\right)
$$

for unique $\underline{\lambda} \in \mathcal{P}(l, n)$.

Definition 6.14. Define $\underline{\operatorname{Eig}}(\mu):=\left(\operatorname{Eig}(\mu)^{0}, \operatorname{Eig}(\mu)^{1}, \ldots, \operatorname{Eig}(\mu)^{l-1}\right) \in \mathcal{P}(l, n)$ by the equation

We thus have a bijection

$$
\operatorname{Res}_{\underline{\operatorname{Eig}(\mu)}}^{\mathbf{e}}\left(t^{h}\right)=\operatorname{Eig}(\mu)
$$

$$
\underline{\operatorname{Eig}}: \mathcal{P}_{\varnothing}(n l) \rightarrow \mathcal{P}(l, n), \quad \mu \mapsto \underline{\operatorname{Eig}}(\mu) .
$$

Proposition 6.15. Let $\mu \in \mathcal{P}_{\varnothing}(n l)$. The inverse of the Etingof-Ginzburg isomorphism sends the $\mathbb{C}^{*}$-fixed point $[\mathbf{A}(\mu)]$ in $\mathcal{C}_{\mathbf{h}}$ to the $\mathbb{C}^{*}$-fixed point $\chi_{\operatorname{Eig}(\mu)}$ in $\mathcal{Y}_{\mathbf{h}}$.

Proof. This follows directly from 25.

6.5. Calculation of $\operatorname{Eig}(\mu)$. Set $\mathbf{e}^{\prime}=\left(e_{0}^{\prime}, e_{1}^{\prime}, \ldots, e_{l-1}^{\prime}\right)$ and $\mathbf{e}^{\prime \prime}=\left(e_{0}^{\prime \prime}, e_{1}^{\prime \prime} \ldots, e_{l-1}^{\prime \prime}\right)$ with $e_{0}^{\prime}=-h, e_{l-1}^{\prime \prime}=0$ and

$$
e_{i}^{\prime}=e_{i} \quad(i=1, \ldots, l-1), \quad e_{i}^{\prime \prime}=h+\sum_{j=1}^{l-i-1} H_{j} \quad(i=0, \ldots, l-2) .
$$

In these notations all the lower indices are to be considered $\bmod l$. 
Lemma 6.16. Let $\mu=\left(a_{1}, \ldots, a_{k} \mid b_{1}, \ldots, b_{k}\right) \in \mathcal{P}_{\varnothing}(n l)$. Then

$$
\operatorname{Eig}(\mu)=\sum_{i=1}^{k}\left(\left(t^{e_{b_{i}}^{\prime}} \sum_{j=1}^{\left\lceil b_{i} / l\right\rceil} t^{-(j-1) h}\right)+\left(t^{e_{a_{i}}^{\prime \prime}} \sum_{j=1}^{\left\lfloor\left(a_{i}+1\right) / l\right\rfloor} t^{(j-1) h}\right)\right) .
$$

Proof. It suffices to show that for each $i=1, \ldots, k$ we have

$$
\operatorname{Eig}(\mu, i)=\left(t^{e_{b_{i}}^{\prime}} \sum_{j=1}^{\left\lceil b_{i} / l\right\rceil} t^{-(j-1) h}\right)+\left(t^{e_{a_{i}}^{\prime \prime}} \sum_{j=1}^{\left\lfloor\left(a_{i}+1\right) / l\right\rfloor} t^{(j-1) h}\right) .
$$

We can write

$$
\operatorname{Eig}(\mu, i):=\sum_{\substack{1 \leq j \leq m_{i}, j=r_{i}-1 \bmod l}} t^{\alpha_{j}}=\sum_{\substack{1 \leq j \leq r_{i}-1, j=r_{i}-1 \bmod l}} t^{\alpha_{j}}+\sum_{\substack{r_{i} \leq j \leq m_{i}, j=r_{i}-1 \bmod l}} t^{\alpha_{j}} .
$$

Note that $r_{i}-1=b_{i}=l \cdot\left(\left\lceil b_{i} / l\right\rceil-1\right)+d_{i}$, where $d_{i}$ is an integer such that $1 \leq$ $d_{i} \leq l$. The $j^{\prime} s$ satisfying $1 \leq j \leq r_{i}-1$ and $j=r_{i}-1 \bmod l$ are therefore precisely $b_{i}, b_{i}-l, b_{i}-2 l, \ldots, b_{i}-\left(\left\lceil b_{i} / l\right\rceil-1\right) \cdot l=d_{i}$. Recall that $\theta_{0}+\theta_{1}+\ldots+\theta_{l-1}=-h$. Hence $\alpha_{d_{i}+p l}=\alpha_{d_{i}}-p h$ for $p=0, \ldots,\left\lceil b_{i} / l\right\rceil-1$, by Definition 5.1. Therefore

$$
\sum_{\substack{1 \leq j \leq r_{i}-1, j=r_{i}=1 \bmod l}} t^{\alpha_{j}}=t^{\alpha_{d_{i}}} \sum_{j=1}^{\left\lceil b_{i} / l\right\rceil} t^{-(j-1) h}
$$

Observe that $d_{i}=b_{i} \bmod l$ if $1 \leq d_{i}<l$. Hence $\alpha_{d_{i}}=\sum_{j=1}^{d_{i}} \theta_{b_{i}+1-j}=\sum_{j=1}^{d_{i}} \theta_{d_{i}+1-j}=$ $\sum_{j=1}^{d_{i}} \theta_{j}=e_{d_{i}}^{\prime}=e_{b_{i}}^{\prime}$. If $d_{i}=l$ then $\alpha_{d_{i}}=\alpha_{l}=\sum_{j=1}^{l} \theta_{b_{i}+1-j}=\sum_{j=1}^{l} \theta_{j}=-h=e_{0}^{\prime}$. This shows that

$$
\sum_{\substack{1 \leq j \leq r_{i}-1, j=r_{i}=1 \bmod l}} t^{\alpha_{j}}=t^{e_{b_{i}}^{\prime}} \sum_{j=1}^{\left\lceil b_{i} / l\right\rceil} t^{-(j-1) h}
$$

Let us now consider the second sum on the RHS os [28). We have $m_{i}-r_{i}+1=a_{i}+1=$ $l \cdot\left\lfloor\left(a_{i}+1\right) / l\right\rfloor+c_{i}$ with $0 \leq c_{i}<l$. The $j^{\prime} s$ satisfying $r_{i} \leq j \leq m_{i}$ and $j=r_{i}-1 \bmod l$ are therefore precisely $b_{i}+l, b_{i}+2 l, \ldots, b_{i}+\left\lfloor\left(a_{i}+1\right) / l\right\rfloor \cdot l$. Note that $b_{i}+\left\lfloor\left(a_{i}+1\right) / l\right\rfloor \cdot l=m_{i}-c_{i}$. Hence $\alpha_{m_{i}-c_{i}-p l}=\alpha_{m_{i}-c_{i}}+p h$ for $p=0, \ldots,\left\lfloor\left(a_{i}+1\right) / l\right\rfloor-1$. One computes, in a similar fashion as above, that $\alpha_{m_{i}-c_{i}}=e_{a_{i}}^{\prime \prime}$. This shows that

$$
\sum_{\substack{r_{i} \leq j \leq m_{i}, j=r_{i}-1 \bmod l}} t^{\alpha_{j}}=t^{e_{a_{i}}^{\prime \prime}} \sum_{j=1}^{\left\lfloor\left(a_{i}+1\right) / l\right\rfloor} t^{(j-1) h} .
$$

\section{7. $\mathbb{C}^{*}$-FIXED POINTS UNDER THE ETINGOF-GINZBURG ISOMORPHISM}

We will now identify the multipartition $\operatorname{Eig}(\mu)$ and thereby establish the correspondence between the $\mathbb{C}^{*}$-fixed points under the Etingof-Ginzburg isomorphism.

7.1. The strategy. Our next goal is to show that $\operatorname{Eig}(\mu)=\underline{\operatorname{Quot}}(\mu)^{b}$. We will use the following strategy. Recall Lemma 4.7. We first prove that an analogous statement holds for the multipartition $\operatorname{Eig}(\mu)$. This will allow us to argue by induction on $n$. We then prove that $\underline{\operatorname{Eig}}(\mu)=\underline{Q u o t}(\mu)^{b}$ for partitions $\mu$ with the special property that only a unique $l$-rim-hook can be removed from $\mu$. We then deduce the result for arbitrary $\mu \in \mathcal{P}_{\varnothing}(n l)$.

7.2. Types and contributions of Frobenius hooks. We need to introduce some notation to break down formula (26) into simpler pieces. Throughout this section fix $\mu \in \mathcal{P}_{\varnothing}(n l)$.

Definition 7.1. Let $\mu=\left(a_{1}, \ldots, a_{k} \mid b_{1}, \ldots, b_{k}\right)$ be the Frobenius form of $\mu$. Let $F_{1}, \ldots, F_{k}$ be the Frobenius hooks in $\mathbb{Y}(\mu)$ so that $(i, i)$ is the root of $F_{i}$. Let

$$
\text { type }_{\mu}(L, i):=b_{i} \bmod l, \quad \operatorname{type}_{\mu}(A, i):=-\left(a_{i}+1\right) \bmod l \quad(i=1, \ldots, k) .
$$


We call the number type ${ }_{\mu}(L, i)$ the type of $\operatorname{leg}\left(F_{i}\right)$ and the number type ${ }_{\mu}(A, i)$ the type of $\operatorname{arm}\left(F_{i}\right)$. Let $\mathbf{e}, \mathbf{e}^{\prime}$ and $\mathbf{e}^{\prime \prime}$ be as in 6.4 and $\$ 6.5$. Define

$$
\Xi_{\mu}(L, i):=t^{e_{b_{i}}^{\prime}} \sum_{j=1}^{\left\lceil b_{i} / l\right\rceil} t^{-(j-1) h}, \quad \Xi_{\mu}(A, i):=t^{e_{a_{i}}^{\prime \prime}} \sum_{j=1}^{\left\lfloor\left(a_{i}+1\right) / l\right\rfloor} t^{(j-1) h} .
$$

We call $\Xi_{\mu}(L, i)$ the contribution of $\operatorname{leg}\left(F_{i}\right)$ and $\Xi_{\mu}(A, i)$ the contribution of $\operatorname{arm}\left(F_{i}\right)$.

By 27) we have

$$
\operatorname{Eig}(\mu, i)=\Xi_{\mu}(L, i)+\Xi_{\mu}(A, i)
$$

Lemma 7.2. We have

$$
t^{e_{j}} \operatorname{Res} \operatorname{Eig}(\mu)^{j}\left(t^{h}\right)=\sum_{\substack{1 \leq i \leq k, \operatorname{type}_{\mu}(A, i)=j}} \Xi_{\mu}(A, i)+\sum_{\substack{1 \leq i \leq k, \operatorname{type}_{\mu}(L, i)=j}} \Xi_{\mu}(L, i) .
$$

Proof. Each summand $t^{d}$ on the RHS of (26) corresponds (non-canonically) to a cell in the multipartition $\operatorname{Eig}(\mu)$ in the sense that it describes that cell's e-shifted content. For generic e we can write $t^{d}=t^{e_{i}} t^{c}$ for unique $i=0, \ldots, l-1$ and $c \in \mathbb{Z}$. The summand $t^{d}$ corresponds to a cell in the partition $\operatorname{Eig}(\mu)^{j}$ if and only if $i=j$, i.e., $t^{d}=t^{e_{j}} t^{c}$. Since $\operatorname{Eig}(\mu)=\sum_{p=1}^{k} \operatorname{Eig}(\mu, p)$, formula 29 implies that there exists an $1 \leq p \leq k$ such that $t^{d}$ is a summand in $\Xi_{\mu}(L, p)$ or $\Xi_{\mu}(A, p)$. In the former case $t^{d}=t^{e_{j}} t^{c}$ if and only if $j=b_{p} \bmod l=\operatorname{type}_{\mu}(L, p)$. In the latter case $t^{d}=t^{e_{j}} t^{c}$ if and only if $e_{a_{p}}^{\prime \prime}=h+e_{j}$, which is the case if and only if $j=-\left(a_{p}+1\right) \bmod l=\operatorname{type}_{\mu}(A, p)$.

7.3. Removal of rim-hooks. We will now investigate the effect of removing a rim-hook from $\mu$ on the multipartition $\underline{\operatorname{Eig}}(\mu)$. Let $\mathbb{Y}_{+/-/ 0}(\mu)$ denote the subset of $\mathbb{Y}(\mu)$ consisting of cells of positive/negative/zero content.

Lemma 7.3. Let $R$ be an l-rim-hook in $\mathbb{Y}(\mu)$ and suppose that $R \subset\left(\mathbb{Y}_{0}(\mu) \cup \mathbb{Y}_{+}(\mu)\right)$. Suppose that $R$ intersects $r$ Frobenius hooks, labelled $F_{p+1}, \ldots, F_{p+r}$ so that $(i, i)$ is the root of $F_{i}$. Let $\mu^{\prime}:=\mu-R$. Then

$$
\begin{gathered}
\text { type }_{\mu^{\prime}}(A, j)=\operatorname{type}_{\mu}(A, j+1), \quad \Xi_{\mu^{\prime}}(A, j)=\Xi_{\mu}(A, j+1) \quad(j=p+1, \ldots, p+r-1), \\
\operatorname{type}_{\mu^{\prime}}(A, p+r)=\operatorname{type}_{\mu}(A, p+1), \quad \Xi_{\mu^{\prime}}(A, p+r)=\Xi_{\mu}(A, p+1)-M,
\end{gathered}
$$

where $M$ is the (monic) monomial in $\Xi_{\mu}(A, p)$ of highest degree.

Proof. It is clear that $R$ must intersect adjacent Frobenius hooks. Recall that the residue of $R$ is of the form $\operatorname{Res}_{R}(t)=\sum_{i=i_{0}}^{i=i_{0}+l-1} t^{i}$ with $i_{0} \geq 0$. Moreover, we have

$$
\operatorname{Res}_{R \cap F_{j}}(t)=\sum_{i=i_{r+p-j}}^{i_{r+p-j+1}-1} t^{i}
$$

for some integers $i_{0}<i_{1}<\ldots<i_{r}=i_{0}+l$. One can easily see that these integers satisfy

$$
i_{r+p-j+1}-1=a_{j}
$$

where $a_{j}=\left|\operatorname{arm}\left(F_{j}\right)\right|=\max _{\square \in F_{j}} c(\square)$. Set $d_{j}:=\max _{\square \in F_{j}-R} c(\square)$. If $F_{j}-R=\varnothing$ set $d_{j}=-1$. From (31) and $(32)$ we easily deduce that

$$
d_{j}=a_{j+1} \quad(j=p+1, \ldots, p+r-1), \quad d_{p+r}=a_{p+1}-l .
$$

By definition, the type and contribution of $\operatorname{arm}\left(F_{j}\right)$ resp. $\operatorname{arm}\left(F_{j}-R\right)$ depend only on the numbers $a_{j}$ and $d_{j}$. The lemma now follows immediately from the definitions.

One can easily formulate a version of Lemma 7.3 for $R \subset\left(\mathbb{Y}_{0}(\mu) \cup \mathbb{Y}_{-}(\mu)\right)$. The proof is completely analogous. Lemma 7.3 admits the following graphical interpretation.

Example 7.4. The figure on the left shows the Young diagram of the partition $(5,5,4,3,3)$. The cells of content zero are marked as green. The blue cells form a 4 -rim-hook. The 
figure on the right shows the same Young diagram rearranged so that cells of the same content occupy the same row.
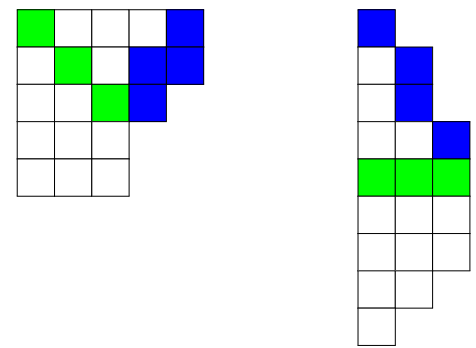

Using this visual representation we can easily determine the impact of removing the blue rim-hook. The length of the first arm after the removal equals the length of the second arm before the removal. Similarly, the length of the second arm after the removal equals the length of the third arm before the removal. Finally, the length of the third arm after the removal equals the length of the first arm before the removal minus four. This is precisely the content of Lemma 7.3 .

Proposition 7.5. Let $R$ be a rim-hook in $\mu$ and set $\mu^{\prime}:=\mu-R$. Then $\operatorname{Eig}\left(\mu^{\prime}\right)=\operatorname{Eig}(\mu)-$ for some $\boldsymbol{\square} \in \operatorname{Eig}(\mu)$.

Proof. There are three possibilities: $R \subset \mathbb{Y}_{0}(\mu) \cup \mathbb{Y}_{+}(\mu), R \subset \mathbb{Y}_{0}(\mu) \cup \mathbb{Y}_{-}(\mu)$ or $R \cap \mathbb{Y}_{+}(\mu) \neq$ $\varnothing, R \cap \mathbb{Y}_{-}(\mu) \neq \varnothing$. Consider the first case. Lemma 7.3 and Lemma 7.2 imply that there exists a $j \in\{0, \ldots, l-1\}$ such that $\operatorname{Eig}\left(\mu^{\prime}\right)^{i}=\operatorname{Eig}(\mu)^{2}$ if $i \neq j$ and $t^{e_{j}} \operatorname{Res} \operatorname{Eig}_{\left(\mu^{\prime}\right)^{j}}\left(t^{h}\right)=$ $t^{e_{j}} \operatorname{Res}_{\operatorname{Eig}(\mu)^{j}}\left(t^{h}\right)-t^{s_{j}} M$ for some monic monomial $M=t^{q h} \in \mathbb{Z}\left[t^{h}\right]$. Hence $\operatorname{Eig}\left(\mu^{\prime}\right)=$ $\operatorname{Eig}(\mu)-\square$ for some $\in \operatorname{Eig}(\mu)$ with $c(\mathbf{\square})=q$.

The second case is analogous. Now consider the third case. We claim that $\Xi_{\mu}(A, i)=0$ for every Frobenius hook $F_{i}$ whose arm intersects $R$ nontrivially. Indeed, by definition $\Xi_{\mu}(A, i) \neq 0$ only if $\left|\operatorname{arm}\left(F_{i}\right)\right|+1 \geq l$. We have $\left|\operatorname{arm}\left(F_{i}\right)\right|=\max _{\square \in \operatorname{arm}\left(F_{i}\right)} c(\square)=$ $\max _{\square \in \operatorname{arm}\left(F_{i}\right) \cap R} c(\square)$. Hence $\left|\operatorname{arm}\left(F_{i}\right)\right| \leq \max _{\square \in R} c(\square)$. However, since $R \cap \mathbb{Y}_{-}(\mu) \neq \varnothing$, the rim-hook $R$ must contain a cell of content -1 . The fact that $\operatorname{Res}_{R}(t)=t^{q} \sum_{p=0}^{l-1} t^{p}$ for some $q \in \mathbb{Z}$ implies that $\max _{\square \in R} c(\square) \leq l-2$. Hence $\left|\operatorname{arm}\left(F_{i}\right)\right|+1 \leq l-1$ and so $\Xi(A, i)=0$. Therefore the removal of $R$ does not affect the contribution of the arm of any Frobenius hook.

Now set $R^{\prime}:=R \cap\left(\mathbb{Y}_{0}(\mu) \cup \mathbb{Y}_{-}(\mu)\right)$. We have reduced the third case back to the second case, with the modification that $R^{\prime}$ is now a truncated rim-hook. We can still apply Lemma 7.3 with minor adjustments. In particular, equations $(33)$ are still true with the exception that the final equation becomes $d_{p+r}=0$. Let $j$ be the smallest integer such that $\operatorname{leg}\left(F_{j}\right) \cap R \neq \varnothing$. Using the same argument as before, we conclude that $t^{e_{j}} \operatorname{Res}_{\operatorname{Eig}\left(\mu^{\prime}\right)^{j}}\left(t^{h}\right)=t^{e_{j}} \operatorname{Res}_{\operatorname{Eig}(\mu)^{j}}\left(t^{h}\right)-t^{e_{j}-h}$ and $\operatorname{Eig}\left(\mu^{\prime}\right)^{i}=\operatorname{Eig}(\mu)^{i}$ if $i \neq j$.

7.4. Partitions with a unique removable rim-hook. In this section we show that $\operatorname{Eig}(\mu)=\underline{Q u o t}(\mu)^{b}$ for a certain class of partitions which we call $l$-special.

Definition 7.6. We say that a partition $\mu$ is $l$-special if the rim of $\mathbb{Y}(\mu)$ contains a unique $l$-rim-hook $R$. We call $R$ the unique removable l-rim-hook in $\mathbb{Y}(\mu)$. Let $\mathcal{P}_{\varnothing}^{s p}(k)$ denote the set of partitions of $k$ which are $l$-special and have a trivial $l$-core.

Our goal now is to describe partitions of $n l$ which are $l$-special and have a trivial $l$-core. Throughout this subsection we assume that $\mu \in \mathcal{P}_{\varnothing}^{s p}(n l)$. We let $R$ denote the unique removable $l$-rim-hook in $\mathbb{Y}(\mu)$ and set $\mu^{\prime}:=\mu-R$. Sometimes, for the sake of brevity, we will just write "rim-hook" instead of $l$-rim-hook.

Lemma 7.7. Let $\mu \in \mathcal{P}_{\varnothing}^{s p}(n l)$. Then:

a) Every column of $\mathbb{B}(\mu)$ contains the same number of beads.

b) Sliding distinct beads up results in the removal of distinct l-rim-hooks from $\mathbb{Y}(\mu)$.

c) The bead diagram $\mathbb{B}(\mu)$ contains $l-1$ columns with no gaps and one column with a unique string of adjacent gaps.

Proof. (a) The empty bead diagram describes the trivial partition. But bead diagrams which describe the trivial partition and have the property that the number of beads in 
the diagram is divisible by $l$ are unique up to adding or deleting full rows at the top of the diagram. Hence any such diagram consists of consecutive full rows at the top. Since $\mu$ has a trivial $l$-core, the process of sliding beads upward in $\mathbb{B}(\mu)$ must result in a bead diagram of this shape. But this is only possible if every column of $\mathbb{B}(\mu)$ contains the same number of beads.

(b) We can see this by considering the quotients of partitions corresponding to the bead diagrams obtained by moving up distinct beads. If the beads moved are on distinct runners, then a box is removed from distinct partitions in Quot $(\mu)$, so distinct multipartitions arise. If the beads are on the same runner, sliding upward distinct beads implies changing the first-column hook lengths in different ways in the same partition, so different multipartitions arise as well. But a trivial-core partition is uniquely determined by its quotient, so these distinct multipartitions are quotients of distinct partitions of $l(n-1)$.

(c) Since only one rim-hook can be removed from $\mu$, only one bead in our bead diagram can be moved upward. This implies that $l-1$ runners contain no gaps (i.e. they contain a consecutive string of beads counting from the top). The remaining runner must contain a unique gap or a unique string of gaps.

Lemma 7.8. The bead diagram $\mathbb{B}(\mu)$ can be decomposed into three blocks $A, B$ and $C$, counting from the top. Each block consists of identical rows. Rows in block A are full except for one bead. Let's say that the gap due to the absent bead is on runner $k$. Rows in block $B$ are either all full or all empty. Rows in block $C$ are empty except for one bead on runner $k$. Moreover, the number of rows in block $A$ equals the number of rows in block $C$.

Proof. This is an immediate consequence of Lemma 7.7.

Example 7.9. Let $l=3$. According to Lemma 7.8 the following bead diagrams correspond to 3 -special partitions:
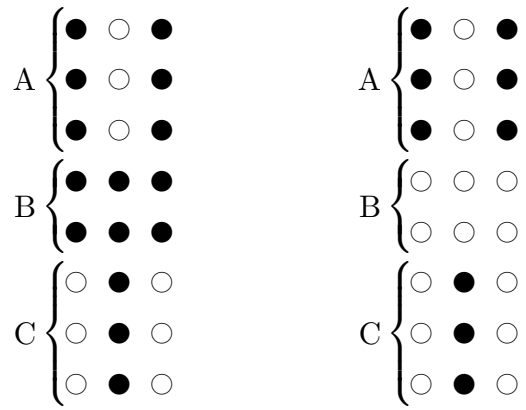

The bead diagram on the left describes the partition $\left(8,6,4,3^{7}, 2^{2}, 1^{2}\right)$ while the bead diagram on the right describes the partition $\left(14,12,10,3,2^{2}, 1^{2}\right)$.

In the sequel we will only consider the case where all the rows in block $B$ are full. All the following claims can easily be adapted to the case of empty rows.

Lemma 7.10. Suppose that block $A$ of $\mathbb{B}(\mu)$ has $m$ rows and block $B$ of $\mathbb{B}(\mu)$ has $p$ rows. Then $\mathbb{Y}(\mu)$ can be decomposed into four blocks $\hat{A}, \hat{B}, \hat{C}, \hat{D}$ :

- $\hat{A}$ is the Young diagram of the partition corresponding to the bead diagram $A$.

- $\hat{B}$ is a rectangle consisting of $m$ columns and $l \cdot p$ rows.

- If $k \neq 0$ block $\hat{C}$ is the Young diagram of the partition corresponding to the bead diagram $C$. If $k=0$ block $\hat{C}$ is the the Young diagram of the partition corresponding to the bead diagram obtained from $C$ by inserting an extra row at the top, which is full except for the empty point in column $l-1$.

- Block $\hat{D}$ is a square with $m$ rows and columns.

We recover $\mathbb{Y}(\mu)$ from these blocks by placing $\hat{A}$ at the bottom, stacking $\hat{B}$ on top, then stacking $\hat{D}$ on top and finally placing $\hat{C}$ on the right hand side of $\hat{D}$.

Proof. This follows from Lemma 7.8 by a routine calculation - one merely has to recover the first column hook lengths from the positions of the beads. 
Example 7.11. Consider the first bead diagram from Example 7.9. Below we illustrate the block decomposition of the corresponding Young diagram as in Lemma 7.10.

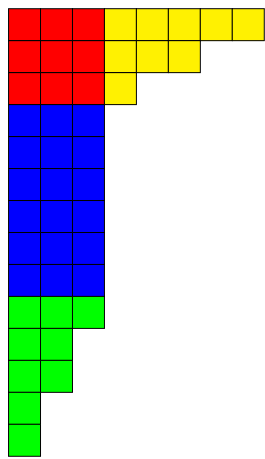
Block $\hat{A}$
Block $\hat{B}$
Block $\hat{C}$
Block $\hat{D}$

We are now ready to investigate the effect of removing the rim-hook $R$.

Lemma 7.12. The partition $\mu$ can be decomposed into $m$ Frobenius hooks. The unique removable rim-hook lies on the outermost Frobenius hook.

Proof. By Lemma 7.10 the 0 -th diagonal of the Young diagram of $\mu$ is contained in $\hat{D}$ and contains $m$ boxes. Hence there are $m$ Frobenius hooks. It follows easily from Lemma 7.8 and Lemma 7.10 that the outermost Frobenius hook $F_{m}$ in $\mu$ is the partition of the form $\left(k+1,1^{l \cdot p+l-k-1}\right)$. In particular, it contains $l \cdot(p+1) \geq l$ cells. Let us first consider $F_{m}$ as a partition in its own right and check whether it contains an $l$-rim-hook. If $p=0$ then $F_{m}$ is itself a rim-hook. If $p>1$ then the subset $1^{l \cdot p+l-k-1}$ of $F_{m}$ contains a rim-hook. Hence, in either case, $F_{m}$ contains a rim-hook. But a rim-hook of the outermost Frobenius hook $F_{m}$ is also a rim-hook of $\mu$ (because the outermost Frobenius hook is part of the rim).

Proposition 7.13. We have $\operatorname{Eig}\left(\mu^{\prime}\right)=\operatorname{Eig}(\mu)-\boldsymbol{\square}$, where $\boldsymbol{\square}$ is the unique removable cell in $\operatorname{Eig}(\mu)^{l-k-1}$ with content $-\overline{p \text {. }}$

Proof. The outermost Frobenius hook $F_{m}$ in $\mu$ is the partition of the form $\left(k+1,1^{l \cdot p+l-k-1}\right)$. By removing the rim-hook $R$ we obtain the partition $\left(k+1,1^{l \cdot p-k-1}\right)$ if $p \geq 1$ or the trivial partition if $p=0$. Since the rim-hook $R$ is contained in the outermost Frobenius hook, its removal does not affect the type and contribution of the arms and legs of the other Frobenius hooks.

There are several cases to be considered. Let $a_{m}=\left|\operatorname{arm}\left(F_{m}\right)\right|$ and $b_{m}=\left|\operatorname{leg}\left(F_{m}\right)\right|$. If $k \neq l-1$, then $\Xi_{\mu}(L, m)=t^{e_{b_{m} \bmod l}^{\prime}} \sum_{i=0}^{p} t^{-i h}$ and type ${ }_{\mu}(L, m)=b_{m}=l-k-1$ (while $\left.\Xi_{\mu}(A, m)=0\right)$. If $k=l-1$ and $p>0$ then $\Xi_{\mu}(L, m)=\sum_{i=1}^{p} t^{-i h}$ and type ${ }_{\mu}(L, m)=0$ (while $\Xi_{\mu}(A, m)=1$ ). If $k=l-1, p=0$ then $\Xi_{\mu}(A, m)=1$ and type ${ }_{\mu}(A, m)=0$ (while $\left.\Xi_{\mu}(L, m)=0\right)$.

Upon removing the rim-hook the polynomials listed above change as follows. We have $\Xi_{\mu^{\prime}}(L, m)=t^{e_{b_{m} \bmod l}^{\prime}} \sum_{i=0}^{p-1} t^{-i h}$ in the first case, $\Xi_{\mu^{\prime}}(L, m)=\sum_{i=1}^{p-1} t^{-i h}$ in the second case and $\Xi_{\mu^{\prime}}(A, m)=0$ in third case. The types do not change. We observe that in each case a monomial of degree $t^{-p h}$ (up to a shift) is subtracted, which corresponds to removing a cell of content $-p$ in $\operatorname{Eig}(\mu)^{l-k-1}$.

We obtain an analogous result for the multipartition Quot $(\mu)$.

Lemma 7.14. The following hold:

a) $\operatorname{Quot}(\mu)=\left(Q^{0}(\mu), \ldots, Q^{l-1}(\mu)\right)$ is a multipartition consisting of $l-1$ trivial partitions and one non-trivial partition.

b) Suppose that the $k$-th column in $\mathbb{B}(\mu)$ is the unique column which contains gaps. Then $Q^{k}(\mu)$ is the unique non-trivial partition in $\mathrm{Quot}(\mu)$. If that column has a string of $m$ gaps followed by a string of $q=m+p$ beads then the Young diagram of $Q^{k}(\mu)$ is a rectangle consisting of $m$ columns and $q$ rows.

Proof. The $l$-quotient of $\mu$ can be deduced directly from the bead diagram $\mathbb{B}(\mu)$. The description of the latter in Lemma 7.8 immediately implies the present lemma. 
Recall that $\mu^{\prime}:=\mu-R$, where $R$ is the unique rim-hook which can be removed from $\mu$.

Lemma 7.15. We have Quot $\left(\mu^{\prime}\right)=\underline{\text { Quot }}(\mu)-\square$, where $\square$ is the box in the bottom right corner of the rectangle described in Lemma 7.14. That box has content $-p$.

Proof. This is the only cell which can be removed from Quot $(\mu)$ by Lemma 7.14, so the claim now follows from Lemma 4.7.

The lemma implies in particular that Quot $\left(\mu^{\prime}\right)^{b}=\underline{\text { Quot }}(\mu)^{b}-\square$, where $\square$ is a box of content $-p$ in the $(l-k-1)$-th partition in $\underline{\text { uot }}(\mu)^{b}$.

7.5. Induction. We will now use induction on $n$ to show that $\underline{\operatorname{Quot}}(\mu)^{b}=\underline{\operatorname{Eig}}(\mu)$. There are two cases to be considered: $\mu \in \mathcal{P}_{\varnothing}^{s p}(n l)$ and $\mu \notin \mathcal{P}_{\varnothing}^{s p}(n l)$.

Proposition 7.16. Suppose that $\underline{\mathrm{Quot}}(\lambda)^{b}=\underline{\operatorname{Eig}}(\lambda)$ for all partitions $\lambda \vdash l(n-1)$ with trivial l-core. Let $\mu \in \mathcal{P}_{\varnothing}^{s p}(n l)$. Then $\underline{\operatorname{Quot}}(\mu)^{b} \overline{=} \underline{\operatorname{Eig}}(\mu)$.

Proof. By induction, Quot $\left(\mu^{\prime}\right)^{b}=\underline{\operatorname{Eig}}\left(\mu^{\prime}\right)$. But by Proposition 7.13 and Lemma 7.15, both $\underline{\text { Quot }}(\mu)^{b}$ and $\underline{\operatorname{Eig}}(\mu)$ arise from $\underline{\mathrm{Quot}}\left(\mu^{\prime}\right)^{\mathrm{b}}=\underline{\operatorname{Eig}}\left(\mu^{\prime}\right)$ by adding a box of content $-p$ to the $\overline{(l-k}-1)$-th partition. Hence $\underline{\underline{Q u o t}}(\mu)^{b}=\overline{\operatorname{Eig}}(\mu)$.

Proposition 7.17. Suppose that $\underline{\mathrm{Quot}}(\lambda)^{b}=\underline{\operatorname{Eig}}(\lambda)$ for all partitions $\lambda \vdash l(n-1)$ with trivial l-core. Let $\mu \notin \mathcal{P}_{\varnothing}^{s p}(n l)$. Then $\underline{\operatorname{Quot}}(\mu)^{b}=\underline{\operatorname{Eig}}(\mu)$.

Proof. Since $\mu \notin \mathcal{P}_{\varnothing}^{s p}(n l)$ we can remove two distinct (but possibly overlapping) rimhooks $R^{\prime}$ and $R^{\prime \prime}$ from $\mu$. Let $\mu^{\prime}=\mu-R^{\prime}$ and $\mu^{\prime \prime}=\mu-R^{\prime \prime}$. Then $\mu^{\prime} \neq \mu^{\prime \prime}$ and so Quot $\left(\mu^{\prime}\right) \neq \operatorname{Quot}\left(\mu^{\prime \prime}\right)$ (because the quotient of a partition with trivial core determines that partition uniquely). By Lemma 4.7, we have

$$
\underline{\operatorname{Quot}}\left(\mu^{\prime}\right)^{b}=\underline{\operatorname{Quot}}(\mu)^{b}-\square, \quad \underline{\operatorname{Quot}}\left(\mu^{\prime \prime}\right)^{b}=\underline{\operatorname{Quot}}(\mu)^{b}-\hat{\square}
$$

with $\square \neq \hat{\square} \in \underline{\text { Quot }}(\mu)^{b}$. By Proposition 7.5. we have

$$
\underline{\operatorname{Eig}}\left(\mu^{\prime}\right)=\underline{\operatorname{Eig}}(\mu)-\mathbf{\square}, \quad \underline{\operatorname{Eig}}\left(\mu^{\prime \prime}\right)=\underline{\operatorname{Eig}}(\mu)-
$$

for some $\boldsymbol{\square}, \hat{\boldsymbol{a}} \in \operatorname{Eig}(\mu)$. We know that Eig establishes a bijection between $l$-partitions of $n-1$ and partitions of $l(n-1)$ with a trivial $l$-core. Hence $\mathbf{\square} \neq \hat{\mathbf{a}}$. By the inductive hypothesis in our lemma,

$$
\underline{\operatorname{Quot}}\left(\mu^{\prime}\right)^{b}=\underline{\operatorname{Eig}}\left(\mu^{\prime}\right), \quad \underline{\operatorname{Quot}}\left(\mu^{\prime \prime}\right)^{b}=\underline{\operatorname{Eig}}\left(\mu^{\prime \prime}\right) .
$$

Hence

and so

$$
\underline{\operatorname{Quot}}(\mu)^{b}-\square=\underline{\operatorname{Eig}}(\mu)-\mathbf{\square}, \quad \underline{\operatorname{Quot}}(\mu)^{b}-\hat{\square}=\underline{\operatorname{Eig}}(\mu)-
$$

$$
\underline{\operatorname{Eig}}(\mu)=\underline{\operatorname{Quot}}(\mu)^{b}-\square+\mathbf{\square}=\underline{\operatorname{Quot}}(\mu)^{b}-\hat{\square}+\hat{\mathbf{n}}
$$

Since $\square \neq \hat{\square}$ and $\boldsymbol{\square} \neq \hat{\mathbf{Q}}$ we conclude that $\square=\boldsymbol{\square}$ and $\hat{\square}=\hat{\mathbf{\theta}}$. Therefore $\underline{\operatorname{Eig}}(\mu)=$ $\underline{\mathrm{Quot}}(\mu)^{b}$.

Theorem 7.18. Let $\mu \in \mathcal{P}_{\varnothing}(n l)$. Then $\underline{Q u o t}(\mu)^{b}=\operatorname{Eig}(\mu)$. The bijection between the labelling sets of $\mathbb{C}^{*}$-fixed points induced by the Etingof-Ginzburg isomorphism is given by

$$
\mathcal{P}(l, n) \rightarrow \mathcal{P}_{\varnothing}(n l), \quad \underline{\text { Quot }}(\mu)^{b} \mapsto \mu .
$$

Proof. The first claim follows directly from Propositions 7.16 and 7.17. The second claim follows from Proposition 6.15.

Remark 7.19. As a corollary, we also obtain the following explicit formula for the residue of the $l$-quotient of a partition $\mu=\left(a_{1}, \ldots, a_{k} \mid b_{1}, \ldots, b_{k}\right) \in \mathcal{P}_{\varnothing}(n l)$. Let us write $\underline{\text { Quot }}(\mu)=\left(Q^{0}, \ldots, Q^{l-1}\right)$. Then

$$
\operatorname{Res}_{Q^{l-j-1}}(t)=\sum_{\substack{1 \leq i \leq k,-\left(a_{i}+1\right)=j \bmod l}} t^{p_{a_{i}}} \sum_{m=1}^{\left\lfloor\left(a_{i}+1\right) / l\right\rfloor} t^{(m-1)}+\sum_{\substack{1 \leq i \leq k, b_{i}=j \bmod l}} t^{p_{b_{i}}^{\prime}} \sum_{m=1}^{\left\lceil b_{i} / l\right\rceil} t^{-(m-1)},
$$


where $p_{i}=1$ for $i=0, \ldots, l-2$ and $p_{l-1}=0$ while $p_{0}^{\prime}=-1$ and $p_{i}^{\prime}=0$ for $i=1, \ldots, l-1$. Indeed, the RHS of the formula above equals $\operatorname{Res}_{\operatorname{Eig}(\mu)^{j}}(t)$ by $(30)$. But $\operatorname{Eig}(\mu)^{j}=Q^{l-j-1}$ by Theorem 7.18 .

\section{The Higher LEVEL $q$-HOOK FORMUlA}

We will now use Theorem 7.18 to obtain the following "higher-level" generalization of the $q$-hook formula.

Theorem 8.1. Let $\mu \in \mathcal{P}_{\varnothing}(n l)$. Then

$$
\sum_{\square \in \mu} t^{c(\square)}=[n l]_{t} \sum_{\underline{\lambda} \uparrow \underline{\operatorname{Quot}}(\mu)^{b}} \frac{f_{\underline{\lambda}}(t)}{f_{\text {Quot }}(\mu)^{b}(t)} .
$$

Our proof is based on comparing the $\mathbb{C}^{*}$-characters of the vector bundles $\mathcal{V}_{\mathbf{h}}$ and $\mathcal{R}_{\mathbf{h}}^{\Gamma_{n-1}}$, and uses Proposition 5.15. Theorem 7.18 as well as the Etingof-Ginzburg isomorphism. The remaining ingredient is a calculation of the $\mathbb{C}^{*}$-characters of the fibres $\left(\mathcal{R}_{\mathbf{h}}^{\Gamma_{n-1}}\right)_{\underline{\lambda}}$ (see $\$ 2.4$. We carry out this calculation below. We first identify $\left(\mathcal{R}_{\mathbf{h}}^{\Gamma_{n-1}}\right)_{\underline{\lambda}}$ with a graded shift of $e_{n-1} L(\underline{\lambda})$. We next recall the graded multiplicity with which $L(\underline{\lambda})$ occurs in $\Delta(\underline{\lambda})$ and calculate the character of $e_{n-1} \Delta(\underline{\lambda})$. Finally, we use the equation

$$
\operatorname{ch}_{t} e_{n-1} L(\underline{\lambda})=\frac{\operatorname{ch}_{t} e_{n-1} \Delta(\underline{\lambda})}{[\Delta(\underline{\lambda}): L(\underline{\lambda})]_{\mathrm{gr}}} .
$$

Note that, setting $l=1$ in (34), we recover the usual $q$-hook formula, so our result also gives a new geometric proof of this well-known combinatorial identity.

8.1. Coinvariant algebras and fake degree polynomials. The algebra $\mathbb{C}[\mathfrak{h}]^{c o \Gamma_{n}}$ is a graded $\Gamma_{n}$-module. It is well known that $\mathbb{C}[\mathfrak{h}]^{c o \Gamma_{n}}$ is isomorphic to the regular representation $\mathbb{C} \Gamma_{n}$ as an ungraded $\Gamma_{n}$-module.

Let $\left.\mathbb{C}[\mathfrak{h}]^{c o \Gamma_{n}}\right|_{\Gamma_{n-1}}$ denote the restriction of $\mathbb{C}[\mathfrak{h}]^{c o \Gamma_{n}}$ to a $\Gamma_{n-1}$-module. Let $\mathfrak{h}^{\prime} \subset \mathfrak{h}$ denote the subspace spanned by $y_{2}, \ldots, y_{n}$. We choose a splitting $\mathfrak{h} \rightarrow \mathfrak{h}^{\prime}$ with kernel spanned by $y_{1}$. This splitting induces an inclusion $\mathbb{C}\left[\mathfrak{h}^{\prime}\right] \subset \mathbb{C}[\mathfrak{h}]$.

Lemma 8.2. We have an isomorphism of graded $\Gamma_{n-1}$-modules

$$
\left.\mathbb{C}[\mathfrak{h}]^{c o \Gamma_{n}}\right|_{\Gamma_{n-1}} \cong \mathbb{C}\left[\mathfrak{h}^{\prime}\right]^{c o \Gamma_{n-1}} \otimes U,
$$

where $U$ is a graded vector space with Poincaré polynomial $\mathrm{ch}_{t} U=[n]_{t}$.

Proof. We have a sequence of inclusions of graded $\Gamma_{n-1}$-modules

$$
\mathbb{C}[\mathfrak{h}]^{\Gamma_{n}} \hookrightarrow \mathbb{C}[\mathfrak{h}]^{\Gamma_{n-1}} \hookrightarrow \mathbb{C}[\mathfrak{h}]
$$

such that each ring is a free graded module over the previous ring. Hence there is an isomorphism of graded $\Gamma_{n-1}$-modules

$$
\mathbb{C}[\mathfrak{h}] /\left\langle\mathbb{C}[\mathfrak{h}]_{+}^{\Gamma_{n}}\right\rangle \cong \mathbb{C}[\mathfrak{h}] /\left\langle\mathbb{C}[\mathfrak{h}]_{+}^{\Gamma_{n-1}}\right\rangle \otimes \mathbb{C}[\mathfrak{h}]^{\Gamma_{n-1}} /\left\langle\mathbb{C}[\mathfrak{h}]_{+}^{\Gamma_{n}}\right\rangle .
$$

Observe that there is also an isomorphism of graded $\Gamma_{n-1}$-modules

$$
\mathbb{C}[\mathfrak{h}] /\left\langle\mathbb{C}[\mathfrak{h}]_{+}^{\Gamma_{n-1}}\right\rangle \cong \mathbb{C}\left[\mathfrak{h}^{\prime}\right] /\left\langle\mathbb{C}\left[\mathfrak{h}^{\prime}\right]_{+}^{\Gamma_{n-1}}\right\rangle=\mathbb{C}\left[\mathfrak{h}^{\prime}\right]^{c o \Gamma_{n-1}} .
$$

To prove the lemma it now suffices to find the Poincaré polynomial of the graded vector space $\mathbb{C}[\mathfrak{h}]^{\Gamma_{n-1}} /\left\langle\mathbb{C}[\mathfrak{h}]_{+}^{\Gamma_{n}}\right\rangle$. We know that $\mathbb{C}[\mathfrak{h}]^{\Gamma_{n-1}}$ is a polynomial algebra with generators in degrees $l, 2 l, \ldots,(n-1) l$ and an additional generator in degree 1 . The ring $\mathbb{C}[\mathfrak{h}]^{\Gamma_{n}}$ is a polynomial algebra with generators in degrees $l, 2 l, \ldots, n l$. Hence

$$
\operatorname{ch}_{t} \mathbb{C}[\mathfrak{h}]^{\Gamma_{n-1}}=\frac{1}{1-t} \prod_{i=1}^{n-1} \frac{1}{1-t^{i l}}, \quad \mathbb{C}[\mathfrak{h}]^{\Gamma_{n}}=\prod_{i=1}^{n} \frac{1}{1-t^{i l}} .
$$

It follows that $\operatorname{ch}_{t} \mathbb{C}[\mathfrak{h}]^{\Gamma_{n-1}} /\left\langle\mathbb{C}[\mathfrak{h}]_{+}^{\Gamma_{n}}\right\rangle=\frac{\operatorname{ch}_{t} \mathbb{C}[\mathfrak{h}]^{\Gamma_{n-1}}}{\mathrm{ch}_{t} \mathbb{C}[\mathfrak{h}]^{\Gamma_{n}}}=\frac{1-t^{n l}}{1-t}=[n l]_{t}$. 
Definition 8.3. Suppose that we are given an $l$-multipartition $\underline{\lambda} \in \mathcal{P}(l, n)$ and the corresponding irreducible representation $S(\underline{\lambda})$ of $\Gamma_{n}$. We regard $S(\underline{\lambda})$ as a graded $\Gamma_{n}$-module concentrated in degree zero. The fake degree polynomial associated to $\underline{\lambda}$ is defined as

$$
f_{\underline{\lambda}}(t):=\sum_{k \in \mathbb{Z}}\left[\mathbb{C}[\mathfrak{h}]^{c o \Gamma_{n}}: S(\underline{\lambda})^{*}[k]\right] t^{k} .
$$

Theorem 8.4 ([35, Theorem 5.3]). Let $\underline{\lambda} \in \mathcal{P}(l, n)$. We have

$$
f_{\underline{\lambda}}(t)=t^{r(\underline{\lambda})}\left(t^{l}\right)_{n} \prod_{i=0}^{l-1} \frac{t^{l \cdot n\left(\lambda^{i}\right)}}{H_{\lambda^{i}}\left(t^{l}\right)}=t^{r(\underline{\lambda})}\left(t^{l}\right)_{n} \prod_{i=0}^{l-1} s_{\lambda^{i}}\left(1, t^{l}, t^{2 l}, \ldots\right) .
$$

In particular, if $\lambda$ is a partition of $n$ then $f_{\lambda}=(t)_{n} \frac{t^{n(\lambda)}}{H_{\lambda}(t)}=(t)_{n} s_{\lambda}\left(1, t, t^{2}, \ldots\right)$.

8.2. Auxiliary calculations. Fix $\underline{\lambda} \in \mathcal{P}(l, n)$. Let $q(\underline{\lambda})$ denote the degree in which the trivial $\Gamma_{n}$-module triv occurs in $L(\underline{\lambda})$.

Lemma 8.5. We have a graded $\mathbb{H}_{\mathbf{h}}$-module isomorphism

$$
\mathcal{R}_{\mathbf{h}, \underline{\lambda}}=\mathbb{H}_{\mathbf{h}} e_{n} \otimes_{e_{n} \mathbb{H}_{\mathbf{h}} e_{n}} e_{n} L_{\underline{\lambda}} \cong L(\underline{\lambda})[-q(\underline{\lambda})]
$$

and hence a graded vector space isomorphism

$$
\left(\mathcal{R}_{\mathbf{h}}^{\Gamma_{n-1}}\right)_{\underline{\lambda}}=e_{n-1} \mathcal{R}_{\mathbf{h}, \underline{\lambda}} \cong e_{n-1} L(\underline{\lambda})[-q(\underline{\lambda})] .
$$

Proof. As ungraded $\mathbb{H}_{\mathbf{h}}$-modules, $\mathcal{R}_{\mathbf{h}, \underline{\lambda}}$ and $L(\underline{\lambda})$ are clearly isomorphic. Since they are simple, one is a graded shift of the other. The trivial $\Gamma_{n}$-representation triv occurs in $L(\underline{\lambda})$ in degree $q(\underline{\lambda})$. On the other hand, we can identify triv with the subspace $e_{n} \otimes_{e_{n}} \mathbb{H}_{\mathbf{h}} e_{n} e_{n} L_{\underline{\lambda}}$ of $\mathcal{R}_{\mathbf{h}, \underline{\lambda}}$, so triv occurs in $\mathcal{R}_{\mathbf{h}, \underline{\lambda}}$ in degree zero.

Let us calculate the graded multiplicity of $L(\underline{\lambda})$ in $\Delta(\underline{\lambda})$.

Lemma 8.6. Let $\underline{\lambda} \in \mathcal{P}(l, n)$. The simple $\overline{\mathbb{H}}_{\mathbf{h}}$-module $L(\underline{\lambda})$ occurs in $\Delta(\underline{\lambda})$ with graded multiplicity

$$
\sum_{k \in \mathbb{Z}}[\Delta(\underline{\lambda}): L(\underline{\lambda})[k]] t^{k}=t^{-q(\underline{\lambda})} f_{\underline{\lambda}}(t) .
$$

Proof. By [1, Lemma 3.3], we have

Hence

$$
\operatorname{ch}_{t} L(\underline{\lambda})=\frac{t^{q(\underline{\lambda})} \operatorname{ch}_{t} \Delta(\underline{\lambda})}{f_{\underline{\lambda}}(t)} .
$$

$$
\sum_{k \in \mathbb{Z}}[\Delta(\underline{\lambda}): L(\underline{\lambda})[k]] t^{k}=\frac{\operatorname{ch}_{t} \Delta(\underline{\lambda})}{\operatorname{ch}_{t} L(\underline{\lambda})}=t^{-q(\underline{\lambda})} f_{\underline{\lambda}}(t) .
$$

Let us calculate the character of $e_{n-1} \Delta(\underline{\lambda})$.

Lemma 8.7. We have

$$
\operatorname{ch}_{t} e_{n-1} \Delta(\underline{\lambda})=[\ln ]_{t} \sum_{\underline{\mu} \uparrow \underline{\lambda}} f_{\underline{\mu}}(t) .
$$

Proof. By Lemma 8.2 and Proposition 2.1, we have isomorphisms of graded $\Gamma_{n-1}$-modules

$$
\begin{aligned}
\left.\Delta(\underline{\lambda})\right|_{\Gamma_{n-1}} & \left.\left.\cong \mathbb{C}[\mathfrak{h}]^{c o \Gamma_{n}}\right|_{\Gamma_{n-1}} \otimes S(\underline{\lambda})\right|_{\Gamma_{n-1}} \\
& \cong\left(\mathbb{C}\left[\mathfrak{h}^{\prime}\right]^{c o \Gamma_{n-1}} \otimes U\right) \otimes \bigoplus_{\underline{\mu} \uparrow \underline{\lambda}} S(\underline{\mu}) \cong \bigoplus_{\underline{\mu} \hat{\lambda}} \Delta(\underline{\mu}) \otimes U,
\end{aligned}
$$

where $U$ is a graded vector space with character $\operatorname{ch}_{t} U=[\ln ]_{t}$. Hence

$$
e_{n-1} \Delta(\underline{\lambda}) \cong \bigoplus_{\underline{\mu} \uparrow \underline{\lambda}} e_{n-1} \Delta(\underline{\mu}) \otimes U
$$

as graded $\Gamma_{n-1}$-modules. For each $\mu \uparrow \underline{\lambda}$, we have

$$
\operatorname{ch}_{t} e_{n-1} \Delta(\underline{\mu})=\sum_{k \in \mathbb{Z}}[\Delta(\underline{\mu}): \operatorname{triv}[k]] t^{k}=f_{\underline{\mu}}(t) .
$$

The first equality above is obvious, for the second see, e.g., the proof of [15. Theorem 5.6]. Combining (36) with (37) we obtain (35). 
8.3. The character of the fibre. We can now put our calculations together to obtain the character of $\left(\mathcal{R}_{\mathbf{h}}^{\Gamma_{n-1}}\right)_{\underline{\lambda}}$.

Theorem 8.8. Let $\underline{\lambda} \in \mathcal{P}(l, n)$. Then

$$
\operatorname{ch}_{t}\left(\mathcal{R}_{\mathbf{h}}^{\Gamma_{n-1}}\right)_{\underline{\lambda}}=[\ln ]_{t} \sum_{\underline{\mu} \uparrow \underline{\underline{\lambda}}} \frac{f_{\underline{\mu}}(t)}{f_{\underline{\lambda}}(t)} .
$$

Proof. By Lemmas 8.5 8.6 and 8.7 , we have

$$
\begin{aligned}
\operatorname{ch}_{t}\left(\mathcal{R}_{\mathbf{h}}^{\Gamma_{n-1}}\right)_{\underline{\lambda}} & =t^{-q(\underline{\lambda})} \cdot \operatorname{ch}_{t} e_{n-1} L(\underline{\lambda})=\left(t^{-q(\underline{\lambda})} \cdot \operatorname{ch}_{t} e_{n-1} \Delta(\underline{\lambda})\right) /\left(t^{-q(\underline{\lambda})} f_{\underline{\lambda}}(t)\right) \\
& =\operatorname{ch}_{t} e_{n-1} \Delta(\underline{\lambda}) / f_{\underline{\lambda}}(t)=[\ln ]_{t} \sum_{\underline{\mu} \uparrow \underline{\hat{\lambda}}} \frac{f_{\underline{\mu}}(t)}{f_{\underline{\lambda}}(t)} .
\end{aligned}
$$

Corollary 8.9. We have

$$
\operatorname{ch}_{t}\left(\mathcal{R}_{\mathbf{h}}^{\Gamma_{n-1}}\right)_{\underline{\lambda}}=\frac{1}{1-t} \sum_{i=0}^{l-1} t^{-i} \sum_{\substack{\mu \uparrow \underline{\lambda}, \mu^{i} \neq \lambda^{i}}} \frac{s_{\mu^{i}}\left(1, t^{l}, t^{2 l}, \ldots\right)}{s_{\lambda^{i}}\left(1, t^{l}, t^{2 l}, \ldots\right)}=\frac{1}{1-t} \sum_{i=0}^{l-1} t^{-i} \sum_{\substack { \mu \uparrow \\
\begin{subarray}{c}{\mu \\
\mu^{i} \neq \lambda^{i}{ \mu \uparrow \\
\begin{subarray} { c } { \mu \\
\mu ^ { i } \neq \lambda ^ { i } } }\end{subarray}} \frac{t^{l \cdot n\left(\mu^{i}\right)} H_{\lambda^{i}}\left(t^{l}\right)}{t^{l \cdot n\left(\lambda^{i}\right)} H_{\mu^{i}}\left(t^{l}\right)} .
$$

In particular, if $l=1$ then $\operatorname{ch}_{t}\left(\mathcal{R}_{\mathbf{h}}^{\Gamma_{n-1}}\right)_{\lambda}=\frac{1}{1-t} \sum_{\mu \uparrow \lambda} \frac{s_{\mu}\left(1, t, t^{2}, \ldots\right)}{s_{\lambda}\left(1, t, t^{2}, \ldots\right)}=\frac{1}{1-t} \sum_{\mu \uparrow \lambda} \frac{t^{n(\mu)}}{t^{n(\lambda)}} \frac{H_{\lambda}(t)}{H_{\mu}(t)}$.

Proof. This follows immediately from Theorems 8.4 and 8.8

We can now prove Theorem 8.1 .

Proof of Theorem 8.1. Choose any parameter $\mathbf{h} \in \mathbb{Q}^{l}$ such that Spec $Z_{\mathbf{h}}$ is smooth. By Theorem 3.3 the Etingof-Ginzburg map induces an isomorphism of vector bundles $\mathcal{R}_{\mathbf{h}}^{\Gamma_{n-1}} \cong$ $\mathcal{V}_{\mathbf{h}}$. Since the Etingof-Ginzburg map is $\mathbb{C}^{*}$-equivariant, we have

$$
\operatorname{ch}_{t}\left(\mathcal{V}_{\mathbf{h}}\right)_{\mu}=\operatorname{ch}_{t}\left(\mathcal{R}_{\mathbf{h}}^{\Gamma_{n-1}}\right)_{\text {Quot }(\mu)^{b}},
$$

by Theorem 7.18. Proposition 5.15 yields the formula for the LHS of 38 while Theorem 8.8 yields the formula for the RHS.

\section{9. $\mathbb{C}^{*}$-FIXED POINTS UNDER REFLECTION FUNCTORS}

Assume from now on that $l>1$. In this section we compute the bijections between $\mathbb{C}^{*}$-fixed points induced by reflection functors (see $\$ 4.9$ ).

Fix $\nu \in \odot(l)$ and let $\mathbf{d}_{\nu}=\left(d_{0}, \ldots, d_{l-1}\right)$ be the corresponding dimension vector (see 4.7). Assume that $\theta \in \mathbb{Q}^{l}$ is chosen so that $\theta_{i} \neq 0$ and the quiver variety $\mathcal{X}_{\theta}\left(n \delta+\mathbf{d}_{\nu}\right)$ is smooth. The reflection functor

$$
\mathfrak{R}_{i}: \mathcal{X}_{\theta}\left(n \delta+\mathbf{d}_{\nu}\right) \rightarrow \mathcal{X}_{\sigma_{i} \cdot \theta}\left(n \delta+\mathbf{d}_{\sigma_{i} * \nu}\right)
$$

is a $\mathbb{C}^{*}$-equivariant isomorphism and hence induces a bijection $\mathcal{X}_{\theta}\left(n \delta+\mathbf{d}_{\nu}\right)^{\mathbb{C}^{*}} \longleftrightarrow$ $\mathcal{X}_{\sigma_{i} \cdot \theta}\left(n \delta+\mathbf{d}_{\sigma_{i} * \nu}\right)^{\mathbb{C}^{*}}$ between the $\mathbb{C}^{*}$-fixed points. Composing it with the bijections from Corollary 5.16, we obtain a bijection

$$
\mathbf{R}_{i}: \mathcal{P}_{\nu^{t}}\left(n l+\left|\nu^{t}\right|\right) \rightarrow \mathcal{P}_{\left(\sigma_{i} * \nu\right)^{t}}\left(n l+\left|\left(\sigma_{i} * \nu\right)^{t}\right|\right) .
$$

Fix $\mu \in \mathcal{P}_{\nu^{t}}\left(n l+\left|\nu^{t}\right|\right)$. We are going to show that

$$
\mathbf{R}_{i}(\mu)=\left(\mathbf{T}_{i}\left(\mu^{t}\right)\right)^{t},
$$

where $\mathbf{T}_{i}\left(\mu^{t}\right)$ is the partition obtained from $\mu^{t}$ by adding all $i$-addable and removing all $i$-removable cells relative to $\mu^{t}$, as in Definition 4.9 .

9.1. The strategy. Our goal is to describe the action of reflection functors on the $\mathbb{C}^{*}$ fixed points combinatorially. In $\$ 9.2$ we endow the vector space $\widehat{\mathbf{V}}^{\nu}$ with a $\mathbb{Z}$-grading, which we call the " $\mu$-grading". A $\mathbb{C}^{*}$-fixed point is characterized uniquely by this grading. In $\$ 9.4$ we compute the $\mathbf{R}_{i}(\mu)$-grading on the vector space $\widehat{\mathbf{V}}^{\sigma_{i * \nu}}$. In $\$ 9.5$ and $\$ 9.6$ we use this calculation to give a combinatorial description of the partition $\mathbf{R}_{i}(\mu)$. 
9.2. The $\mu$-grading. Fix a $\mathbb{Z} / l \mathbb{Z}$-graded complex vector space $\widehat{\mathbf{V}}^{\nu}:=\bigoplus_{i=0}^{l-1} \mathbf{V}_{i}^{\nu}$ with $\operatorname{dim} \mathbf{V}_{i}^{\nu}=n+d_{i}$. Set $\mathbf{V}^{\nu}=\widehat{\mathbf{V}}^{\nu} \oplus \mathbf{V}_{\infty}$ with $\operatorname{dim} \mathbf{V}_{\infty}=1$. We are now going to introduce a $\mathbb{Z}$-grading on $\widehat{\mathbf{V}}^{\nu}$ which "lifts" the $\mathbb{Z} / l \mathbb{Z}$-grading.

Definition 9.1. We call a $\mathbb{Z}$-grading $\widehat{\mathbf{V}}^{\nu}=\bigoplus_{i \in \mathbb{Z}} \mathbf{W}_{i}$ a $\mu$-grading if it satisfies the following condition:

(C): for each $i \in \mathbb{Z}$ we have

$$
A(\mu)\left(\mathbf{W}_{i}\right) \subseteq \mathbf{W}_{i-1}, \quad \Lambda(\mu)\left(\mathbf{W}_{i}\right) \subseteq \mathbf{W}_{i+1}, \quad J(\mu)\left(\mathbf{V}_{\infty}\right) \subseteq \mathbf{W}_{0}, \quad I(\mu)\left(\mathbf{W}_{0}\right)=\mathbf{V}_{\infty}
$$

where $A(\mu), \Lambda(\mu), J(\mu)$ and $I(\mu)$ are as in Definition 5.6 .

Proposition 9.2. A $\mu$-grading on $\widehat{\mathbf{V}}^{\nu}$ exists and is unique.

Proof. We first prove existence. Let $\mu=\left(a_{1}, \ldots, a_{k} \mid b_{1}, \ldots, b_{k}\right)$ be the Frobenius form of $\mu$. Set $r_{i}=b_{i}+1, m_{i}=a_{i}+b_{i}+1$ and $q_{i}=\sum_{j<i} m_{j}+r_{i}$ for $1 \leq i \leq k$. Recall the ordered basis $\{\operatorname{Bas}(i)|1 \leq i \leq n l+| \nu \mid\}$ of $\widehat{\mathbf{V}}^{\nu}$ from $\$ 5.3$. We now define $\mathbf{W}_{j}$ by the rule that for each $1 \leq i \leq k$ :

$$
\operatorname{Bas}\left(q_{i}-j\right) \in \mathbf{W}_{j} \quad\left(0 \leq j \leq b_{i}\right), \quad \operatorname{Bas}\left(q_{i}+j\right) \in \mathbf{W}_{-j} \quad\left(1 \leq j \leq a_{i}\right) .
$$

It follows directly from the construction of the matrices $A(\mu), \Lambda(\mu), J(\mu)$ and $I(\mu)$ that this grading satisfies condition $(\mathbf{C})$ in Definition 9.1. Hence it is a $\mu$-grading. Finally we observe that the definition of $\Lambda(\mu)$ and Lemma 5.10 imply that

$$
\mathbf{W}_{j}=(A(\mu))^{j}\left(\mathbf{W}_{0}\right) \quad(j<0), \quad \mathbf{W}_{j}=(\Lambda(\mu))^{j}\left(\mathbf{W}_{0}\right) \quad(j>0) .
$$

We now prove uniqueness. Let $\widehat{\mathbf{V}}^{\nu}=\bigoplus_{i \in \mathbb{Z}} \mathbf{W}_{i}^{\prime}$ be a $\mu$-grading. Choose $0 \neq v_{\infty} \in \mathbf{V}_{\infty}$. Then by the definition of $J(\mu)$ for a generic parameter $\theta$ there exist $c_{1}, \ldots, c_{k} \in \mathbb{C}^{*}$ such that $u_{1}:=c_{1} \operatorname{Bas}\left(q_{1}\right)+\ldots+c_{k} \operatorname{Bas}\left(q_{k}\right)=J(\mu)\left(v_{\infty}\right) \in \mathbf{W}_{0}^{\prime}$. Lemma 5.10 together with the fact that the parameter $\theta$ is generic implies that $0 \neq(A(\mu))^{a_{1}}\left(\operatorname{Bas}\left(q_{1}\right)\right)=t(A(\mu))^{a_{1}}\left(u_{1}\right)$ for some scalar $t \in \mathbb{C}^{*}$. Since $u_{1} \in \mathbf{W}_{0}^{\prime}$ and the operator $A(\mu)$ lowers degree by one, we have $0 \neq(A(\mu))^{a_{1}}\left(\operatorname{Bas}\left(q_{1}\right)\right) \in \mathbf{W}_{-a_{1}}^{\prime}$. It now follows from the definition of the matrices $A(\mu)$ and $\Lambda(\mu)$ and the genericity of $\theta$ that $(\Lambda(\mu))^{a_{1}} \circ(A(\mu))^{a_{1}}\left(u_{1}\right)=t^{\prime} \operatorname{Bas}\left(q_{1}\right)$ for some scalar $t^{\prime} \in \mathbb{C}^{*}$. Since the operator $\Lambda(\mu)$ raises degree by one, we have $\operatorname{Bas}\left(q_{1}\right), c_{2} \operatorname{Bas}\left(q_{2}\right)+$ $\ldots+c_{k} \operatorname{Bas}\left(q_{k}\right) \in \mathbf{W}_{0}^{\prime}$.

We can now apply an analogous argument to the vectors $\operatorname{Bas}\left(q_{2}\right)$ and $u_{2}:=c_{2} \operatorname{Bas}\left(q_{2}\right)+$ $\ldots+c_{k} \operatorname{Bas}\left(q_{k}\right)$. By Lemma 5.10 we have $0 \neq(A(\mu))^{a_{2}}\left(\operatorname{Bas}\left(q_{2}\right)\right)=t(A(\mu))^{a_{2}}\left(u_{2}\right)+$ $t^{\prime}(A(\mu))^{a_{2}}\left(\operatorname{Bas}\left(q_{1}\right)\right)$ for some scalars $t, t^{\prime} \in \mathbb{C}^{*}$. Since $u_{2}$ and $\operatorname{Bas}\left(q_{1}\right)$ are homogeneous elements of degree zero and $A(\mu)$ lowers degree by one, we get $0 \neq(A(\mu))^{a_{2}}\left(\operatorname{Bas}\left(q_{2}\right)\right) \in$ $\mathbf{W}_{-a_{2}}^{\prime}$. Moreover, Lemma 5.10 implies that $(A(\mu))^{a_{2}}\left(\operatorname{Bas}\left(q_{2}\right)\right)$ is a linear combination of $\operatorname{Bas}\left(q_{1}+a_{2}\right)$ and $\operatorname{Bas}\left(q_{2}+a_{2}\right)$. But $\operatorname{Bas}\left(q_{1}+a_{2}\right)=(A(\mu))^{a_{2}}\left(\operatorname{Bas}\left(q_{1}\right)\right)$ up to multiplication by a non-zero scalar, so $\operatorname{Bas}\left(q_{1}+a_{2}\right) \in \mathbf{W}_{-a_{2}}^{\prime}$. Hence $\operatorname{Bas}\left(q_{2}+a_{2}\right) \in \mathbf{W}_{-a_{2}}^{\prime}$ and so $\operatorname{Bas}\left(q_{2}\right)=(\Lambda(\mu))^{a_{2}}\left(\operatorname{Bas}\left(q_{2}+a_{2}\right)\right) \in \mathbf{W}_{0}^{\prime}$. We conclude that $\operatorname{Bas}\left(q_{2}\right), u_{3}:=c_{3} \operatorname{Bas}\left(q_{3}\right)+$ $\ldots+c_{k} \operatorname{Bas}\left(q_{k}\right) \in \mathbf{W}_{0}^{\prime}$. Repeating this argument sufficiently many times shows that $\operatorname{Bas}\left(q_{1}\right), \ldots, \operatorname{Bas}\left(q_{k}\right) \in \mathbf{W}_{0}^{\prime}$. It follows that $\mathbf{W}_{0}^{\prime}=\mathbf{W}_{0}$. Condition $(\mathbf{C})$ and 42 now imply that $\mathbf{W}_{i}^{\prime}=\mathbf{W}_{i}$ for all $i \in \mathbb{Z}$.

Thanks to Proposition 9.2 , we can talk about the $\mu$-grading on $\widehat{\mathbf{V}}^{\nu}$. Let us denote it by $\widehat{\mathbf{V}}^{\nu}=\bigoplus_{i \in \mathbb{Z}} \mathbf{W}_{i}^{\mu}$. We write $\operatorname{deg}_{\mu} v=i$ if $v \in \mathbf{W}_{i}^{\mu}$. Moreover, let $P_{\mu}:=\sum_{i \in \mathbb{Z}} \operatorname{dim} \mathbf{W}_{i}^{\mu} t^{i}$ denote the Poincaré polynomial of $\mathbf{V}^{\nu}$ with respect to the $\mu$-grading. Consider the function

$$
\mathcal{X}_{\theta}\left(n \delta+\mathbf{d}_{\nu}\right)^{\mathbb{C}^{*}} \rightarrow \mathbb{Z}\left[t, t^{-1}\right], \quad[\mathbf{A}(\mu)] \mapsto P_{\mu} .
$$

Proposition 9.3. We have $P_{\mu}=\operatorname{Res}_{\mu^{t}}(t)$. Moreover, the function (43) is injective.

Proof. Fix $j \geq 0$. By 41], $\operatorname{Bas}\left(q_{i}-j\right) \in \mathbf{W}_{j}^{\mu}$ if and only if $j \leq b_{i}$, for $i=1, \ldots, k$. Moreover, $\left\{\operatorname{Bas}\left(q_{i}-j\right) \mid j \leq b_{i}\right\}$ form a basis of $\mathbf{W}_{j}^{\mu}$. Hence $\operatorname{dim} \mathbf{W}_{j}^{\mu}=\sum_{i=1}^{k} \mathbf{1}_{j \leq b_{i}}$, where $\mathbf{1}_{j \leq b_{i}}$ is the indicator function taking value 1 if $j \leq b_{i}$ and 0 otherwise. But $\sum_{i=1}^{k} \mathbf{1}_{j \leq b_{i}}$ is precisely the number of cells of content $-j$ in $\mu$, which is the same as the number of cells of content $j$ in $\mu^{t}$. The argument for $j<0$ is analogous. This proves the first claim. The second claim follows immediately from the fact that partitions are determined uniquely by their residues. 
Suppose that we are given a $\mathbb{C}^{*}$-fixed point and want to find out which partition it is labelled by. Proposition 9.3 implies that to do so we only need to compute the $\mathbb{Z}$-grading on $\widehat{\mathbf{V}}^{\nu}$ associated to the fixed point and the corresponding Poincaré polynomial.

Lemma 9.4. The following hold:

a) The restricted maps

$$
A(\mu): \mathbf{W}_{i}^{\mu} \rightarrow \mathbf{W}_{i-1}^{\mu}(i \leq 0), \quad \Lambda(\mu): \mathbf{W}_{i}^{\mu} \rightarrow \mathbf{W}_{i+1}^{\mu},(i \geq 0)
$$

are surjective.

b) The restricted maps

$$
A(\mu): \mathbf{W}_{i}^{\mu} \rightarrow \mathbf{W}_{i-1}^{\mu}(i>0), \quad \Lambda(\mu): \mathbf{W}_{i}^{\mu} \rightarrow \mathbf{W}_{i+1}^{\mu}(i<0)
$$

are injective.

c) We have $\mathbf{V}_{j}^{\nu}=\bigoplus_{i \in \mathbb{Z}} \mathbf{W}_{j+i l}^{\mu}$ for each $j=0, \ldots, l-1$.

Proof. The first claim is just a restatement of 42 . The second claim follows directly from Lemma 5.10 The third claim follows from 42 and the fact that $A(\mu)(\operatorname{resp} . \Lambda(\mu))$ is a homogeneous operator of degree -1 (resp. 1) with respect to the $\mathbb{Z} / l \mathbb{Z}$-grading on $\widehat{\mathbf{V}}^{\nu}$.

Remark 9.5. We may interpret Lemma 9.4(c) as saying that the $\mu$-grading on $\widehat{\mathbf{V}}^{\nu}$ is a lift of the $\mathbb{Z} / l \mathbb{Z}$-grading. Furthermore, we may think of the $\mu$-grading as the grading on the representation of the $A_{\infty}$-quiver corresponding to the fixed point $[\mathbf{A}(\mu)]$. More details about the connection between quiver varieties of type $A_{\infty}$ and the $\mathbb{C}^{*}$-fixed points in cyclic quiver varieties can be found in [34, §4].

9.3. Explicit definition of reflection functors. Let us recall the explicit definition of reflection functors from [30, Proposition 3.19] and [8, §5]. Fix a $\mathbb{Z} / l \mathbb{Z}$-graded complex vector space $\widehat{\mathbf{V}}^{\sigma_{i} * \nu}:=\bigoplus_{j=0}^{l-1} \mathbf{V}_{j}^{\sigma_{i} * \nu}$ with $\operatorname{dim} \mathbf{V}_{j}^{\sigma_{i} * \nu}=n+d_{j}^{\prime}$. Set $\mathbf{V}^{\sigma_{i} * \nu}:=\widehat{\mathbf{V}}^{\sigma_{i} * \nu} \oplus \mathbf{V}_{\infty}$. To simplify notation, set $\mathbf{d}:=\mathbf{d}_{\nu}$ and $\mathbf{d}^{\prime}:=\sigma_{i} * \mathbf{d}_{\nu}$.

Let us first define a map $\mu_{n \delta+\mathbf{d}}^{-1}(\theta) \rightarrow \mu_{n \delta+\mathbf{d}^{\prime}}^{-1}(\sigma \cdot \theta)$ lifting (39), which we also denote by $\mathfrak{R}_{i}$. Let $\rho=(\mathbf{X}, \mathbf{Y}, I, J) \in \mu_{n \delta+\mathbf{d}}^{-1}(\theta)$. The reflected quiver representation

$$
\mathfrak{R}_{i}(\rho)=:\left(\mathbf{X}^{\prime}, \mathbf{Y}^{\prime}, I^{\prime}, J^{\prime}\right)
$$

is defined as follows. Suppose that $i \neq 0$. We have maps

$$
\mathbf{V}_{i}^{\nu} \stackrel{Y_{i}-X_{i}}{\longrightarrow} \mathbf{V}_{i-1}^{\nu} \oplus \mathbf{V}_{i+1}^{\nu} \stackrel{X_{i-1}+Y_{i+1}}{\longrightarrow} \mathbf{V}_{i}^{\nu}
$$

Set $\psi_{i}:=Y_{i}-X_{i}$ and $\phi_{i}:=X_{i-1}+Y_{i+1}$ (the indices should be considered mod $l$ ). The preprojective relations (i.e. the relations defining the fibre $\mu_{n \delta+\mathbf{d}}^{-1}(\theta)$ ) ensure that we have a splitting $\mathbf{V}_{i-1}^{\nu} \oplus \mathbf{V}_{i+1}^{\nu}=\operatorname{Im} \psi_{i} \oplus \operatorname{ker} \phi_{i}$. The underlying vector space of the quiver representation $\mathfrak{R}_{i}(\rho)$ is obtained from $\mathbf{V}^{\nu}$ by replacing $\mathbf{V}_{i}^{\nu}$ with ker $\phi_{i}$. We have an isomorphism of vector spaces $\mathbf{V}^{\sigma_{i} * \nu} \cong \operatorname{ker} \phi_{i} \oplus \bigoplus_{j \neq i} \mathbf{V}_{j}^{\nu} \oplus \mathbf{V}_{\infty}$ preserving the quiver grading. Let us define $\mathfrak{R}_{i}(\rho)$. We have $X_{j}^{\prime}=X_{j}$ unless $j \in\{i-1, i\}$. We also have $Y_{j}^{\prime}=Y_{j}$ unless $j \in\{i, i+1\}$. Set $I^{\prime}=I$ and $J^{\prime}=J$. The maps $X_{i}^{\prime}$ and $Y_{i}^{\prime}$ are defined as the composite maps

$$
\begin{aligned}
& X_{i}^{\prime}: \operatorname{ker} \phi_{i} \hookrightarrow \operatorname{ker} \phi_{i} \oplus \operatorname{Im} \psi_{i}=\mathbf{V}_{i-1}^{\nu} \oplus \mathbf{V}_{i+1}^{\nu} \rightarrow \mathbf{V}_{i+1}^{\nu}, \\
& Y_{i}^{\prime}: \operatorname{ker} \phi_{i} \hookrightarrow \operatorname{ker} \phi_{i} \oplus \operatorname{Im} \psi_{i}=\mathbf{V}_{i-1}^{\nu} \oplus \mathbf{V}_{i+1}^{\nu} \rightarrow \mathbf{V}_{i-1}^{\nu} .
\end{aligned}
$$

The maps $X_{i-1}^{\prime}$ and $Y_{i+1}^{\prime}$ are defined as the composite maps

$$
\begin{aligned}
X_{i-1}^{\prime}: \mathbf{V}_{i-1}^{\nu} \stackrel{\cdot\left(-\theta_{i}\right)}{\longrightarrow} \mathbf{V}_{i-1}^{\nu} \hookrightarrow \mathbf{V}_{i-1}^{\nu} \oplus \mathbf{V}_{i+1}^{\nu}=\operatorname{ker} \phi_{i} \oplus \operatorname{Im} \psi_{i} \rightarrow \operatorname{ker} \phi_{i}, \\
Y_{i+1}^{\prime}: \mathbf{V}_{i+1}^{\nu} \stackrel{\cdot\left(-\theta_{i}\right)}{\longrightarrow} \mathbf{V}_{i+1}^{\nu} \hookrightarrow \mathbf{V}_{i-1}^{\nu} \oplus \mathbf{V}_{i+1}^{\nu}=\operatorname{ker} \phi_{i} \oplus \operatorname{Im} \psi_{i} \rightarrow \operatorname{ker} \phi_{i} \stackrel{\cdot(-1)}{\longrightarrow} \operatorname{ker} \phi_{i} .
\end{aligned}
$$

The minus signs before $X_{i}$ in (44) as well as in the last arrow above come from the fact that our quiver does not have a sink at the vertex $i$ as in [8, $\S 5]$ - hence the need for these adjustments. If $i=0$ one defines $\mathfrak{R}_{i}(\rho)$ analogously using maps

$$
\mathbf{V}_{0}^{\nu} \stackrel{\psi_{0}}{\longrightarrow} \mathbf{V}_{l-1}^{\nu} \oplus \mathbf{V}_{1}^{\nu} \oplus \mathbf{V}_{\infty} \stackrel{\phi_{0}}{\longrightarrow} \mathbf{V}_{0}^{\nu}
$$

with $\psi_{0}=Y_{0}-X_{0}+I$ and $\phi_{0}=X_{l-1}+Y_{1}+J$. 
9.4. The reflected grading. Let us apply the definitions from $\$ 9.3$ to $\rho=\mathbf{A}(\mu)$. More specifically, for $i=0, \ldots, l-1$, we set $X_{i}=\left.\Lambda(\mu)\right|_{\mathbf{v}_{i}^{\nu}}, Y_{i}=\left.A(\mu)\right|_{\mathbf{v}_{i}^{\nu}}, I=I(\mu)$ and $J=J(\mu)$. The reflected quiver representation $\mathfrak{R}_{i}(\mathbf{A}(\mu)) \in \mu_{n \delta+\mathbf{d}^{\prime}}^{-1}(\sigma \cdot \theta)$ is conjugate under the $G\left(n \delta+\mathbf{d}^{\prime}\right)$-action to $\mathbf{A}\left(\mathbf{R}_{i}(\mu)\right)$. We now compute the $\mathbf{R}_{i}(\mu)$-grading on $\widehat{\mathbf{V}}^{\sigma_{i} * \nu}$.

We have direct sum decompositions

$$
\psi_{i}:=\bigoplus_{j \in \mathbb{Z}} \psi_{i}^{j}, \quad \phi_{i}:=\bigoplus_{j \in \mathbb{Z}} \phi_{i}^{j},
$$

with $\psi_{i}^{j}=\left.\psi_{i}\right|_{\mathbf{W}_{j l+i}^{\mu}}$ and $\phi_{i}^{j}=\left.\phi_{i}\right|_{\mathbf{W}_{j l+i-1}^{\mu} \oplus \mathbf{W}_{j l+i+1}^{\mu}}(i \neq 0$ or $j \neq 0)$ and $\psi_{0}^{0}:=\left.\psi_{0}\right|_{\mathbf{W}_{0}^{\mu}}$. Hence $\mathbf{V}_{i}^{\sigma_{i} * \nu}=\operatorname{ker} \phi_{i}=\bigoplus_{j \in \mathbb{Z}} \operatorname{ker} \phi_{i}^{j}$. Moreover, (44) and 47) decompose as direct sums of maps

$$
\begin{aligned}
& \mathbf{W}_{j l+i}^{\mu} \stackrel{\psi_{i}^{j}}{\longrightarrow} \mathbf{W}_{j l+i-1}^{\mu} \oplus \mathbf{W}_{j l+i+1}^{\mu} \stackrel{\phi_{i}^{j}}{\longrightarrow} \mathbf{W}_{j l+i}^{\mu} \quad(j \neq 0 \text { or } i \neq 0) . \\
& \mathbf{W}_{0}^{\mu} \stackrel{\psi_{0}^{0}}{\longrightarrow} \mathbf{W}_{-1}^{\mu} \oplus \mathbf{W}_{1}^{\mu} \oplus \mathbf{V}_{\infty} \stackrel{\phi_{0}^{0}}{\longrightarrow} \mathbf{W}_{0}^{\mu} .
\end{aligned}
$$

These direct sum decompositions together with the preprojective relations imply the following lemma.

Lemma 9.6. Let $i \in\{0, \ldots, l-1\}$ and $j \in \mathbb{Z}$. If $i=0$ and $j=0$ then $\operatorname{Im} \psi_{0}^{0} \oplus \operatorname{ker} \phi_{0}^{0}=$ $\mathbf{W}_{-1}^{\mu} \oplus \mathbf{W}_{1}^{\mu} \oplus \mathbf{V}_{\infty}$. Otherwise $\operatorname{Im} \psi_{i}^{j} \oplus \operatorname{ker} \phi_{i}^{j}=\mathbf{W}_{j l+i-1}^{\mu} \oplus \mathbf{W}_{j l+i+1}^{\mu}$.

Define

$$
\mathbf{U}_{j}:=\mathbf{W}_{j}^{\mu} \quad(j \neq i \bmod l), \quad \mathbf{U}_{l j+i}:=\operatorname{ker} \phi_{i}^{j} \quad(j \in \mathbb{Z}) .
$$

Proposition 9.7. The $\mathbb{Z}$-grading $\widehat{\mathbf{V}}^{\sigma_{i} * \nu}=\bigoplus_{j \in \mathbb{Z}} \mathbf{U}_{j}$ is the $\mathbf{R}_{i}(\mu)$-grading on $\widehat{\mathbf{V}}^{\sigma_{i} * \nu}$.

Proof. It suffices to show that $\widehat{\mathbf{V}}^{\sigma_{i} * \nu}=\bigoplus_{j \in \mathbb{Z}} \mathbf{U}_{j}$ satisfies condition $(\mathbf{C})$ in Definition 9.1 Suppose $i \neq 0$. Then for each $j \in \mathbb{Z}$ we need to check that

$$
\begin{array}{ll}
A\left(\mathbf{R}_{i}(\mu)\right)\left(\mathbf{U}_{j l+i+1}\right) \subseteq \mathbf{U}_{j l+i}, & \Lambda\left(\mathbf{R}_{i}(\mu)\right)\left(\mathbf{U}_{j l+i-1}\right) \subseteq \mathbf{U}_{j l+i}, \\
A\left(\mathbf{R}_{i}(\mu)\right)\left(\mathbf{U}_{j l+i}\right) \subseteq \mathbf{U}_{j l+i-1}, & \Lambda\left(\mathbf{R}_{i}(\mu)\right)\left(\mathbf{U}_{j l+i}\right) \subseteq \mathbf{U}_{j l+i+1} .
\end{array}
$$

If $i=0$ we additionally need to check that $I\left(\mathbf{R}_{i}(\mu)\right)\left(\mathbf{U}_{0}\right)=\mathbf{V}_{\infty}$ and $J\left(\mathbf{R}_{i}(\mu)\right)\left(\mathbf{V}_{\infty}\right) \subseteq \mathbf{U}_{0}$.

All of the inclusions above follow directly from Lemma 9.6 and the definition of reflection functors. For example, let us assume that $i \neq 0$ or $j \neq 0$ and consider the inclusion $A\left(\mathbf{R}_{i}(\mu)\right)\left(\mathbf{U}_{j l+i+1}\right) \subseteq \mathbf{U}_{j l+i}:=\operatorname{ker} \phi_{i}^{j}$. The map $A\left(\mathbf{R}_{i}(\mu)\right): \mathbf{V}_{i+1}^{\sigma_{i} * \nu}=\mathbf{V}_{i+1}^{\nu} \rightarrow \mathbf{V}_{i}^{\sigma_{i} * \nu}=$ $\operatorname{ker} \phi_{i}$ is given by (46). Consider its restriction to the subspace $\mathbf{U}_{j l+i+1}=\mathbf{W}_{j l+i+1}^{\mu} \subseteq$ $\mathbf{V}_{i+1}^{\nu}$. By Lemma 9.6 , we have $\mathbf{W}_{j l+i+1}^{\mu} \subseteq \mathbf{W}_{j l+i-1}^{\mu} \oplus \mathbf{W}_{j l+i+1}^{\mu}=\operatorname{ker} \phi_{i}^{j} \oplus \operatorname{Im} \psi_{i}^{j}$. Hence $A\left(\mathbf{R}_{i}(\mu)\right)$, restricted to $\mathbf{W}_{j l+i+1}^{\mu}$, is the composition

$\mathbf{W}_{j l+i+1}^{\mu} \stackrel{\cdot\left(-\theta_{i}\right)}{\longrightarrow} \mathbf{W}_{j l+i+1}^{\mu} \hookrightarrow \mathbf{W}_{j l+i-1}^{\mu} \oplus \mathbf{W}_{j l+i+1}^{\mu}=\operatorname{ker} \phi_{i}^{j} \oplus \operatorname{Im} \psi_{i}^{j} \rightarrow \operatorname{ker} \phi_{i}^{j} \stackrel{\cdot(-1)}{\longrightarrow} \operatorname{ker} \phi_{i}^{j}$.

In particular, $A\left(\mathbf{R}_{i}(\mu)\right)\left(\mathbf{W}_{j l+i+1}^{\mu}\right) \subseteq \operatorname{ker} \phi_{i}^{j}$, as desired. The other inclusions are proven analogously.

We have described the $\mathbf{R}_{i}(\mu)$-grading on $\widehat{\mathbf{V}}^{\sigma_{i} * \nu}$. We are now going to compute the corresponding Poincaré polynomial $P_{\mathbf{R}_{i}(\mu)}$.

Lemma 9.8. Let $\widehat{\mathbf{V}}^{\nu}=\bigoplus_{i \in \mathbb{Z}} \mathbf{W}_{i}^{\mu}$ be the $\mu$-grading on $\widehat{\mathbf{V}}^{\nu}$. Then

$$
\operatorname{dim} \operatorname{ker} \phi_{i}^{j}=\operatorname{dim} \mathbf{W}_{l j+i+1}^{\mu}+\operatorname{dim} \mathbf{W}_{l j+i-1}^{\mu}-\operatorname{dim} \mathbf{W}_{l j+i}^{\mu}
$$

if $j \neq 0$ or $i \neq 0$. Otherwise

$$
\operatorname{dim} \operatorname{ker} \phi_{0}^{0}=\operatorname{dim} \mathbf{W}_{1}^{\mu}+\operatorname{dim} \mathbf{W}_{-1}^{\mu}-\operatorname{dim} \mathbf{W}_{0}^{\mu}+1 .
$$

Proof. Assume that $j \neq 0$ or $i \neq 0$. Recall that $\psi_{i}^{j}=\left.A(\mu)\right|_{\mathbf{W}_{j l+i}^{\mu}}-\left.\Lambda(\mu)\right|_{\mathbf{W}_{j l+i}^{\mu}}$. Lemma 9.4 implies that either $\left.A(\mu)\right|_{\mathbf{W}_{j l+i}^{\mu}}$ or $\left.\Lambda(\mu)\right|_{\mathbf{W}_{j l+i}^{\mu}}$ is injective. Hence $\psi_{i}^{j}$ is injective. Therefore we have $\operatorname{dim} \operatorname{Im} \psi_{i}^{j}=\operatorname{dim} \mathbf{W}_{l j+i}^{\mu}$. The equality $\operatorname{dim} \operatorname{ker} \phi_{i}^{j}=\operatorname{dim} \mathbf{W}_{l j+i+1}^{\mu}+\operatorname{dim} \mathbf{W}_{l j+i-1}^{\mu}-$ $\operatorname{dim} \operatorname{Im} \psi_{i}^{j}$ now implies the lemma. The case $i=j=0$ is similar.

Write $P_{\mu}=\sum_{j \in \mathbb{Z}} a_{j}^{\mu} t^{j}$ with $a_{j}^{\mu}=\operatorname{dim} \mathbf{W}_{j}^{\mu}$ and $P_{\mathbf{R}_{i}(\mu)}=\sum_{j \in \mathbb{Z}} a_{j}^{\mathbf{R}_{i}(\mu)} t^{j}$ with $a_{j}^{\mathbf{R}_{i}(\mu)}=$ $\operatorname{dim} \mathbf{W}_{j}^{\mathbf{R}_{i}(\mu)}$. Proposition 9.7 and Lemma 9.8 directly imply the following. 
Corollary 9.9. We have $a_{j}^{\mu}=a_{j}^{\mathbf{R}_{i}(\mu)}$ for $j \neq i \bmod l$. Moreover $a_{l j+i}^{\mathbf{R}_{i}(\mu)}=a_{l j+i+1}^{\mu}+$ $a_{l j+i-1}^{\mu}-a_{l j+i}^{\mu}$ if $j \neq 0$ or $i \neq 0$ and $a_{0}^{\mathbf{R}_{0}(\mu)}=a_{1}^{\mu}+a_{-1}^{\mu}-a_{0}^{\mu}+1$ otherwise.

9.5. Removable and addable cells. Throughout this subsection let $\mu$ be an arbitrary partition. Recall the partition $\mathbf{T}_{k}(\mu)$ from Definition 4.9 obtained from $\mu$ by adding all $k$-addable cells and removing all $k$-removable cells. We will now interpret addability and removability in terms of the residue of $\mu$.

Lemma 9.10. Let us write $\operatorname{Res}_{\mu}(t)=\sum_{j \in \mathbb{Z}} b_{j} t^{j}$. Let $k \in \mathbb{Z}$.

a) The following are equivalent: (1) a cell of content $k$ is removable; (2) $b_{k-1}=b_{k}, b_{k+1}=$ $b_{k}-1(k>0)$ or $b_{k+1}=b_{k}, b_{k-1}=b_{k}-1(k<0)$ or $b_{-1}=b_{1}, b_{0}=b_{1}+1(k=0)$.

b) The following are equivalent: (1) a cell of content $k$ is addable; (2) $b_{k+1}=b_{k}, b_{k-1}=$ $b_{k}+1(k>0)$ or $b_{k-1}=b_{k}, b_{k+1}=b_{k}+1(k<0)$ or $b_{-1}=b_{0}=b_{1}(k=0)$.

c) The following are equivalent: (1) no cell of content $k$ is addable or removable; (2) $\left[b_{k+1}=b_{k}=b_{k-1}\right.$ or $\left.b_{k+1}=b_{k}-1=b_{k-1}-2(k>0)\right]$, or $\left[b_{k+1}=b_{k}=b_{k-1}\right.$ or $\left.b_{k+1}=b_{k}+1=b_{k-1}+2(k<0)\right]$, or $\left[b_{-1}=b_{0}=b_{1}+1\right.$ or $\left.b_{1}=b_{0}=b_{-1}+1(k=0)\right]$.

Proof. Let $k>0$. Suppose that the cell $(i, j)$ is removable from $\mathbb{Y}(\mu)$ and has content $k$. Then $j-i=k$ and $(1, k+1),(2, k+2), \ldots,(i, j)$ are precisely the cells of content $k$ in $\mathbb{Y}(\mu)$. Since $\mathbb{Y}(\mu)$ has a corner at $k$, the cells of content $k-1$ in $\mathbb{Y}(\mu)$ are precisely $(1, k),(2, k+1), \ldots,(i, j-1)$ and the cells of content $k+1$ in $\mathbb{Y}(\mu)$ are precisely $(1, k+$ $2),(2, k+3), \ldots,(i-1, j)$. Hence $b_{k}=i, b_{k-1}=i, b_{k+1}=i-1$, which yields the desired equalities. Conversely, suppose that $b_{k-1}=b_{k}, b_{k+1}=b_{k}-1$. Then the cell $\left(b_{k}, b_{k}+k\right)$ is removable. Indeed, $b_{k-1}=b_{k}$ implies that $\left(b_{k}+1, b_{k}+k\right) \notin \mathbb{Y}(\mu)$ and $b_{k+1}=b_{k}-1$ implies that $\left(b_{k}, b_{k}+k+1\right) \notin \mathbb{Y}(\mu)$. The proofs of the remaining cases are analogous.

9.6. Combinatorial interpretation of reflection functors. We can now interpret the effect of applying reflection functors to the fixed points combinatorially.

Theorem 9.11. Let $\mu \in \mathcal{P}_{\nu^{t}}\left(n l+\left|\nu^{t}\right|\right)$ and $0 \leq k \leq l-1$. We have $\mathbf{R}_{k}(\mu)=\left(\mathbf{T}_{k}\left(\mu^{t}\right)\right)^{t}$.

Proof. It suffices to show that the residue of $\mathbf{T}_{k}\left(\mu^{t}\right)$ equals $P_{\mathbf{R}_{k}(\mu)}$. Let us write

$$
\operatorname{Res}_{\mathbf{T}_{k}\left(\mu^{t}\right)}(t)=: \sum_{i \in \mathbb{Z}} a_{i} t^{i}, \quad \operatorname{Res}_{\mu^{t}}(t)=: \sum_{i \in \mathbb{Z}} b_{i} t^{i}, \quad P_{\mathbf{R}_{k}(\mu)}=: \sum_{i \in \mathbb{Z}} c_{i} t^{i}, \quad P_{\mu}=: \sum_{i \in \mathbb{Z}} d_{i} t^{i} .
$$

By Lemma 9.3, we have $b_{i}=d_{i}$. Suppose that $i \neq k \bmod l$. Then $a_{i}=b_{i}=d_{i}=c_{i}$. The first equality follows from the fact that no cells of content $i$ are added to or removed from $\mu^{t}$ when we transform $\mu^{t}$ into $\mathbf{T}_{k}\left(\mu^{t}\right)$. The third equality follows from Corollary 9.9

Now suppose that $i=k \bmod l$ and $i>0$. Then $c_{i}=d_{i+1}+d_{i-1}-d_{i}$ by Corollary 9.9. Hence $c_{i}=b_{i+1}+b_{i-1}-b_{i}$. We now argue that $b_{i+1}+b_{i-1}-b_{i}=a_{i}$. There are three possibilities: $a_{i}=b_{i}+1$ and one cell of content $i$ is addable to $\mu^{t}$, or $a_{i}=b_{i}-1$ and one cell of content $i$ is removable from $\mu^{t}$, or $a_{i}=b_{i}$ and no cell of content $i$ is addable to or removable from $\mu^{t}$. In the first case we have $b_{i}=b_{i+1}$ and $b_{i-1}=b_{i}+1$. In the second case we have $b_{i}=b_{i-1}$ and $b_{i+1}=b_{i}-1$. In the third case we have $b_{i-1}=b_{i}=b_{i+1}$ or $b_{i}=b_{i+1}+1=b_{i-1}-1$. These equalities follow immediately from Lemma 9.10, In each of the three cases we see that the equality $b_{i+1}+b_{i-1}-b_{i}=a_{i}$ holds. Hence $a_{i}=c_{i}$. The proof for $i \leq 0$ is analogous.

Recall that if $\lambda \in \mathcal{P}$ then

$$
\underline{\operatorname{Quot}}\left(\lambda^{t}\right)=\left(\underline{\mathrm{Quot}}(\lambda)^{t}\right)^{b} .
$$

Corollary 9.12. Let $\mu \in \mathcal{P}_{\nu^{t}}\left(n l+\left|\nu^{t}\right|\right)$ and $i \in\{0, \ldots, l-1\}$. Then $\mathbf{R}_{i}(\mu)=\left(\sigma_{i} * \mu^{t}\right)^{t}$. Moreover,

$$
\operatorname{Core}\left(\mathbf{R}_{i}(\mu)\right)=\left(\sigma_{i} * \nu\right)^{t}=\left(\mathbf{T}_{i}(\nu)\right)^{t}, \quad \underline{\operatorname{Quot}}\left(\mathbf{R}_{i}(\mu)\right)=s_{l-i} \cdot \underline{\operatorname{Quot}}(\mu) .
$$

Proof. The first claim follows directly from Theorem 9.11 and the definition of the $\tilde{S}_{l^{-}}$ action on partitions in $\$ 4.7$. The formula for $\operatorname{Core}\left(\mathbf{R}_{i}(\mu)\right)$ follows directly from Proposition 
4.10. The formula for $\operatorname{Quot}\left(\mathbf{R}_{i}(\mu)\right)$ follows from Proposition 4.10 and (49). Indeed,

$$
\begin{aligned}
\underline{\operatorname{Quot}}\left(\mathbf{R}_{i}(\mu)\right)=\underline{\operatorname{Quot}}\left(\left(\sigma_{i} * \mu^{t}\right)^{t}\right) & =\left(\left(\underline{\operatorname{Quot}}\left(\sigma_{i} * \mu^{t}\right)\right)^{t}\right)^{b} \\
& =\left(\left(s_{i} \cdot \underline{\operatorname{Quot}}\left(\mu^{t}\right)\right)^{t}\right)^{b} \\
& \left.=\left(s_{i} \cdot \underline{\underline{\operatorname{Quot}}}(\mu)\right)^{b}\right)^{b}=s_{l-i} \cdot \underline{\operatorname{Quot}}(\mu) .
\end{aligned}
$$

Note that, by Proposition 4.10, we also have:

$$
\operatorname{Core}\left(\left(\mathbf{R}_{i}(\mu)\right)^{t}\right)=\sigma_{i} * \nu=\mathbf{T}_{i}(\nu), \quad \underline{\operatorname{Quot}}\left(\left(\mathbf{R}_{i}(\mu)\right)^{t}\right)=\operatorname{pr}\left(\sigma_{i}\right) \cdot \underline{\text { Quot }}(\mu)=s_{i} \cdot \underline{\operatorname{Quot}}(\mu) .
$$

\section{Connection to the Hilbert scheme}

10.1. The Hilbert scheme. Let $K$ be a positive integer. We let $\mathrm{Hil}_{K}$ denote the Hilbert scheme of $K$ points in $\mathbb{C}^{2}$. The underlying set of the scheme $\mathrm{Hil}_{K}$ consists of ideals of $\mathbb{C}\left[z_{1}, z_{2}\right]$ of colength $K$, i.e., ideals $I \subset \mathbb{C}\left[z_{1}, z_{2}\right]$ such that $\operatorname{dim} \mathbb{C}\left[z_{1}, z_{2}\right] / I=K$.

We let $\mathbb{C}^{*}$ act on $\mathbb{C}\left[z_{1}, z_{2}\right]$ by the rule $t . z_{1}=t z_{1}, t . z_{2}=t^{-1} z_{2}$. This action induces an action on $\mathrm{Hil}_{K}$. The $\mathbb{C}^{*}$-fixed points in $\mathrm{Hil}_{K}$ are precisely the monomial ideals in $\mathbb{C}\left[z_{1}, z_{2}\right]$. Let $\lambda \in \mathcal{P}(K)$. Let $I_{\lambda}$ be the $\mathbb{C}$-span of the monomials $\left\{z_{1}^{i} z_{2}^{j} \mid(i+1, j+1) \notin \mathbb{Y}(\lambda)\right\}$. We have a bijection

$$
\mathcal{P}(K) \longleftrightarrow \mathrm{Hil}_{K}^{\mathbb{C}^{*}}, \quad \lambda \mapsto I_{\lambda} .
$$

Let $\mathcal{T}_{K}$ denote the tautological bundle on $\mathrm{Hil}_{K}$. Its fibre $\left(\mathcal{T}_{K}\right)_{I}$ at $I$ is isomorphic to $\mathbb{C}\left[z_{1}, z_{2}\right] / I$. The following lemma follows immediately from the definitions.

Lemma 10.1. Let $\lambda \in \mathcal{P}(K)$. We have $\operatorname{ch}_{t}\left(\mathcal{T}_{K}\right)_{I_{\lambda}}=\operatorname{Res}_{\lambda^{t}}(t)$.

There is also a $\mathbb{Z} / l \mathbb{Z}$-action on $\mathrm{Hil}_{K}$ induced by the $\mathbb{Z} / l \mathbb{Z}$-action on $\mathbb{C}\left[z_{1}, z_{2}\right]$ given by $\epsilon . z_{1}=\eta^{-1} z_{1}, \epsilon . z_{2}=\eta z_{2}$.

10.2. Connection to rational Cherednik algebras. Set $-\mathbf{1}:=-\frac{1}{l}(1, \ldots, 1) \in \mathbb{Q}^{l}$ and $-\frac{1}{2}:=-\frac{1}{2 l}(1, \ldots, 1) \in \mathbb{Q}^{l}$. Let $w \in \tilde{S}_{l}$ and set $\theta:=w^{-1} \cdot\left(-\frac{1}{2}\right) \in \mathbb{Q}^{l}$ as well as $\gamma:=w * n \delta \in \mathbb{Z}^{l}$. We have $\gamma=n \delta+\gamma_{0}$, where $\gamma_{0}=w * \varnothing$. Let $\nu:=\mathfrak{d}^{-1}\left(\gamma_{0}\right)$ be the $l$-core corresponding to $\gamma_{0}$. By [16, Lemmas $\left.4.3,7.2\right]$, the quiver variety $\mathcal{X}_{\theta}(n \delta)$ is smooth. Set $\mathbf{h}:=\left(h, H_{1}, \ldots, H_{l-1}\right)$ with $H_{j}=\theta_{j}(1 \leq j \leq l-1)$ and $h=-\theta_{0}-\sum_{j=1}^{l-1} H_{j}$. Let us fix a reduced expression $w=\sigma_{i_{1}} \cdots \sigma_{i_{m}}$ for $w$ in $\tilde{S}_{l}$. Composing reflection functors yields a $U(1)$-equivariant hyper-Kähler isometry

$$
\mathfrak{R}_{i_{1}} \circ \cdots \circ \mathfrak{R}_{i_{m}}: \mathcal{X}_{\theta}(n \delta) \stackrel{\sim}{\rightarrow} \mathcal{X}_{-\frac{1}{2}}(\gamma) .
$$

By [16, §3.7] there exists a $U(1)$-equivariant diffeomorphism

$$
\mathcal{X}_{-\frac{1}{2}}(\gamma) \stackrel{\sim}{\rightarrow} \mathcal{M}_{-1}(\gamma) .
$$

Set $K=n l+|\nu|$. By [16, Lemma 7.8], there is a $\mathbb{C}^{*}$-equivariant embedding

$$
\mathcal{M}_{-\mathbf{1}}(\gamma) \hookrightarrow \mathrm{Hil}_{K} .
$$

Its image is the component $\mathrm{Hil}_{K}^{\nu}$ of $\mathrm{Hil}_{K}^{\mathbb{Z} / l \mathbb{Z}}$ whose generic points have the form $V\left(I_{\nu}\right) \cup O$, where $O$ is a union of $n$ distinct free $\mathbb{Z} / l \mathbb{Z}$-orbits in $\mathbb{C}^{2}$. Moreover, $\left(\operatorname{Hil}_{K}^{\nu}\right)^{\mathbb{C}^{*}}=\left\{I_{\lambda} \mid \lambda \in\right.$ $\left.\mathcal{P}_{\nu}(n l+|\nu|)\right\}$. Let

$$
\Phi: \mathcal{X}_{-\frac{1}{2}}(\gamma) \rightarrow \mathcal{M}_{-1}(\gamma) \rightarrow \operatorname{Hil}_{K}^{\nu}
$$

be the composition of $(52)$ and $(53)$. It induces a bijection between the $\mathbb{C}^{*}$-fixed points and hence also a bijection between their labelling sets

$$
\Psi: \mathcal{P}_{\nu^{t}}\left(n l+\left|\nu^{t}\right|\right) \rightarrow \mathcal{P}_{\nu}(n l+|\nu|), \quad \mu \mapsto \lambda,
$$

where the partition $\lambda$ is defined by the equation $I_{\lambda}=\Phi([\mathbf{A}(\mu)])$.

Lemma 10.2. Let $\mu \in \mathcal{P}_{\nu^{t}}\left(n l+\left|\nu^{t}\right|\right)$. We have $\Psi(\mu)=\mu^{t}$.

Proof. Let $\mathcal{V}_{-\frac{1}{2}}(\gamma):=\mu_{\gamma}^{-1}\left(-\frac{1}{2}\right) \times{ }^{G(\gamma)} \widehat{\mathbf{V}}^{\nu}$ denote the tautological bundle on $\mathcal{X}_{-\frac{1}{2}}(\gamma)$. The diffeomorphism 54 lifts to a $U(1)$-equivariant isomorphism of tautological vector bundles

$$
\mathcal{V}_{-\frac{1}{2}}(\gamma) \stackrel{\cong}{\rightarrow} \mathcal{T}_{K}^{\nu},
$$

where $\mathcal{T}_{K}^{\nu}$ denotes the restriction of $\mathcal{T}_{K}$ to the subscheme $\mathrm{Hil}_{K}^{\nu}$. Proposition 5.15 implies that $\operatorname{ch}_{t} \mathcal{V}_{-\frac{1}{2}}(\gamma)_{[\mathbf{A}(\mu)]}=\operatorname{Res}_{\mu}(t)$. Hence the $\mathbb{C}^{*}$-characters of the fibres of $\mathcal{V}_{-\frac{1}{2}}(\gamma)$ at any 
two distinct $\mathbb{C}^{*}$-fixed points are distinct. By Lemma 10.1 we have $\operatorname{ch}_{t}\left(\mathcal{T}_{K}^{\nu}\right)_{I_{\mu}}=\operatorname{Res}_{\mu^{t}}(t)$. The $U(1)$-equivariance of (55) implies that $\Phi([\mathbf{A}(\mu)])=I_{\mu^{t}}$ and so $\Psi(\mu)=\mu^{t}$.

We are now ready to collect all our results about the $\mathbb{C}^{*}$-fixed points. We have a sequence of equivariant isomorphisms (of varieties or manifolds):

$$
\mathcal{Y}_{\mathbf{h}} \stackrel{\mathrm{EG}}{\longrightarrow} \mathcal{X}_{\theta}(n \delta) \stackrel{\Re_{i_{1}} \circ \cdots \circ \Re_{i_{m}}}{\longrightarrow} \mathcal{X}_{-\frac{1}{2}}(\gamma) \stackrel{\Phi}{\rightarrow} \mathrm{Hil}_{K}^{\nu}
$$

They induce bijections between the labelling sets of $\mathbb{C}^{*}$-fixed points.

Theorem 10.3. The map (56) induces the following bijections

$$
\begin{aligned}
& \mathcal{P}(l, n) \longrightarrow \mathcal{P}_{\varnothing}(n l) \longrightarrow \mathcal{P}_{\nu^{t}}\left(n l+\left|\nu^{t}\right|\right) \longrightarrow \mathcal{P}_{\nu}(n l+|\nu|) \\
& \underline{\operatorname{Quot}}(\mu)^{b} \longmapsto \mu \quad \longmapsto \quad\left(w * \mu^{t}\right)^{t} \quad \longmapsto \quad w * \mu^{t} .
\end{aligned}
$$

Moreover,

$$
\nu=w * \varnothing=\mathbf{T}_{i_{1}} \circ \ldots \circ \mathbf{T}_{i_{m}}(\varnothing), \quad \underline{\operatorname{Quot}}\left(w * \mu^{t}\right)=\operatorname{pr}(w) \cdot \underline{\operatorname{Quot}}\left(\mu^{t}\right) .
$$

Proof. The theorem just collects the results of Theorem 7.18, Corollary 9.12 , Lemma 10.2 and 50 .

Let us rephrase our result slightly. Given $w \in \tilde{S}_{l}$, we define the $w$-twisted l-quotient bijection to be the map

$$
\tau_{w}: \mathcal{P}(l, n) \rightarrow \mathcal{P}_{\nu}(n l+|\nu|), \quad \underline{\operatorname{Quot}}(\mu) \mapsto w * \mu .
$$

Corollary 10.4. The bijection $\mathcal{P}(l, n) \rightarrow \mathcal{P}_{\nu}(n l+|\nu|)$ induced by 56 is given by

$$
\underline{\lambda} \mapsto \tau_{w}\left(\underline{\lambda}^{t}\right) .
$$

Proof. Suppose that $\underline{\lambda}=\underline{Q}$ uot $(\mu)^{b}$. Then Theorem 10.3 implies that $\underline{\lambda}$ is sent to $w * \mu^{t}$. On the other hand, $\left.\underline{\lambda}^{\bar{t}}=\underline{(\underline{\mathrm{Quot}}}(\mu)^{b}\right)^{t}=\underline{\mathrm{Quot}}\left(\mu^{t}\right)$ by $(49)$. Hence $\tau_{w}\left(\underline{\lambda}^{\underline{t}}\right)=w * \mu^{t}$.

Remark 10.5. The statement of Corollary 10.4 appears in the proof of 16, Proposition 7.10]. However, the proof of this statement in [16] is incorrect. The problem lies in an incorrect assumption about the function $c_{\mathbf{h}}: \mathcal{P}(l, n) \times \mathbb{Q}^{l} \rightarrow \mathbb{Q}$, defined by:

$$
c_{\mathbf{h}}(\underline{\lambda})=l \sum_{i=1}^{l-1}\left|\lambda^{i}\right|\left(H_{1}+\ldots+H_{i}\right)-l\left(\frac{n(n-1)}{2}+\sum_{i=0}^{l-1} n\left(\lambda^{i}\right)-n\left(\left(\lambda^{i}\right)^{t}\right)\right) h .
$$

Given $\mathbf{h} \in \mathbb{Q}^{l}$, the function $c_{\mathbf{h}}$ induces an ordering on $\mathcal{P}(l, n)$, called the $c$-order, given by the rule

$$
\underline{\mu}<_{\mathbf{h}} \underline{\lambda} \Longleftrightarrow c_{\mathbf{h}}(\underline{\mu})<c_{\mathbf{h}}(\underline{\lambda}) .
$$

Dependence of this order on $\mathbf{h}$ decomposes the parameter space $\mathbb{Q}^{l}$ into a finite number of so-called $c$-chambers. It is stated in [16, §2.5] that the $c$-order is a total order inside $c$-chambers. This, however, is not true. Let us consider counterexamples in which the $c$-order is not total for all values of $\mathbf{h}$. For example, take $l=1$. Then

$$
c_{\mathbf{h}}(\lambda)=-\left(\frac{n(n-1)}{2}+n(\lambda)-n\left(\lambda^{t}\right)\right) h .
$$

It follows immediately from (58) that $c_{\mathbf{h}}(\lambda)=c_{\mathbf{h}}(\mu)$ for all values of $\mathbf{h}$ if $\lambda$ and $\mu$ are two symmetric partitions in $\mathcal{P}(n)$. There are other examples. Take $\mu=(6,3,2,2,2)$. Then $n(\mu)=3+4+6+8=21$. Since $\mu^{t}=(5,5,2,1,1,1)$ we have $n\left(\mu^{t}\right)=5+4+3+4+5=21$. It follows that $\mu$ and $\mu^{t}$ are incomparable in the $c$-order for all values of $\mathbf{h}$.

Gordon's proof of (57) relies on comparing the values of some Morse functions on the quiver variety $\mathcal{M}_{2 \theta}(n \delta)$ and the Hilbert scheme at the $\mathbb{C}^{*}$-fixed points. This approach would work if the Morse functions assigned distinct values to each fixed point. However, this isn't the case because the Morse function on $\mathcal{M}_{2 \theta}(n \delta)$, evaluated at the fixed points, is given by $c_{\mathbf{h}}$. 
10.3. The combinatorial and geometric orderings. In [16, §5.4] Gordon defines a geometric ordering $\preceq_{w}^{\text {geo }}$ on $\mathcal{P}(l, n)$ using the closure relations between the attracting sets of $\mathbb{C}^{*}$-fixed points in $\mathcal{M}_{2 \theta}(n \delta)$ (Gordon uses the notation $\preceq_{\mathbf{h}}$ ). We also have a combinatorial ordering on $\mathcal{P}(l, n)$ given by:

$$
\underline{\mu} \preceq_{w}^{\text {com }} \underline{\lambda} \Longleftrightarrow \tau_{w}\left(\underline{\lambda}^{t}\right) \unlhd \tau_{w}\left(\underline{\mu}^{t}\right),
$$

where $\unlhd$ denotes the dominance ordering on partitions.

Corollary 10.6. Let $w \in \tilde{S}_{l}$ and $\underline{\mu}, \underline{\lambda} \in \mathcal{P}(l, n)$. Then $\underline{\mu} \underline{w}_{w}^{\text {geo }} \underline{\lambda} \Rightarrow \underline{\mu} \preceq_{w}^{\text {com }} \underline{\lambda}$.

Proof. Using the closure relations between the attracting sets of $\mathbb{C}^{*}$-fixed points in $\mathrm{Hil}_{K}^{\nu}$, one can also define a geometric ordering $\preceq^{\text {geo }}$ on $\mathcal{P}_{\nu}(n l+|\nu|)$. By construction, the isomorphism $\mathcal{M}_{2 \theta}(n \delta) \stackrel{\sim}{\longrightarrow} \mathrm{Hil}_{K}^{\nu}$ intertwines the two geometric orderings. Hence, by Corollary 10.4 $\underline{\mu} \preceq_{w}^{\text {geo }} \underline{\lambda} \Longleftrightarrow \tau_{w}\left(\underline{\mu}^{t}\right) \preceq$ geo $\tau_{w}\left(\underline{\lambda}^{t}\right)$. But, by [29, (4.13)], the ordering $\preceq$ geo is refined by the anti-dominance ordering on $\mathcal{P}_{\nu}(n l+|\nu|)$.

\section{REFERENCES}

[1] Bellamy G., On singular Calogero-Moser spaces, Bull. Lon. Math. Soc. 41, No. 2 (2009), 315-326.

[2] Bellamy G., Rational Cherednik algebras and Schubert cells, arXiv:1210.3870

[3] Bonnafé C., Maksimau R., Fixed points in smooth Calogero-Moser spaces, arXiv:1803.04287

[4] Bezrukavnikov R., Finkelberg M., Wreath Macdonald polynomials and categorical McKay correspondence, Camb. J. Math. 2, No. 2 (2014), 163-190.

[5] Cherednik I., Double affine Hecke algebras, Knizhnik-Zamolodchikov equations, and Macdonald operators, IMRN (Duke math. J.) 9 (1992), 171-180.

[6] Chen X., Stanley R.P., A formula for the specialization of skew Schur functions, Ann. Comb. (2016), $1-10$.

[7] Crawley-Boevey W., Holland M.P., Geometry of the moment map for representations of quivers Com. Math. (2001), 257-293.

[8] Crawley-Boevey W., Holland M.P., Noncommutative deformations of Kleinian singularities Duke Math. 92 (1998), 605-635.

[9] Dezélée C., Generalized graded Hecke algebra for complex reflection group of type $G(r, 1, n)$, Preprint 2006, arXiv:0605410v2.

[10] Dunkl C., Griffeth S., Generalized Jack polynomials and the representation theory of rational Cherednik algebras, Sel. Math. 16, No. 4 (2010), 791-818.

[11] Etingof P., Ma X., Lecture notes on Cherednik algebras, arXiv:1001.0432

[12] Etingof P., Ginzburg V., Symplectic reflection algebras, Calogero-Moser spaces, and deformed Harish-Chandra homomorphism, Inv. Math. 147 (2002), 243-348.

[13] Frenkel I., Savage A., Bases of representations of type A affine Lie algebras via quiver varieties and statistical mechanics, Int. Math. Res. Not., No. 28 (2003), 1521-1547.

[14] Garsia A.M., Haiman M., A random $(q, t)$-walk and a sum of Pieri coefficients, J. Combin. Theory, Ser. A 82 (1998), 74-111.

[15] Gordon I., Baby Verma modules for rational Cherednik algebras, Bull. London Math. Soc. 35 (2003), 321-336.

[16] Gordon I., Quiver varieties, category $\mathcal{O}$ for rational Cherednik algebras, and Hecke algebras, Int. Math. Res. Pap. (2008).

[17] Gordon I., Martino M., Calogero-Moser space, restricted rational Cherednik algebras and two-sided cells, Math. Res. Lett. 16, No. 2-3 (2009), 255-262.

[18] Guay N., Quantum algebras and symplectic reflection algebras for wreath products, Rep. Th. 14 (2010), 148-200.

[19] Haiman M., Hilbert schemes, polygraphs and the Macdonald positivity conjecture, J. Amer. Math. Soc. 14, No.4 (2001).

[20] Haiman M., Combinatorics, symmetric functions, and Hilbert schemes. Current developments in mathematics, Int. Press, Somerville, MA, 2003.

[21] James G., Kerber A., The representation theory of the symmetric group, Addison-Wesley Publishing Company, Reading, MA, 1981.

[22] Kerov S., A q-analog of the hook walk algorithm and random Young tableaux, Func. Anal. App. 26, No. 3 (1992), 179-187.

[23] Lam T., Lapointe L., Morse J., Shimozono M., Affine insertion and Pieri rules for the affine Grassmannian, Am. J. of Math. 128, No. 6 (2006), 1553-1586.

[24] Lam T., Affine Stanley symmetric functions, Memoirs of the AMS, 2010.

[25] Van Leeuwen M., Edge sequences, ribbon tableaux, and an action of affine permutations, Europ. J. of Comb. 20 (1999), 179-195.

[26] Losev I., Procesi bundles and symplectic reflection algebras, arXiv:1501.00643

[27] Maffei A., A remark on quiver varieties and Weyl groups, Ann. Sc. Norm. Sup. Pisa - Cl. Sc. 5 (2002), No. 3, 649-686.

[28] Martino M., Blocks of restricted rational Cherednik algebras for $G(m, d, n)$, J. of Alg. 397 (2014), 209-224. 
[29] Nakajima H., Jack polynomials and Hilbert schemes of points on surfaces, math.AG/9610021, 1996.

[30] Nakajima H., Reflection functors for quiver varieties and Weyl group actions, Math. Ann. 327, No. 4 (2003), 671-721.

[31] Pushkarev I.A., On the representation theory of wreath products of finite groups and symmetric groups, J. of Math. Sci. 69, No. 5 (1999), 3590-3599.

[32] Ram A., Shepler A., Classification of graded Hecke algebras for complex reflection groups, Comment. Math. Helv. 78 (2003), 308-334.

[33] Rouquier R., q-Schur algebras and complex reflection groups, Mosc. Math. J. 8, No. 1 (2003), $119-158$.

[34] Savage A., A geometric boson-fermion correspondence, C.R. Math. Acad. Sci. Soc. R. Can. 28, No. 3 (2006), 65-84.

[35] Stembridge J.R., On the eigenvalues of representations of reflection groups and wreath products, Pac. J. of Math. 140, No. 2 (1989), 353-396.

[36] Wilson G., Collisions of Calogero-Moser particles and an adelic Grassmannian, Inv. Math. 133 (1998), 1-44. 